\title{
Total Synthesis of the Illicium-Derived Sesquineolignan Simonsol C
}

Jeremy Nugent, Martin G. Banwell,* and Brett D. Schwartz

Research School of Chemistry, Institute of Advanced Studies, The Australian National University, Canberra, ACT 2601, Australia

\section{CONTENTS}

General Experimental Protocols

Specific Chemical Transformations

Table S1: Comparison of the ${ }^{13} \mathrm{C}$ NMR Data Recorded on Compound $( \pm)-\mathbf{1}$ with Literature Values

X-ray Crystallographic Data for Compound 27

Anisotropic Displacement Ellipsoid Plot from the Single-crystal X-ray

Analysis of Compound $\mathbf{2 7}$

References

${ }^{1} \mathrm{H}$ and ${ }^{13} \mathrm{C}$ NMR Spectra of Compounds 8, 10, 11, 15-19, 12, 21-23, 1, 26-30

and $( \pm)-\mathbf{1}$

\section{PAGE}

S2

S3

S24

S 25

S26

S27

S28 


\section{General Experimental Protocols}

Unless otherwise specified, proton $\left({ }^{1} \mathrm{H}\right)$ and carbon $\left({ }^{13} \mathrm{C}\right)$ NMR spectra were recorded at room temperature in base-filtered $\mathrm{CDCl}_{3}$ on a spectrometer operating at $400 \mathrm{MHz}$ for proton and $100 \mathrm{MHz}$ for carbon nuclei. For ${ }^{1} \mathrm{H}$ NMR spectra, signals arising from the residual protioforms of the solvent were used as internal standards. ${ }^{1} \mathrm{H}$ NMR data are recorded as follows: chemical shift ( $\delta$ ) [multiplicity, coupling constant(s) $J(\mathrm{~Hz})$, relative integral] where multiplicity is defined as: $\mathrm{s}=$ singlet; $\mathrm{d}=$ doublet; $\mathrm{t}=$ triplet; $\mathrm{q}=$ quartet; $\mathrm{m}=$ multiplet or combinations of the above. The signal due to residual $\mathrm{CHCl}_{3}$ appearing at $\delta_{\mathrm{H}} 7.26$ and the central resonance of the $\mathrm{CDCl}_{3}$ "triplet" appearing at $\delta_{\mathrm{C}} 77.0$ were used to reference ${ }^{1} \mathrm{H}$ and ${ }^{13} \mathrm{C}$ NMR spectra, respectively. Infrared spectra $\left(\mathrm{n}_{\max }\right)$ were recorded on a FTIR Spectrometer. Samples were analyzed as thin films on $\mathrm{KBr}$ plates. Low-resolution ESI mass spectra were recorded on a single quadrupole liquid chromatograph-mass spectrometer, while high-resolution measurements were conducted on a time-of-flight instrument. Low- and high-resolution EI mass spectra were recorded on a magnetic-sector machine. Melting points were measured on an automated melting point system and are uncorrected. Analytical thin layer chromatography (TLC) was performed on aluminum-backed $0.2 \mathrm{~mm}$ thick silica gel $60 \mathrm{~F}_{254}$ plates. Eluted plates were visualized using a $254 \mathrm{~nm}$ UV lamp and/or by treatment with a suitable dip followed by heating. These dips included phosphomolybdic acid : ceric sulfate : sulfuric acid (conc.) : water $(37.5 \mathrm{~g}: 7.5 \mathrm{~g}: 37.5 \mathrm{~g}: 720 \mathrm{~mL})$ or potassium permanganate : potassium carbonate : $5 \%$ sodium hydroxide aqueous solution : water $(3 \mathrm{~g}: 20 \mathrm{~g}: 5 \mathrm{~mL}: 300 \mathrm{~mL})$. Flash chromatographic separations were carried out following protocols defined by Still et al. ${ }^{1}$ with silica gel $60(40-63 \mathrm{~mm})$ as the stationary phase and using the AR- or HPLC-grade solvents indicated. Starting materials, reagents and drying agents as well as other inorganic salts were generally available from commercial sources and used as supplied. Tetrahydrofuran (THF), diethyl ether, methanol and dichloromethane were dried using a solvent purification system that is based upon a technology originally described by Grubbs et $a l^{2}$ Where necessary, reactions were performed under an nitrogen atmosphere. 


\section{Specific Chemical Transformations}

Compound 8

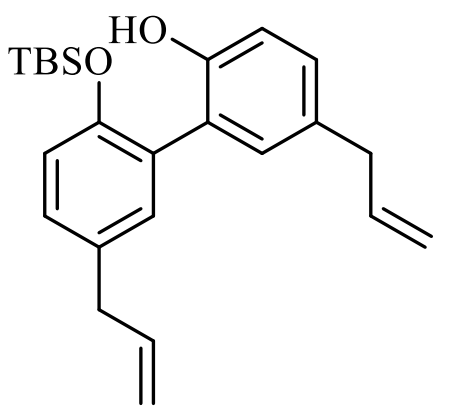

8

A magnetically stirred solution of magnolol (7) (1.00 g, $3.76 \mathrm{mmol})$ and imidazole $(272 \mathrm{mg}, 4.00 \mathrm{mmol})$ in dry dichloromethane (40 mL) maintained at $22{ }^{\circ} \mathrm{C}$ under a nitrogen atmosphere was treated with TBS-Cl (301 mg, $\left.5.00 \mathrm{mmol}\right)$. The ensuing light-yellow mixture was stirred at this temperature for $1 \mathrm{~h}$ then treated with $\mathrm{NH}_{4} \mathrm{Cl}(30 \mathrm{~mL}$ of a saturated aqueous solution) before being extracted with dichloromethane $(3 \mathrm{x} 20$ $\mathrm{mL})$. The combined organic phases were washed with brine $(1 \times 50 \mathrm{~mL})$ then dried $\left(\mathrm{Na}_{2} \mathrm{SO}_{4}\right)$, filtered, and concentrated under reduced pressure. The residue thus obtained was subjected to flash chromatography (silica, 9:1 v/v hexane/ethyl acetate elution) to afford, after concentration of the appropriate fractions $\left(R_{f}=0.6\right.$ in $95: 5 \mathrm{v} / \mathrm{v}$ hexane/ethyl acetate $)$, compound $\mathbf{8}(1.40 \mathrm{~g}, 98 \%)$ as a light-yellow oil.

${ }^{1} \mathbf{H}$ NMR $\left(400 \mathrm{MHz}, \mathrm{CDCl}_{3}\right) \delta 7.13(\mathrm{~d}, J=2.3 \mathrm{~Hz}, 1 \mathrm{H}), 7.09(\mathrm{~m}, 2 \mathrm{H}), 7.05(\mathrm{~d}, J=2.3 \mathrm{~Hz}, 1 \mathrm{H}), 6.93(\mathrm{~d}, J=8.2 \mathrm{~Hz}, 1 \mathrm{H}), 6.89(\mathrm{~d}, J=8.2 \mathrm{~Hz}$, $1 \mathrm{H}), 6.41(\mathrm{~s}, 1 \mathrm{H}), 5.98(\mathrm{~m}, 2 \mathrm{H}), 5.15-5.05$ (complex m, 4H), $3.37(\mathrm{~m}, 4 \mathrm{H}), 0.83(\mathrm{~s}, 9 \mathrm{H}),-0.05$ (broad s, 6H)

${ }^{13}$ C NMR $\left(100 \mathrm{MHz}_{\mathrm{CDCl}}\right) \delta 152.2,150.0,138.2,137.6,134.8,132.4,132.3,131.2,130.2,129.5,129.1,127.1,120.7,117.9,116.0,115.4$, $39.6,39.5,25.6,18.1,-4.6$

IR (KBr) $v_{\max } 3397,2955,2931,2858,1639,1494,1257,1231,912,877,840 \mathrm{~cm}^{-1}$

MS (ESI, -ve) $m / z 379$ [(M - H) $\left.{ }^{-}, 90 \%\right], 265$ (100)

HRMS (EI, $70 \mathrm{eV}$ ) $\mathrm{M}^{+\bullet}$ calcd for $\mathrm{C}_{24} \mathrm{H}_{32} \mathrm{O}_{2} \mathrm{Si} 380.2172$, found 380.2177 . 


\section{Compound 9}

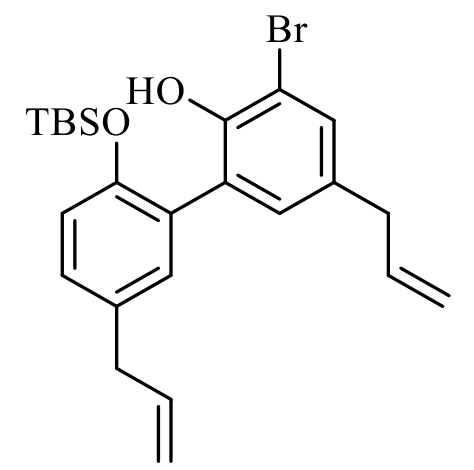

9

A magnetically stirred solution of compound $8(1.30 \mathrm{~g}, 3.42 \mathrm{mmol})$ in dry THF $(13 \mathrm{~mL})$ maintained at $-78{ }^{\circ} \mathrm{C}$ was treated with $i$-PrMgBr $(1.6$ $\mathrm{mL}$ of a $2.3 \mathrm{M}$ solution in 2-methyltetrahydrofuran, $3.70 \mathrm{mmol}$ ). The ensuing mixture was maintained at this temperature for $0.5 \mathrm{~h} \mathrm{before} \mathrm{being}$ treated with DBDMH $(772 \mathrm{mg}, 2.70 \mathrm{mmol})$. The resulting yellow mixture was maintained at $-78^{\circ} \mathrm{C}$ for $1 \mathrm{~h}$ before being treated with $\mathrm{NH}_{4} \mathrm{Cl}(30$ $\mathrm{mL}$ of a saturated aqueous solution) and then extracted with diethyl ether $(3 \times 30 \mathrm{~mL})$. The combined organic phases were washed with brine (1 x $50 \mathrm{~mL}$ ) then dried $\left(\mathrm{Na}_{2} \mathrm{SO}_{4}\right)$, filtered, and concentrated under reduced pressure. The residue thus obtained was subjected to flash chromatography (silica, 95:5 v/v hexane/ethyl acetate elution) to afford, after concentration of the appropriate fractions $\left(R_{\mathrm{f}}=0.7\right.$ in $95: 5 \mathrm{v} / \mathrm{v}$ hexane/ethyl acetate), compound $\mathbf{9}(1.40 \mathrm{~g}, 89 \%)$ as a light-yellow oil.

${ }^{1}$ H NMR $\left(400 \mathrm{MHz}, \mathrm{CDCl}_{3}\right) \delta 7.34(\mathrm{~d}, J=2.2 \mathrm{~Hz}, 1 \mathrm{H}), 7.11(\mathrm{~m}, 2 \mathrm{H}), 7.00(\mathrm{~d}, J=2.2 \mathrm{~Hz}, 1 \mathrm{H}), 6.87(\mathrm{~m}, 1 \mathrm{H}), 6.60(\mathrm{~s}, 1 \mathrm{H}), 5.89(\mathrm{~m}, 2 \mathrm{H}), 5.15-$ 5.05 (complex m, 4H), $3.38(\mathrm{~d}, J=6.8 \mathrm{~Hz}, 1 \mathrm{H}), 3.33(\mathrm{~d}, J=6.8 \mathrm{~Hz}, 2 \mathrm{H}), 0.82(\mathrm{~s}, 9 \mathrm{H}),-0.03$ (broadened s, 6H)

${ }^{13} \mathrm{C}$ NMR $\left(100 \mathrm{MHz}_{\mathrm{CDCl}}\right) \delta 150.2,148.7,137.5,137.3,134.5,133.5,132.4,132.0,130.8,129.6,129.5,128.3,120.6,116.2,116.1,112.0$, 39.6, 39.1, 25.5, 18.1, -4.6

IR (KBr) $v_{\max } 3521,3342,2955,2930,2858,1641,1494,1471,1248,916,841,783 \mathrm{~cm}^{-1}$

MS (ESI, -ve) $m / z 459$ and 457 [(M - H) $)^{-}, 50$ and 45\%], 345 and 343 (both 30), 81 and 79 (95 and 100)

HRMS (ESI, -ve) $m / z\left[(\mathrm{M}-\mathrm{H})^{-}\right.$calcd for $\mathrm{C}_{24} \mathrm{H}_{30}{ }^{79} \mathrm{BrO}_{2} \mathrm{Si} 457.1198$, found 457.1180 . 


\section{Compound 13}

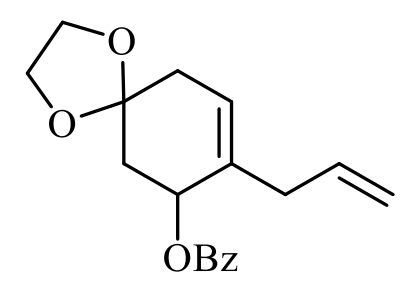

13

A magnetically stirred solution of compound $\mathbf{1 2}^{3}(7.91 \mathrm{~g}, 19.4 \mathrm{mmol})$ and $\mathrm{LiCl}(2.48 \mathrm{~g}, 58.5 \mathrm{mmol})$ in dry THF (130 mL) was treated, sequentially, with allyltributylstannane $(7.25 \mathrm{~mL}, 23.4 \mathrm{mmol})$ and $\mathrm{Pd}\left(\mathrm{PPh}_{3}\right)_{4}(2.25 \mathrm{~g}, 10 \mathrm{~mol} \%)$. The resulting yellow mixture was heated at reflux for $3 \mathrm{~h}$ then cooled and treated with $\mathrm{NH}_{4} \mathrm{Cl}(30 \mathrm{~mL}$ of a saturated aqueous solution) before being extracted with diethyl ether ( $3 \times 30 \mathrm{~mL})$. The combined organic phases were washed with brine $(1 \mathrm{x} 50 \mathrm{~mL})$ then dried $\left(\mathrm{Na}_{2} \mathrm{SO}_{4}\right)$, filtered, and concentrated under reduced pressure. The residue thus obtained was subjected to flash chromatography (silica, 8.5:1:0.5 v/v hexane/ethyl acetate/triethylamine elution) to afford, after concentration of the appropriate fractions $\left(R_{\mathrm{f}}=0.6\right.$ in $7: 3 \mathrm{v} / \mathrm{v}$ hexane/ethyl acetate), compound $13(3.79 \mathrm{~g}, 65 \%)$ as a light-yellow oil.

${ }^{1}$ H NMR $\left(400 \mathrm{MHz}, \mathrm{CDCl}_{3}\right) \delta 8.07(\mathrm{~m}, 2 \mathrm{H}), 7.56(\mathrm{~m}, 1 \mathrm{H}), 7.45(\mathrm{~m}, 2 \mathrm{H}), 5.88-5.71$ (complex m, 2H), 5.66 (m, 1H), 5.08-4.95 (complex m, $2 \mathrm{H}), 4.00(\mathrm{~m}, 4 \mathrm{H}), 2.86(\mathrm{~m}, 2 \mathrm{H}), 2.48(\mathrm{~m}, 1 \mathrm{H}), 2.40-2.27($ complex $\mathrm{m}, 2 \mathrm{H}), 2.00(\mathrm{dd}, J=12.8$ and $7.2 \mathrm{~Hz}, 1 \mathrm{H})$

${ }^{13} \mathbf{C}$ NMR $\left(100 \mathrm{MHz}_{2} \mathrm{CDCl}_{3}\right) \delta 166.3,135.5,135.0,133.1,130.5,129.8,128.5,124.1,117.0,107.6,71.5,64.7,64.6,37.4,37.2,36.1$

IR $(\mathrm{KBr}) v_{\max } 2971,2920,2879,1715,1265,1108,1069,1052,1026,948,712 \mathrm{~cm}^{-1}$

MS (ESI, +ve) $m / z 323\left[(\mathrm{M}+\mathrm{Na})^{+}, 100 \%\right]$

HRMS (ESI, +ve) $m / z(\mathrm{M}+\mathrm{Na})^{+}$calcd for $\mathrm{C}_{18} \mathrm{H}_{20} \mathrm{NaO}_{4} 323.1259$, found 323.1259. 


\section{Compound 5}

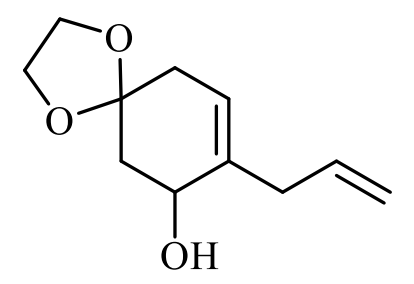

5

A magnetically stirred solution of compound $\mathbf{1 3}(1.00 \mathrm{~g}, 3.33 \mathrm{mmol})$ in methanol $(50 \mathrm{~mL})$ was treated with $\mathrm{NaOH}(10 \mathrm{~mL}$ of a $3 \mathrm{M}$ aqueous solution, $30 \mathrm{mmol})$ then heated at reflux for $2 \mathrm{~h}$. The resulting solution was cooled then treated with water (300 mL) and extracted with diethyl ether $(5 \times 60 \mathrm{~mL})$. The combined organic phases were washed with brine $(1 \times 50 \mathrm{~mL})$ before being dried $\left(\mathrm{Na}_{2} \mathrm{SO}_{4}\right)$, filtered, and concentrated under reduced pressure to give compound $5(607 \mathrm{mg}, 93 \%)$ as a clear, colorless oil. This material was used without further purification in the next step of the reaction sequence.

$\boldsymbol{R}_{\mathbf{f}}=0.4($ in $7: 3 \mathrm{v} / \mathrm{v}$ hexane/ethyl acetate)

${ }^{1}$ H NMR (400 MHz, $\left.\mathrm{CDCl}_{3}\right) 5.84(\mathrm{~m}, 1 \mathrm{H}), 5.45$ (broad s, 1H), 5.13-5.00 (complex m, 2H), 4.11 (m, 1H), 4.05-3.90 (complex m, 4H), 3.112.86 (complex m, 3H), $2.30(\mathrm{~m}, 2 \mathrm{H}), 2.08(\mathrm{dt}, J=13.8$ and $2.3 \mathrm{~Hz}, 1 \mathrm{H}), 1.98(\mathrm{dd}, J=13.8$ and $4.9 \mathrm{~Hz}, 1 \mathrm{H})$

${ }^{13} \mathrm{C}$ NMR $\left(100 \mathrm{MHz}, \mathrm{CDCl}_{3}\right) \delta 138.8,136.4,121.3,116.5,108.4,68.3,64.6,64.5,39.0,38.3,36.1$

IR (KBr) $v_{\max } 3428,2957,2890,1638,1413,1361,1249,1122,1047,1011,947,914 \mathrm{~cm}^{-1}$

MS (ESI, +ve) $m / z 219\left[(\mathrm{M}+\mathrm{Na})^{+}, 100 \%\right]$

HRMS (ESI, +ve) $m / z(\mathrm{M}+\mathrm{Na})^{+}$calcd for $\mathrm{C}_{11} \mathrm{H}_{16} \mathrm{NaO}_{3} 219.0997$, found 219.0995. 


\section{Compound 14}

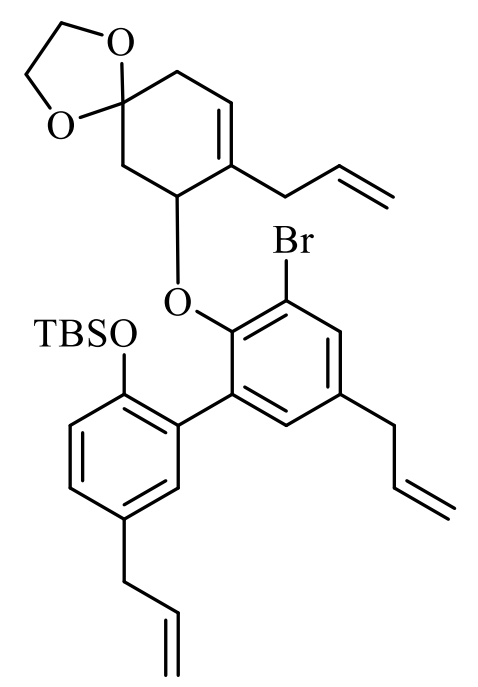

14

A magnetically stirred solution of compound $5(393 \mathrm{mg}, 2.00 \mathrm{mmol})$, compound 9 (1.10 g, $2.36 \mathrm{mmol})$ and $\mathrm{PPh}_{3}(621 \mathrm{mg}, 2.36 \mathrm{mmol})$ in dry THF $(45 \mathrm{~mL})$ maintained at $-78^{\circ} \mathrm{C}$ was treated, dropwise, with DEAD $(380 \mu \mathrm{l}, 2.36 \mathrm{mmol})$. The resulting orange mixture was maintained at $-78^{\circ} \mathrm{C}$ for $3 \mathrm{~h}$ then warmed to room temperature before being concentrated under reduced pressure. The residue thus obtained was subjected to flash chromatography (silica, 9:1 v/v hexane/ethyl acetate elution) and after concentration of the appropriate fractions $\left(R_{\mathrm{f}}=0.6\right.$ in $6: 1 \mathrm{v} / \mathrm{v}$ hexane/ethyl acetate) compound 14 (904 mg, 71\%) was obtained as a light-yellow oil.

${ }^{1}$ H NMR $\left(400 \mathrm{MHz}, \mathrm{CDCl}_{3}\right) \delta 7.34(\mathrm{~d}, J=2.4 \mathrm{~Hz}, 1 \mathrm{H}), 7.10(\mathrm{~d}, J=2.4 \mathrm{~Hz}, 1 \mathrm{H}), 7.05-6.97$ (complex m, 2H), $6.77(\mathrm{~d}, J=8.2 \mathrm{~Hz}, 1 \mathrm{H}), 5.96(\mathrm{~m}$, $2 \mathrm{H}), 5.79(\mathrm{~m}, 1 \mathrm{H}), 5.27(\mathrm{~m}, 1 \mathrm{H}), 5.14-4.92($ complex m, 6H), $4.48(\mathrm{~m}, 1 \mathrm{H}), 3.85(\mathrm{~m}, 1 \mathrm{H}), 3.75(\mathrm{~m}, 3 \mathrm{H}), 3.32(\mathrm{~m}, 4 \mathrm{H}), 3.00(\mathrm{dm}, J=16.7 \mathrm{~Hz}$, 1H), $2.55(\mathrm{dm}, J=16.7 \mathrm{~Hz}, 1 \mathrm{H}), 2.29(\mathrm{dm}, J=17.3 \mathrm{~Hz}, 1 \mathrm{H}), 2.05(\mathrm{dm}, J=17.3 \mathrm{~Hz}, 1 \mathrm{H}), 1.90-1.70$ (complex m, 2H), $0.78(\mathrm{~s}, 9 \mathrm{H}), 0.11(\mathrm{~m}, 6 \mathrm{H})$ 
${ }^{13}$ C NMR (100 MHz, $\left.\mathrm{CDCl}_{3}\right) \delta$ 151.2, 150.4, 137.7, 137.6, 136.9, 136.7, 135.7, 134.1, 132.3(1), 132.2(9), 132.1, 131.6, 129.8, 129.1, 120.5, $118.9,118.5,116.4,116.0,115.8,108.6,79.0,64.4,64.2,39.5,39.3,37.4,36.1,36.0,25.5,18.0,-4.2,-4.4$

IR $(\mathrm{KBr}) v_{\max } 3079,2955,2930,2893,2861,1639,1497,1251,1125,1055,1017,913,839,780 \mathrm{~cm}^{-1}$

MS (ESI, +ve) $m / z 661$ and $659\left[(\mathrm{M}+\mathrm{Na})^{+}, 100\right.$ and $\left.95 \%\right]$

HRMS (ESI, +ve) $m / z(\mathrm{M}+\mathrm{Na})^{+}$calcd for $\mathrm{C}_{35} \mathrm{H}_{45}{ }^{79} \mathrm{BrNaO}_{4} \mathrm{Si} 659.2168$, found 659.2169 .

\section{Compound 15}

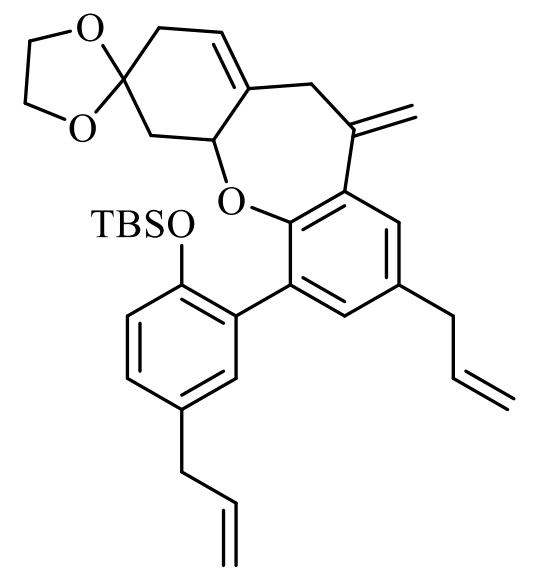

15

A magnetically stirred and degassed solution of compound $14(20 \mathrm{mg}, 0.032 \mathrm{mmol})$, Xphos $(6.2 \mathrm{mg}, 0.013 \mathrm{mmol}), \mathrm{Pd}(\mathrm{OAc}) 2(1.7 \mathrm{mg}, 0.007$ $\mathrm{mmol})$ and $\mathrm{Cs}_{2} \mathrm{CO}_{3}(31 \mathrm{mg}, 0.096 \mathrm{mmol})$ in toluene $(2 \mathrm{~mL})$ was heated at reflux for $48 \mathrm{~h}$. The ensuing black mixture was cooled then subjected to flash chromatography (silica, 9:1 v/v hexane/ethyl acetate elution) to afford, after concentration of the appropriate fractions $\left(R_{\mathrm{f}}=0.6\right.$ in $6: 1$ $\mathrm{v} / \mathrm{v}$ hexane/ethyl acetate), compound 15 (3 mg, 18\%) as a light-yellow oil. 
${ }^{1}$ H NMR $\left(400 \mathrm{MHz}, \mathrm{CDCl}_{3}\right) \delta 7.18(\mathrm{~d}, J=2.4 \mathrm{~Hz}, 1 \mathrm{H}), 7.08(\mathrm{~m}, 1 \mathrm{H}), 7.00(\mathrm{~m}, 2 \mathrm{H}), 6.78(\mathrm{~d}, J=8.1 \mathrm{~Hz}, 1 \mathrm{H}), 5.96(\mathrm{~m}, 2 \mathrm{H}), 5.40(\mathrm{broad} \mathrm{s}, 1 \mathrm{H})$, $5.30(\mathrm{~s}, 1 \mathrm{H}), 5.13-5.02$ (complex m, 5H), $4.75(\mathrm{~m}, 1 \mathrm{H}), 3.98-3.80$ (complex m, 4H), 3.45 (d, J = 14.0 Hz, $1 \mathrm{H}), 3.34(\mathrm{~m}, 4 \mathrm{H}), 3.14(\mathrm{~d}, J=14.0$ $\mathrm{Hz}, 1 \mathrm{H}), 2.35-2.06$ (complex m, 3H), $1.76(\mathrm{dd}, J=12.3$ and $10.4 \mathrm{~Hz}, 1 \mathrm{H}), 0.74(\mathrm{~s}, 9 \mathrm{H}), 0.00(\mathrm{~s}, 3 \mathrm{H}),-0.17(\mathrm{~s}, 3 \mathrm{H})$

${ }^{13}$ C NMR $\left(100 \mathrm{MHz}, \mathrm{CDCl}_{3}\right) \delta 153.5,151.4,144.8,139.6,138.1,137.8,133.2,131.6,131.5,131.4,131.2(1), 131.1(9), 130.8,128.2,127.7$, $119.7,117.8,115.7,115.6,113.4,108.7,79.5,64.7,64.5,42.4,39.7(2), 39.7(1), 37.8,35.9,25.6,18.1,-4.3,-4.7$

IR $(\mathrm{KBr}) v_{\max } 2957,2930,2893,2856,1639,1494,1240,1440,1240,1126,1034,909,839,780 \mathrm{~cm}^{-1}$

MS (ESI, +ve) $m / z 579\left[(\mathrm{M}+\mathrm{Na})^{+}, 95 \%\right], 574(100), 557(60)$.

HRMS $(\mathrm{ESI},+\mathrm{ve})(\mathrm{M}+\mathrm{Na})^{+}$calcd for $\mathrm{C}_{35} \mathrm{H}_{44} \mathrm{NaO}_{4} \mathrm{Si} 579.2907$, found 579.2914.

\section{Compound 17}

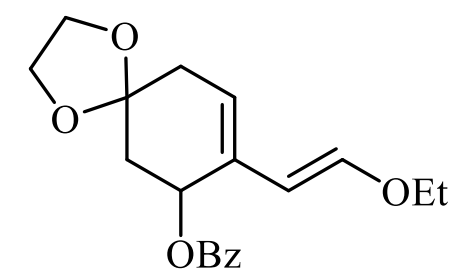

17

A magnetically stirred and degassed solution of compound $12(5.20 \mathrm{~g}, 12.7 \mathrm{mmol})$ and triethylamine $(13 \mathrm{~mL})$ in THF/water $(50 \mathrm{~mL}$ of a $9: 1$ mixture) was treated, sequentially, with $(E)-($ pin $) \mathrm{BCH}=\mathrm{CHOEt}^{4}(3.27 \mathrm{~g}, 16.5 \mathrm{mmol})$ and $\mathrm{PdCl}_{2} \mathrm{dppf} \cdot \mathrm{CH}_{2} \mathrm{Cl}_{2}(727 \mathrm{mg}, 7 \mathrm{~mol} \%)$. The resulting dark-red mixture was maintained at $22{ }^{\circ} \mathrm{C}$ for $1 \mathrm{~h}$ then cooled to $0{ }^{\circ} \mathrm{C}$ and treated with $\mathrm{H}_{2} \mathrm{O}_{2}\left(20 \mathrm{~mL}\right.$ of a $30 \%$ aqueous solution) and $\mathrm{NH}_{4} \mathrm{Cl}(20$ $\mathrm{mL}$ of a saturated aqueous solution). After $0.5 \mathrm{~h}$ at $0{ }^{\circ} \mathrm{C}$ the reaction mixture was warmed to $22{ }^{\circ} \mathrm{C}$ then extracted with diethyl ether $(3 \mathrm{x} 20 \mathrm{~mL})$. The combined organic phases were washed with brine $(1 \times 50 \mathrm{~mL})$ before being dried $\left(\mathrm{Na}_{2} \mathrm{SO}_{4}\right)$, filtered, and concentrated under reduced pressure. The residue thus obtained was subjected to flash chromatography (silica, 9:1 v/v hexane/ethyl acetate elution) to afford, after concentration of the appropriate fractions $\left(R_{f}=0.5 \mathrm{in} 2: 1 \mathrm{v} / \mathrm{v}\right.$ hexane/ethyl acetate), compound $17(3.57 \mathrm{~g}, 85 \%)$ as a light-yellow oil.

${ }^{1}$ H NMR $\left(400 \mathrm{MHz}, \mathrm{CDCl}_{3}\right) \delta 8.07(\mathrm{~m}, 2 \mathrm{H}), 7.55(\mathrm{~m}, 1 \mathrm{H}), 7.42(\mathrm{~m}, 2 \mathrm{H}), 6.47(\mathrm{~d}, J=12.9 \mathrm{~Hz}, 1 \mathrm{H}), 5.90(\mathrm{~m}, 1 \mathrm{H}), 5.72(\mathrm{~m}, 1 \mathrm{H}), 5.48(\mathrm{~d}, J=12.9$ $\mathrm{Hz}, 1 \mathrm{H}), 4.05-3.80($ complex $\mathrm{m}, 4 \mathrm{H}), 3.68(\mathrm{~m}, 2 \mathrm{H}), 2.52(\mathrm{dd}, J=18.4$ and $4.3 \mathrm{~Hz}, 1 \mathrm{H}), 2.41(\mathrm{dd}, J=18.4 \mathrm{and} 4.3 \mathrm{~Hz}, 1 \mathrm{H}), 2.28(\mathrm{~m}, 1 \mathrm{H}), 2.09$ $(\mathrm{m}, 1 \mathrm{H}), 1.17(\mathrm{t}, J=7.0 \mathrm{~Hz}, 3 \mathrm{H})$ 
${ }^{13} \mathrm{C}$ NMR $\left(100 \mathrm{MHz}, \mathrm{CDCl}_{3}\right) \delta 166.4,147.2,133.1,131.7,130.5,129.9,128.4,123.6,107.1,105.7,69.2,65.5,64.7,64.4,36.9,36.2,14.8$

IR $(\mathrm{KBr}) v_{\max } 2976,2880,1707,1657,1265,1107,946,713 \mathrm{~cm}^{-1}$

MS (ESI, +ve) $m / z 401(100 \%), 353\left[(\mathrm{M}+\mathrm{Na})^{+}, 60 \%\right]$

HRMS (ESI, +ve) $(\mathrm{M}+\mathrm{Na})^{+}$calcd for $\mathrm{C}_{19} \mathrm{H}_{22} \mathrm{NaO}_{5} 353.1365$, found 353.1362 .

\section{Compound 18}

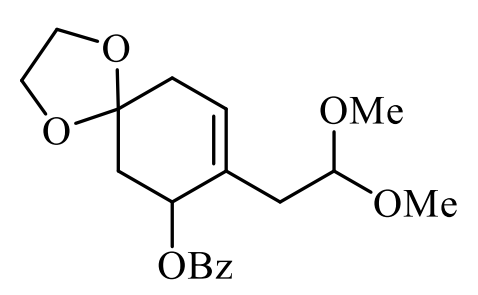

18

A magnetically stirred solution of compound $17(500 \mathrm{mg}, 1.53 \mathrm{mmol})$ in dry THF/methanol (11 mL of a 10:1 v/v mixture) was treated with $p$ $\mathrm{TsOH} \cdot \mathrm{H}_{2} \mathrm{O}(30 \mathrm{mg}, 0.15 \mathrm{mmol})$. The resulting mixture was maintained at $22{ }^{\circ} \mathrm{C}$ for $48 \mathrm{~h}$ then treated with $\mathrm{NaHCO}_{3}(10 \mathrm{~mL}$ of a saturated aqueous solution) and extracted with diethyl ether $(3 \times 10 \mathrm{~mL})$. The combined organic phases were washed with brine $(1 \times 20 \mathrm{~mL})$ before being dried $\left(\mathrm{Na}_{2} \mathrm{SO}_{4}\right)$, filtered, and concentrated under reduced pressure. The residue thus obtained was subjected to flash chromatography (silica, 9:1 $\mathrm{v} / \mathrm{v}$ hexane/ethyl acetate elution) to afford, after concentration of the appropriate fractions $\left(R_{f}=0.4\right.$ in $2: 1 \mathrm{v} / \mathrm{v}$ hexane/ethyl acetate), compound $18(352 \mathrm{mg}, 66 \%)$ as a light-yellow oil.

${ }^{1}$ H NMR $\left(400 \mathrm{MHz}, \mathrm{CDCl}_{3}\right) \delta 8.06(\mathrm{~d}, J=7.6 \mathrm{~Hz}, 2 \mathrm{H}), 7.56(\mathrm{~m}, 1 \mathrm{H}), 7.44(\mathrm{t}, J=7.6 \mathrm{~Hz}, 2 \mathrm{H}), 5.78(\mathrm{~m}, 2 \mathrm{H}), 4.50(\mathrm{t}, J=5.6 \mathrm{~Hz}, 1 \mathrm{H}), 3.97(\mathrm{~m}$, $4 \mathrm{H}), 3.28(\mathrm{~s}, 6 \mathrm{H}), 2.48(\mathrm{~m}, 2 \mathrm{H}), 2.41-2.28$ (complex m, 3H), 1.99 (dd, $J=13.2$ and $7.0 \mathrm{~Hz}, 1 \mathrm{H})$

${ }^{13}$ C NMR $\left(100 \mathrm{MHz}, \mathrm{CDCl}_{3}\right) \delta 166.3,133.1,131.9,130.6,129.8,128.5,126.3,107.4,103.7,71.7,64.7,64.6,53.5,53.1,37.2,36.4,36.1$

IR (KBr) $v_{\max } 2940,2889,2833,1712,1269,1111,1065,1047,713 \mathrm{~cm}^{-1}$

MS (ESI, +ve) $m / z 371\left[(\mathrm{M}+\mathrm{Na})^{+}, 100 \%\right]$ 
HRMS (ESI, +ve) $(\mathrm{M}+\mathrm{Na})^{+}$calcd for $\mathrm{C}_{19} \mathrm{H}_{24} \mathrm{NaO}_{6} 371.1471$, found 371.1479.

\section{Compound 19}

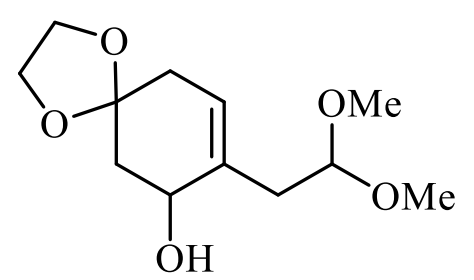

19

A magnetically stirred solution of compound $18(1.40 \mathrm{~g}, 4.02 \mathrm{mmol})$ in methanol $(50 \mathrm{~mL})$ was treated with $\mathrm{NaOH}(20 \mathrm{~mL}$ of a $3 \mathrm{M}$ aqueous solution, $60 \mathrm{mmol})$ and then heated at reflux for $0.5 \mathrm{~h}$. The resulting solution was cooled then treated with water $(300 \mathrm{~mL})$ and extracted with ethyl acetate $(5 \times 60 \mathrm{~mL})$. The combined organic phases were washed with brine $(1 \times 50 \mathrm{~mL})$ before dried $\left(\mathrm{Na}_{2} \mathrm{SO}_{4}\right)$, filtered, and concentrated under reduced pressure to give compound $19(943 \mathrm{mg}, 96 \%)$ as a light-yellow oil. This material was used without further purification in the next step of the reaction sequence.

$\boldsymbol{R}_{\mathbf{f}}=0.3$ (in $1: 2 \mathrm{v} / \mathrm{v}$ hexane/ethyl acetate)

${ }^{1} \mathbf{H}$ NMR $\left(400 \mathrm{MHz}, \mathrm{CDCl}_{3}\right) \delta 5.52(\mathrm{t}, J=3.9 \mathrm{~Hz}, 1 \mathrm{H}), 4.55(\mathrm{~m}, 1 \mathrm{H}), 4.20(\mathrm{~m}, 1 \mathrm{H}), 4.05-3.90($ complex m, 4H), 3.36 (s, 3H), $3.34(\mathrm{~s}, 3 \mathrm{H}), 3.24$ $(\mathrm{d}, J=9.5 \mathrm{~Hz}, 1 \mathrm{H}), 2.60(\mathrm{~m}, 1 \mathrm{H}), 2.39(\mathrm{dd}, J=14.6$ and $4.7 \mathrm{~Hz}, 1 \mathrm{H}), 2.31(\mathrm{~m}, 2 \mathrm{H}), 2.05(\mathrm{dd}, J=13.5 \mathrm{and} 4.7 \mathrm{~Hz}, 1 \mathrm{H}), 1.99(\mathrm{dd}, J=13.5 \mathrm{and}$ 4.7 Hz, 1H)

${ }^{13}$ C NMR (100 MHz, $\left.\mathrm{CDCl}_{3}\right) \delta 135.9,123.4,108.2,104.1,68.6,64.5(3), 64.5(2), 53.6,52.8,39.2,37.3,36.2$

IR (KBr) $v_{\max } 3452,2954,2931,2893,2832,1362,1119,1042 \mathrm{~cm}^{-1}$

MS (ESI, +ve) $m / z 267\left[(\mathrm{M}+\mathrm{Na})^{+}, 100 \%\right]$

HRMS (ESI, +ve) $(\mathrm{M}+\mathrm{Na})^{+}$calcd for $\mathrm{C}_{12} \mathrm{H}_{20} \mathrm{NaO}_{5} 267.1208$, found 267.1205 


\section{Compound 20}

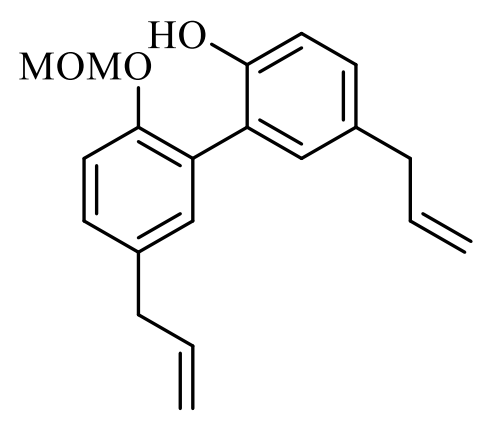

20

A magnetically stirred solution of magnolol $(1.00 \mathrm{~g}, 3.76 \mathrm{mmol})$ and triethylamine $(0.74 \mathrm{~mL}, 5.30 \mathrm{mmol})$ in dry dichloromethane $(5 \mathrm{~mL})$ was treated with a freshly prepared solution of $\mathrm{MOMCl}^{5}(10 \mathrm{~mL}$ of a $1 \mathrm{M}$ solution in dichloromethane, 10.0 mmol). The resulting light-yellow solution was maintained at $22{ }^{\circ} \mathrm{C}$ for $2 \mathrm{~h}$ then treated with $\mathrm{NH}_{4} \mathrm{Cl}(10 \mathrm{~mL}$ of a saturated aqueous solution). After a further $0.25 \mathrm{~h}$ the reaction mixture was extracted with diethyl ether $(3 \times 10 \mathrm{~mL})$ and the combined organic phases washed with brine $(1 \mathrm{x} 20 \mathrm{~mL})$ before being dried $\left(\mathrm{Na}_{2} \mathrm{SO}_{4}\right)$, filtered, and concentrated under reduced pressure to give compound $\mathbf{2 0}(1.10 \mathrm{~g}, 94 \%)$ as a light-yellow oil. This material was used without further purification in the next step of the reaction sequence.

$\boldsymbol{R}_{\mathbf{f}}=0.8($ in $7: 3 \mathrm{v} / \mathrm{v}$ hexane/ethyl acetate)

${ }^{1}$ H NMR (400 MHz, $\left.\mathrm{CDCl}_{3}\right) \delta$ 7.22-7.06 (complex m, 5H), $6.96(\mathrm{~d}, J=8.2 \mathrm{~Hz}, 1 \mathrm{H}), 6.05(\mathrm{~s}, 1 \mathrm{H}), 5.98(\mathrm{~m}, 2 \mathrm{H}), 5.12(\mathrm{~s}, 2 \mathrm{H}), 5.10-5.04$ (complex m, 4H), 3.41-3.39 (complex m, 4H), 3.37 (s, 3H)

${ }^{13}$ C NMR $\left(100 \mathrm{MHz}, \mathrm{CDCl}_{3}\right) \delta 152.0(4), 152.0(2), 138.0,137.4,135.5,132.6,132.5,131.3,129.5(3), 129.5(0), 128.5,126.2,117.3,116.8$, $116.1,115.7,96.2,56.6,39.6(4), 39.6(0)$

IR $(\mathrm{KBr}) v_{\max } 3405,2979,2902,1636,1498,1229,1150,986,907,820 \mathrm{~cm}^{-1}$

MS (ESI, +ve) $m / z 333\left[(\mathrm{M}+\mathrm{Na})^{+}, 100 \%\right]$

HRMS (ESI, +ve) $(\mathrm{M}+\mathrm{Na})^{+}$calcd for $\mathrm{C}_{20} \mathrm{H}_{22} \mathrm{NaO}_{3} 333.1467$, found 333.1463. 


\section{Compound 21}

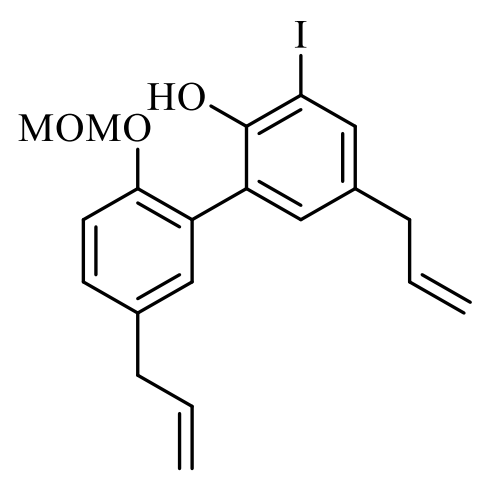

21

A magnetically stirred solution of compound $20(1.10 \mathrm{~g}, 3.50 \mathrm{mmol})$ in dichloromethane $(100 \mathrm{~mL})$ was treated with $\left(\mathrm{collidene}_{2} \mathrm{IPF}_{6}{ }^{6}(2.00 \mathrm{~g}\right.$, $4.00 \mathrm{mmol}$ ). The ensuing green mixture was maintained at $22^{\circ} \mathrm{C}$ for $0.1 \mathrm{~h}$ then concentrated under reduced pressure. The residue thus obtained was subjected to flash chromatography (silica, 9:1 v/v hexane/ethyl acetate elution) to afford, after concentration of the appropriate fractions $\left(R_{f}\right.$ $=0.8$ in $2: 1 \mathrm{v} / \mathrm{v}$ hexane/ethyl acetate), compound $21(1.50 \mathrm{~g}, 98 \%)$ as a light-yellow oil.

${ }^{1} \mathbf{H}$ NMR $\left(400 \mathrm{MHz}, \mathrm{CDCl}_{3}\right) \delta 7.58(\mathrm{~d}, J=2.1 \mathrm{~Hz}, 1 \mathrm{H}), 7.18(\mathrm{~m}, 2 \mathrm{H}), 7.09(\mathrm{~d}, J=1.8 \mathrm{~Hz}, 1 \mathrm{H}), 7.04(\mathrm{~d}, J=2.1 \mathrm{~Hz}, 1 \mathrm{H}), 6.33(\mathrm{~s}, 1 \mathrm{H}), 5.95(\mathrm{~m}$, $2 \mathrm{H}), 5.15(\mathrm{~s}, 2 \mathrm{H}), 5.12-5.05$ (complex m, 4H), $3.38(\mathrm{~d}, J=6.7 \mathrm{~Hz}, 2 \mathrm{H}), 3.36(\mathrm{~s}, 3 \mathrm{H}), 3.33$ (d, $J=6.7 \mathrm{~Hz}, 2 \mathrm{H})$

${ }^{13} \mathbf{C}$ NMR $\left(100 \mathrm{MHz}_{\mathrm{CDCl}}\right) \delta 152.2,150.9,138.6,137.3,137.2,135.1,134.2,132.2,132.0,129.9,128.0,126.2,116.5,116.3,116.2,96.0$, $86.1,56.6,39.5,39.0$

IR (KBr) $v_{\max } 3365,3077,2919,2853,1639,1496,1458,1229,1155,1078,997,912 \mathrm{~cm}^{-1}$

MS (ESI, +ve) $m / z 459\left[(\mathrm{M}+\mathrm{Na}]^{+}, 100 \%\right]$

HRMS (ESI, +ve) $m / z(\mathrm{M}+\mathrm{Na})^{+}$calcd for $\mathrm{C}_{20} \mathrm{H}_{21} \mathrm{INaO}_{3} 459.0433$, found 459.0432 . 


\section{Compound 22}

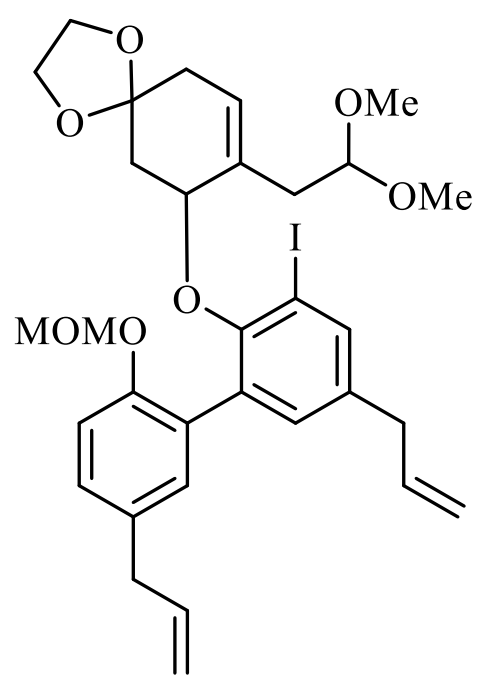

22

A magnetically stirred solution of compound 19 (940 mg, $3.85 \mathrm{mmol})$, compound 21 (2.00 g, $4.62 \mathrm{mmol})$ and $\mathrm{PPh}_{3}(1.20 \mathrm{~g}, 4.62 \mathrm{mmol})$ in dry THF $(90 \mathrm{~mL})$ maintained at $0{ }^{\circ} \mathrm{C}$ was treated, dropwise, with DEAD $(740 \mu \mathrm{L}, 4.62 \mathrm{mmol})$. The resulting orange mixture was maintained at $0{ }^{\circ} \mathrm{C}$ for $0.5 \mathrm{~h}$ then warmed to room temperature before being concentrated under reduced pressure. The residue thus obtained was subjected to flash chromatography (silica, 9:1 v/v hexane/ethyl acetate elution) to afford, after concentration of the appropriate fractions $\left(R_{f}=0.5\right.$ in $2: 1 \mathrm{v} / \mathrm{v}$ hexane/ethyl acetate), compound $22(1.99 \mathrm{~g}, 78 \%)$ as a clear, light-yellow oil.

${ }^{1}$ H NMR $\left(400 \mathrm{MHz}, \mathrm{CDCl}_{3}\right) \delta 7.59(\mathrm{~d}, J=2.2 \mathrm{~Hz}, 1 \mathrm{H}), 7.11(\mathrm{~m}, 2 \mathrm{H}), 7.08(\mathrm{~m}, 1 \mathrm{H}), 7.03(\mathrm{~d}, J=2.2 \mathrm{~Hz}, 1 \mathrm{H}), 5.94(\mathrm{~m}, 2 \mathrm{H}), 5.43(\mathrm{~d}, J=5.1 \mathrm{~Hz}$, $1 \mathrm{H}), 5.13-5.03$ (complex m, 6H), $4.61(\mathrm{t}, J=5.6 \mathrm{~Hz}, 1 \mathrm{H}), 4.42(\mathrm{~m}, 1 \mathrm{H}), 3.90-3.75$ (complex m, 4H), 3.41-3.29 (complex m, 13H), 2.36-2.32 (complex m, 3H), $2.06(\mathrm{~m}, 1 \mathrm{H}), 1.80(\mathrm{~m}, 2 \mathrm{H})$

${ }^{13}$ C NMR (100 MHz, $\left.\mathrm{CDCl}_{3}\right) \delta 152.8,152.3,138.5,137.5,136.9,136.6,133.5,132.9,132.1,131.4,129.5,128.3,123.2,116.5,116.0,115.4$, 108.5(3), 108.5(2), 103.9, 95.1, 94.6, 78.7, 64.3(4), 64.3(0), 56.1, 53.9, 53.1, 39.5, 39.0, 37.2, 36.3, 36.0 (one signal obscured or overlapping) 
IR $(\mathrm{KBr}) v_{\max } 2969,2921,2898,2830,1642,1497,1438,1225,113,1042,999 \mathrm{~cm}^{-1}$

MS (ESI, +ve) $m / z 685\left[(\mathrm{M}+\mathrm{Na})^{+}, 100 \%\right]$

HRMS (ESI, +ve) $m / z(\mathrm{M}+\mathrm{Na})^{+}$calcd for $\mathrm{C}_{32} \mathrm{H}_{39} \mathrm{INaO}_{7} 685.1638$, found 685.1636.

\section{Compound 23}

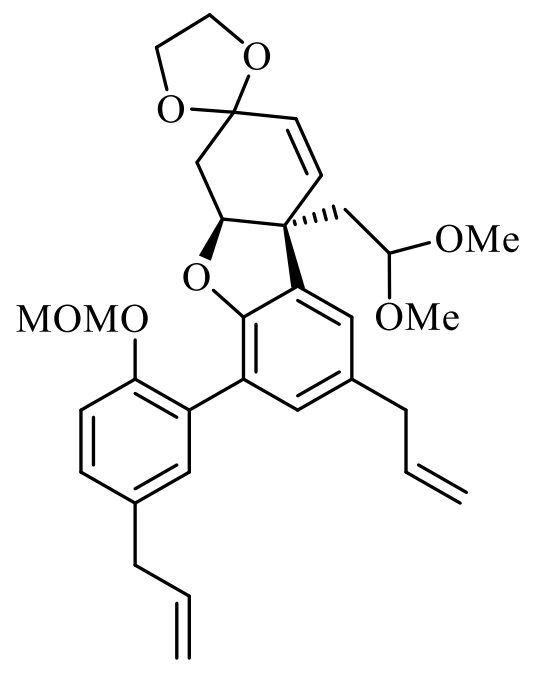

23

A magnetically stirred and thoroughly degassed solution of compound $\mathbf{2 2}(2.00 \mathrm{~g}, 3.02 \mathrm{mmol})$ in dry toluene $(111 \mathrm{~mL}) \mathrm{maintained}$ under a nitrogen atmosphere was treated, sequentially, with $\mathrm{Pd}(\mathrm{OAc})_{2}(69 \mathrm{mg}, 10 \mathrm{~mol} \%)$, dppp (255 mg, $\left.20 \mathrm{~mol} \%\right)$ and $\mathrm{Ag}_{2} \mathrm{CO}_{3}(2.60 \mathrm{~g}, 9.30 \mathrm{mmol})$. The resulting heterogeneous mixture was heated at reflux for $1 \mathrm{~h}$ then cooled, filtered through a pad of diatomaceous earth and the filtrate evaporated under reduced pressure. The residue thus obtained was subjected to flash chromatography (silica, 9:1 v/v hexane/ethyl acetate elution) to afford, after concentration of the appropriate fractions $\left(R_{f}=0.7\right.$ in $1: 1 \mathrm{v} / \mathrm{v}$ hexane/ethyl acetate), compound $23(1.49 \mathrm{~g}, 92 \%)$ as a light-yellow oil. 
${ }^{1}$ H NMR $\left(400 \mathrm{MHz}, \mathrm{CDCl}_{3}\right) \delta 7.20(\mathrm{~m}, 1 \mathrm{H}), 7.15(\mathrm{~m}, 1 \mathrm{H}), 7.02(\mathrm{~m}, 1 \mathrm{H}), 7.09(\mathrm{~m}, 1 \mathrm{H}), 6.88(\mathrm{~d}, J=1.9 \mathrm{~Hz}, 1 \mathrm{H}), 6.03-5.91(\mathrm{complex} \mathrm{m}, 3 \mathrm{H})$, $5.76(\mathrm{~d}, J=10.1 \mathrm{~Hz}, 1 \mathrm{H}), 5.11-4.99($ complex m, 7H), $4.44(\mathrm{~m}, 1 \mathrm{H}), 3.97(\mathrm{~m}, 4 \mathrm{H}), 3.39(\mathrm{~s}, 3 \mathrm{H}), 3.37-3.34(\mathrm{complex} \mathrm{m}, 4 \mathrm{H}), 3.28(\mathrm{~s}, 3 \mathrm{H}), 3.27$ (s, 3H), 2.22-1.96 (complex m, 4H)

${ }^{13}$ C NMR (100 MHz, $\left.\mathrm{CDCl}_{3}\right) \delta 154.2,153.2,138.0,137.8,133.5(3), 133.5(0), 132.5,132.1,131.8,130.6,128.8,127.5(1), 127.5(0), 122.3$, 121.6, 116.0, 115.7, 115.6, 104.4, 102.1, 95.6, 84.3, 64.8, 64.6, 56.0, 52.9, 52.6, 47.3, 42.9, 39.9, 39.6, 35.8

IR $(\mathrm{KBr}) v_{\max } 2977,2957,2896,2832,1720,1642,1502,1461,1121,1012,920 \mathrm{~cm}^{-1}$

MS (ESI, +ve) $m / z 557\left[(\mathrm{M}+\mathrm{Na})^{+}, 100 \%\right]$

HRMS (ESI, +ve) $(\mathrm{M}+\mathrm{H})^{+}$calcd for $\mathrm{C}_{32} \mathrm{H}_{39} \mathrm{O}_{7}$ 535.2696, found 535.2698.

\section{Compound 24}

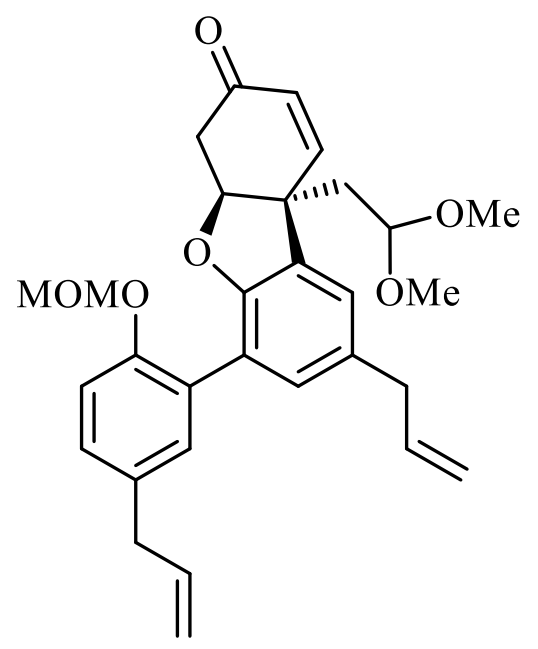

24

A magnetically stirred solution of compound $23(1.48 \mathrm{~g}, 2.77 \mathrm{mmol})$ in THF $(80 \mathrm{~mL})$ was treated with $\mathrm{HCl}(20 \mathrm{~mL}$ of a $1 \mathrm{M}$ aqueous solution, $20 \mathrm{mmol})$. The resulting mixture was maintained at $22{ }^{\circ} \mathrm{C}$ for $2 \mathrm{~h}$ then treated with $\mathrm{NaHCO}_{3}(30 \mathrm{~mL}$ of a saturated aqueous solution) and 
extracted with diethyl ether $(3 \times 30 \mathrm{~mL})$. The combined organic phases were washed with brine $(1 \times 50 \mathrm{~mL})$ then dried $\left(\mathrm{Na}_{2} \mathrm{SO}_{4}\right)$, filtered, and concentrated under reduced pressure to give compound $24(1.35 \mathrm{~g}, 99 \%)$ as a light-yellow oil. This material was used without purification in the next step of the reaction sequence.

$\boldsymbol{R}_{\mathbf{f}}=0.6($ in $1: 1 \mathrm{v} / \mathrm{v}$ hexane/ethyl acetate)

${ }^{1}$ H NMR $\left(400 \mathrm{MHz}, \mathrm{CDCl}_{3}\right) \delta 7.22-7.05$ (complex m, 4H), $6.99(\mathrm{~d}, J=1.9 \mathrm{~Hz}, 1 \mathrm{H}), 6.53(\mathrm{dd}, J=10.2 \mathrm{and} 1.8 \mathrm{~Hz}, 1 \mathrm{H}), 6.03(\mathrm{~d}, J=10.2 \mathrm{~Hz}$, $1 \mathrm{H}), 5.98(\mathrm{~m}, 2 \mathrm{H}), 5.16-4.97$ (complex m, 6H), $4.94(\mathrm{~m}, 1 \mathrm{H}), 4.55$ (m, 1H), 3.44-3.30 (complex m, 13H), 2.94 (broad s, $2 \mathrm{H}), 2.40(\mathrm{dd}, J=14.6$ and $4.1 \mathrm{~Hz}, 1 \mathrm{H}), 2.28(\mathrm{dd}, J=14.6$ and $6.8 \mathrm{~Hz}, 1 \mathrm{H})$

${ }^{13}$ C NMR $\left(100 \mathrm{MHz}, \mathrm{CDCl}_{3}\right) \delta 196.0,154.8,153.6,148.4,137.6(4), 137.6(1), 137.6(0), 133.5,133.2,131.4,131.2(3), 131.2(2), 129.2,127.1$, $126.9,121.9,116.0,115.8,115.6,101.8,95.5,85.5,56.0,53.1,52.8,47.0,39.9,39.8,39.5,38.6$

IR (KBr) $v_{\max } 2901,2830,1682,1499,1468,1122,1073,905,724 \mathrm{~cm}^{-1}$

MS (ESI, +ve) $m / z 513\left[(\mathrm{M}+\mathrm{Na})^{+}, 100 \%\right]$

HRMS (ESI, +ve) $(\mathrm{M}+\mathrm{Na})^{+}$calcd for $\mathrm{C}_{30} \mathrm{H}_{34} \mathrm{NaO}_{6}$ 513.2253, found 513.2259. 


\section{Compound 25}

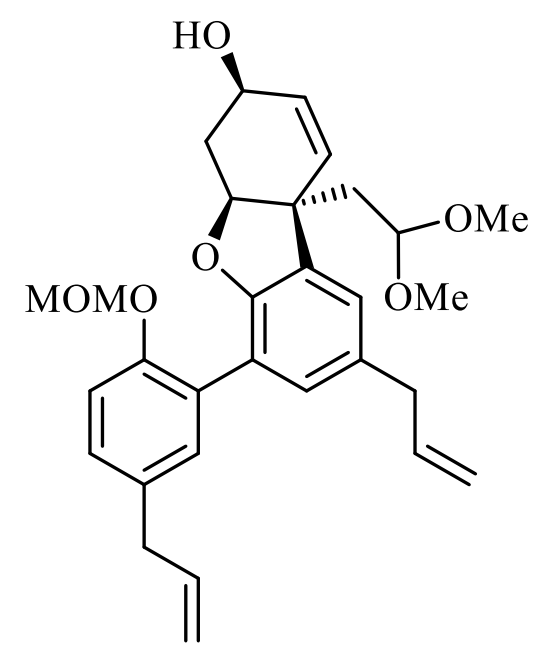

25

A magnetically stirred solution of compound $24(1.35 \mathrm{~g}, 2.75 \mathrm{mmol})$ in methanol $(100 \mathrm{~mL})$ was treated, in one portion, with polymer-bound borohydride (7.0 g of Amberlyst A26, $2.5 \mathrm{mmol}$ active hydride per g, $17.5 \mathrm{mmol}$ ). The resulting mixture was stirred at $22{ }^{\circ} \mathrm{C}$ for $24 \mathrm{~h}$ then filtered through a sintered glass funnel and the retained solids washed with methanol $(6 \mathrm{x} 40 \mathrm{~mL})$. The combined filtrates were concentrated under reduced pressure to give compound $25(1.29 \mathrm{~g}, 95 \%)$ as a pale yellow oil and as a 10:1 mixture of diastereoisomers. This material was used without purification in the next step of the reaction sequence.

$\boldsymbol{R}_{\mathbf{f}}=0.2$ (minor diasteroisomer) and 0.4 (in $2: 1 \mathrm{v} / \mathrm{v}$ hexane/ethyl acetate)

${ }^{1} \mathbf{H}$ NMR $\left(400 \mathrm{MHz}, \mathrm{CDCl}_{3}\right) \delta$ (major diastereoisomer) 7.14-7.08 (complex m, 3H), 6.96 (m, 2H), 6.09-5.91 (complex m, 3H), 5.54 (d, $J=10.0$ $\mathrm{Hz}, 1 \mathrm{H}), 5.12(\mathrm{~m}, 1 \mathrm{H}), 5.09-5.00($ complex m, 4H), $4.86(\mathrm{~m}, 1 \mathrm{H}), 4.81(\mathrm{~d}, J=6.7 \mathrm{~Hz}, 1 \mathrm{H}), 4.46(\mathrm{~m}, 1 \mathrm{H}), 4.13(\mathrm{~m}, 1 \mathrm{H}), 3.49(\mathrm{~d}, J=11.4 \mathrm{~Hz}$, $1 \mathrm{H}), 3.38(\mathrm{~m}, 4 \mathrm{H}), 3.29(\mathrm{~s}, 3 \mathrm{H}), 3.28(\mathrm{~s}, 3 \mathrm{H}), 3.09(\mathrm{~s}, 3 \mathrm{H}), 2.52(\mathrm{~m}, 1 \mathrm{H}), 2.26-2.01$ (complex m, 3H) 
${ }^{13} \mathbf{C}$ NMR $\left(100 \mathrm{MHz}, \mathrm{CDCl}_{3}\right) \delta$ (major diastereoisomer) 153.9, 153.2, 137.8, 137.4, 134.9, 133.7, 133.0, 131.2, 131.0, 130.0, 129.2(3), 129.2(1), $128.2,122.2,121.7,118.4,115.9,115.7,101.8,96.9,85.9,61.6,56.0,52.5(3), 52.4(8), 46.5,39.8,39.6,39.5,30.6$

IR (KBr) $v_{\max } 3493,2916,2827,1639,1499,1468,1119,1043,992,905,798 \mathrm{~cm}^{-1}$

MS (ESI, +ve) $m / z 515\left[(\mathrm{M}+\mathrm{Na})^{+}, 100 \%\right]$

HRMS (ESI, +ve) $m / z(\mathrm{M}+\mathrm{Na})^{+}$calcd for $\mathrm{C}_{30} \mathrm{H}_{36} \mathrm{NaO}_{6}$ 515.2410, found 515.2408.

\section{Compound 26}

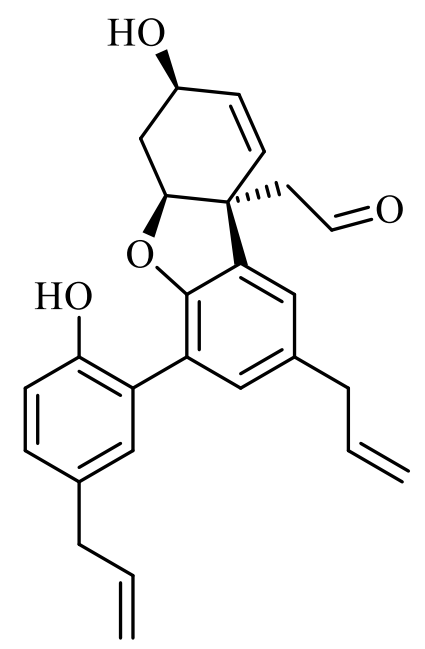

26

A magnetically stirred solution of compound 25 (1.28 g, $2.60 \mathrm{mmol})$ in THF (66 mL) was treated with $\mathrm{HCl}(33 \mathrm{~mL}$ of a $1 \mathrm{M}$ aqueous solution, $33 \mathrm{mmol})$ then heated at reflux for $8 \mathrm{~h}$. The resulting mixture was cooled then treated with $\mathrm{NaHCO}_{3}(40 \mathrm{~mL}$ of a saturated aqueous solution) and extracted with ethyl acetate $(3 \times 40 \mathrm{~mL})$. The combined organic phases were washed with brine $(1 \times 20 \mathrm{~mL})$ before being dried $\left(\mathrm{Na}_{2} \mathrm{SO}_{4}\right)$, filtered, and concentrated under reduced pressure. The residue thus obtained was subjected to flash chromatography (silica, 1:1 v/v hexane/ethyl 
acetate elution) and thus affording, after concentration of the appropriate fractions $\left(R_{f}=0.2\right.$ in $1: 1 \mathrm{v} / \mathrm{v}$ hexane/ethyl acetate), compound $\mathbf{2 6}$ ( 858 $\mathrm{mg}, 82 \%)$ as a pale-yellow solid, $\mathrm{mp}=70-72{ }^{\circ} \mathrm{C}$.

${ }^{1}$ H NMR $\left(400 \mathrm{MHz}, \mathrm{CDCl}_{3}\right) \delta 9.79(\mathrm{t}, J=2.6 \mathrm{~Hz}, 1 \mathrm{H}), 8.20($ broad s, $1 \mathrm{H}), 7.15-6.95($ complex m, 4H), $6.85(\mathrm{~d}, J=8.2 \mathrm{~Hz}, 1 \mathrm{H}), 5.99(\mathrm{~m}, 3 \mathrm{H})$, $5.74(\mathrm{dd}, J=10.0$ and $1.4 \mathrm{~Hz}, 1 \mathrm{H}), 5.17-5.06$ (complex m, 4H), 4.81 (broad s, $1 \mathrm{H}), 4.26($ broad s, $2 \mathrm{H}), 3.40(\mathrm{~d}, J=6.6 \mathrm{~Hz}, 2 \mathrm{H}), 3.37(\mathrm{~d}, J=6.6$ $\mathrm{Hz}, 2 \mathrm{H}), 2.94(\mathrm{dd}, J=16.0$ and $2.6 \mathrm{~Hz}, 1 \mathrm{H}), 2.83(\mathrm{dd}, J=16.0$ and $2.6 \mathrm{~Hz}, 1 \mathrm{H}), 2.63(\mathrm{dd}, J=15.2$ and $3.7 \mathrm{~Hz}, 1 \mathrm{H}), 2.06(\mathrm{~m}, 1 \mathrm{H})$

${ }^{13}$ C NMR $\left(100 \mathrm{MHz}, \mathrm{CDCl}_{3}\right) \delta 200.2,153.4,152.4,137.9,137.6,134.0,132.0,131.6,130.8,130.7,130.5,129.4,127.8,124.5,122.2(3)$, $122.1(9), 116.2,116.1,115.7,85.0,61.3,49.4,46.6,39.9,39.5,30.3$

IR $(\mathrm{KBr}) v_{\max } 3436,3187,3019,2903,2829,1721,1640,1467,1407,1213,1053,756 \mathrm{~cm}^{-1}$

MS (ESI, -ve) $m / z 401\left[(\mathrm{M}-\mathrm{H})^{-}, 100 \%\right]$

HRMS (ESI , -ve) $(\mathrm{M}-\mathrm{H})^{-}$calcd for $\mathrm{C}_{26} \mathrm{H}_{25} \mathrm{O}_{4} 401.1753$, found 401.1763 .

\section{Compound 27}

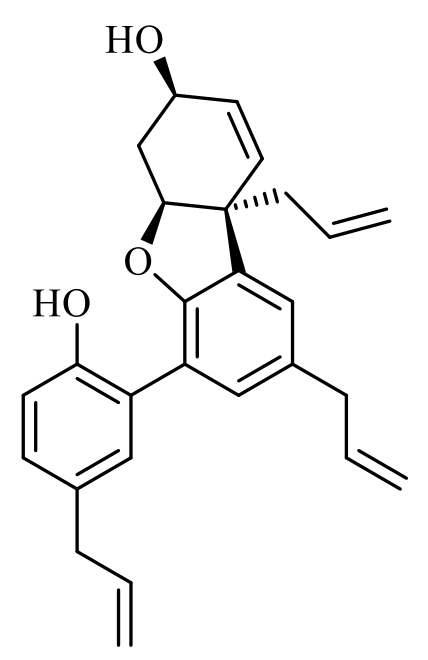

27 
A magnetically stirred solution of methyltriphenylphosphonium bromide $(3.93 \mathrm{~g}, 11.0 \mathrm{mmol})$ in dry THF (70 mL) was cooled to $0{ }^{\circ} \mathrm{C}$ then treated with $n$-BuLi $(6.25 \mathrm{~mL}$ of a $1.6 \mathrm{M}$ solution in hexanes, $10.0 \mathrm{mmol})$. The resulting orange-colored mixture was maintained at this temperature for $0.5 \mathrm{~h}$ then treated with a solution of aldehyde $26(700 \mathrm{mg}, 1.74 \mathrm{mmol})$ in dry THF (30 mL). The resulting yellow mixture was maintained at $0{ }^{\circ} \mathrm{C}$ for $0.5 \mathrm{~h}$ then treated with $\mathrm{HCl}(30 \mathrm{~mL}$ of a $1 \mathrm{M}$ aqueous solution) and extracted with diethyl ether (3 x $20 \mathrm{~mL})$. The combined organic phases were washed with brine $(1 \times 20 \mathrm{~mL})$ then dried $\left(\mathrm{Na}_{2} \mathrm{SO}_{4}\right)$, filtered, and concentrated under reduced pressure. The residue thus obtained was subjected to flash chromatography (silica, 9:1 v/v hexane/ethyl acetate elution) to afford, after concentration of the appropriate fractions $\left(\mathrm{R}_{f}=0.6 \mathrm{in} 1: 1 \mathrm{v} / \mathrm{v}\right.$ hexane/ethyl acetate), compound $27(502 \mathrm{mg}, 72 \%)$ as a white, crystalline solid, $\mathrm{mp}=110-112{ }^{\circ} \mathrm{C}$.

${ }^{1}$ H NMR $\left(400 \mathrm{MHz}, \mathrm{CDCl}_{3}\right) \delta$ 7.16-6.96 (complex m, 4H), 6.88 (d, $\left.J=8.2 \mathrm{~Hz}, 1 \mathrm{H}\right), 6.05-5.92$ (complex m, 3H), 5.79-5.62 (complex m, 2H), 5.18-5.01 (complex m, 6H), 4.77 (broad s, $1 \mathrm{H}), 4.21(\mathrm{~m}, 1 \mathrm{H}), 3.40(\mathrm{~d}, J=6.9 \mathrm{~Hz}, 2 \mathrm{H}), 3.36$ (d, $J=6.9 \mathrm{~Hz}, 2 \mathrm{H}), 2.63(\mathrm{dd}, J=14.2 \mathrm{and} 7.0 \mathrm{~Hz}$, $1 \mathrm{H}), 2.53(\mathrm{~m}, 2 \mathrm{H}), 1.97(\mathrm{~m}, 1 \mathrm{H})$ (signals due to the hydroxyl group protons not observed)

${ }^{13}$ C NMR $\left(100 \mathrm{MHz}, \mathrm{CDCl}_{3}\right) \delta 153.6,152.5,138.0,137.9,133.6,133.4,133.1,132.5,131.8,130.8,129.9,129.2,127.2,124.8,122.2,121.8$, $118.7,116.3,115.8,115.6,85.0,61.6,48.1,41.1,40.0,39.6,31.0$

IR $(\mathrm{KBr}) v_{\max } 3436,3181,3076,2918,1637,1507,1469,1416,1222,1043,987,910,732 \mathrm{~cm}^{-1}$

MS (ESI, +ve) $m / z 439(80 \%), 423\left[(\mathrm{M}+\mathrm{Na})^{+}, 100\right]$

HRMS (ESI, +ve) $m / z(\mathrm{M}+\mathrm{Na})^{+}$calcd for $\mathrm{C}_{27} \mathrm{H}_{28} \mathrm{NaO}_{3} 423.1936$, found 423.1934 . 


\section{Compound ( \pm )-1}

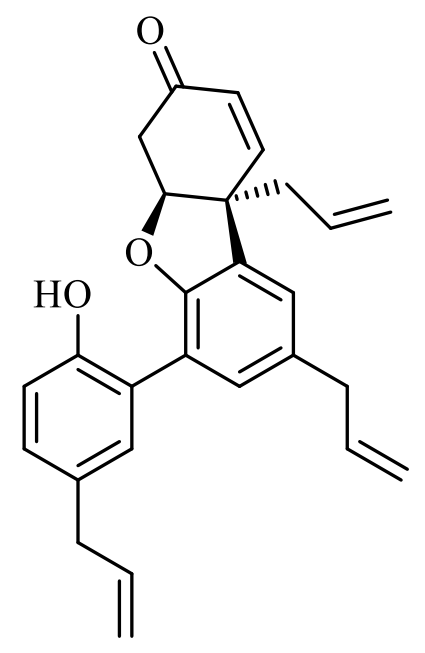

1

A magnetically stirred solution of compound $27(50 \mathrm{mg}, 0.125 \mathrm{mmol})$ in dry dichloromethane $(3.5 \mathrm{~mL})$ and dry DMSO $(1.5 \mathrm{~mL})$ was treated with triethylamine $(75 \mu 1,0.50 \mathrm{mmol})$ and cooled to $0^{\circ} \mathrm{C}$. The resulting mixture was treated with $\mathrm{SO}_{3} \cdot \mathrm{pyr}(60 \mathrm{mg}, 0.375 \mathrm{mmol})$, maintained at 0 ${ }^{\circ} \mathrm{C}$ for $3 \mathrm{~h}$ then treated with further portions of triethylamine $(75 \mu \mathrm{l}, 0.500 \mathrm{mmol})$ and $\mathrm{SO}_{3} \bullet$ pyr $(60 \mathrm{mg}, 0.375 \mathrm{mmol})$. After a further $0.5 \mathrm{~h}$ the reaction mixture was treated with $\mathrm{HCl}(5 \mathrm{~mL}$ of a $1 \mathrm{M}$ aqueous solution) and extracted with dichloromethane $(2 \times 5 \mathrm{~mL})$. The combined organic phases were washed with brine $(1 \times 5 \mathrm{~mL})$ then dried $\left(\mathrm{Na}_{2} \mathrm{SO}_{4}\right)$, filtered, and concentrated under reduced pressure. The residue thus obtained was subjected to flash chromatography (silica, $9: 1 \mathrm{v} / \mathrm{v}$ hexane/ethyl acetate elution) to afford, after concentration of the appropriate fractions $\left(\mathrm{R}_{f}=0.4\right.$ in $2: 1 \mathrm{v} / \mathrm{v}$ hexane/ethyl acetate), compound $( \pm)-\mathbf{1}(40 \mathrm{mg}, 80 \%)$ as a white, amorphous solid.

${ }^{1}$ H NMR $\left[400 \mathrm{MHz},\left(\mathrm{CD}_{3}\right)_{2} \mathrm{CO}\right] \delta 7.67(\mathrm{~s}, 1 \mathrm{H}), 7.23(\mathrm{~d}, J=1.8 \mathrm{~Hz}, 1 \mathrm{H}), 7.13(\mathrm{~d}, J=1.8 \mathrm{~Hz}, 1 \mathrm{H}), 7.08(\mathrm{~d}, J=2.3 \mathrm{~Hz}, 1 \mathrm{H}), 7.01(\mathrm{dd}, J=8.2 \mathrm{and}$ $2.3 \mathrm{~Hz}, 1 \mathrm{H}), 6.86(\mathrm{~d}, J=8.2 \mathrm{~Hz}, 1 \mathrm{H}), 6.71(\mathrm{dd}, J=10.2$ and $1.9 \mathrm{~Hz}, 1 \mathrm{H}), 6.07-5.87($ complex $\mathrm{m}, 4 \mathrm{H}), 5.28(\mathrm{dd}, J=17.1 \mathrm{and} 1.7 \mathrm{~Hz}, 1 \mathrm{H}), 5.18$ 
(dd, $J=10.2$ and $2.1 \mathrm{~Hz}, 1 \mathrm{H}), 5.14-4.97$ (complex m, 5H), $3.40(\mathrm{~d}, J=6.8 \mathrm{~Hz}, 2 \mathrm{H}), 3.31(\mathrm{~d}, J=7.1 \mathrm{~Hz}, 2 \mathrm{H}), 2.97(\mathrm{dd}, J=14.1 \mathrm{and} 7.1 \mathrm{~Hz}, 1 \mathrm{H})$, 2.92-2.77 (complex m, 3H)

${ }^{13} \mathbf{C}$ NMR $\left[100 \mathrm{MHz},\left(\mathrm{CD}_{3}\right)_{2} \mathrm{CO}\right] \delta$ see Table $\mathbf{S 1}$

IR $(\mathrm{KBr}) v_{\max } 3386,3081,2974,2913,1682,1642,1499,1415,1220,997,915 \mathrm{~cm}^{-1}$

MS (ESI, +ve) $m / z 421\left[(\mathrm{M}+\mathrm{Na})^{+}, 100 \%\right]$

HRMS (ESI, +ve) $(\mathrm{M}+\mathrm{Na})^{+}$calcd for $\mathrm{C}_{27} \mathrm{H}_{26} \mathrm{NaO}_{3} 421.1780$, found 421.1781 . 
Table S1: Comparison of the ${ }^{13}$ C NMR Data Recorded for

Compound ( \pm )-1 Obtained by the Present Route with Those

Reported by Wang ${ }^{6}$

\begin{tabular}{|c|c|c|}
\hline $\begin{array}{c}{ }^{13} \mathrm{C} \text { NMR Data for } \\
\text { Compound }( \pm)-1 \\
\left(\delta_{C}\right)^{\mathrm{a}}\end{array}$ & $\begin{array}{c}{ }^{13} \text { C NMR Data from } \\
\text { Wang } \\
\left(\delta_{C}\right)^{b}\end{array}$ & $\Delta \delta$ \\
\hline 195.1 & 195.1 & +0.1 \\
\hline 155.3 & 155.2 & +0.1 \\
\hline 153.6 & 153.5 & 0 \\
\hline 149.5 & 149.5 & +0.1 \\
\hline 139.1 & 139.0 & +0.1 \\
\hline 139.0 & 138.9 & +0.1 \\
\hline 134.3 & 134.2 & +0.1 \\
\hline 133.9 & 133.8 & +0.1 \\
\hline 132.8 & 132.7 & +0.1 \\
\hline 132.0 & 131.9 & +0.1 \\
\hline 131.9 & 131.8 & +0.1 \\
\hline 131.7 & 131.6 & +0.1 \\
\hline 129.7 & 129.7 & 0 \\
\hline 127.6 & 127.5 & +0.1 \\
\hline 125.0 & 124.9 & +0.1 \\
\hline 123.3 & 123.3 & 0 \\
\hline 122.3 & 122.2 & +0.1 \\
\hline 119.7 & 119.6 & +0.1 \\
\hline 117.3 & 117.2 & +0.1 \\
\hline 115.8 & 115.7 & +0.1 \\
\hline 115.5 & 115.5 & 0 \\
\hline 85.8 & 85.7 & +0.1 \\
\hline 49.6 & 49.5 & +0.1 \\
\hline 40.8 & 40.7 & +0.1 \\
\hline 40.4 & 40.3 & +0.1 \\
\hline 39.9 & 39.9 & 0 \\
\hline 39.4 & 39.3 & +0.1 \\
\hline
\end{tabular}

${ }^{\mathrm{a}}$ spectrum recorded in $\mathrm{CDCl}_{3}$ at $200 \mathrm{MHz}$; data obtained from reference 4, spectrum recorded in $\mathrm{CDCl}_{3}$ at $125 \mathrm{MHz}$. 


\section{Crystallographic Study}

\section{Crystallographic Data for Compound 27}

$\mathrm{C}_{27} \mathrm{H}_{28} \mathrm{O}_{3}, M=400.47, T=150 \mathrm{~K}$, triclinic, space group $\mathrm{P} \overline{1}, Z=4, a=9.9759(2) \AA, b=13.6806(3) \AA, c=17.6508(4) \AA ; \alpha=109.1900(19)^{\circ}, \beta$ $=96.4682(17)^{\circ}, \gamma=98.2575(16)^{\circ} ; V=2218.30(5) \AA^{3}, D_{x}=1.199 \mathrm{~g} \mathrm{~cm}^{-3}, 8471$ unique data $\left(2 \Theta_{\max }=144.0^{\circ}\right), R=0.052$ [for 7181 reflections with $I>2.0 \sigma(I)] ; R w=0.143$ (all data), $S=0.99$.

\section{Structure Determination}

Images were measured on a diffractometer ( $\mathrm{Cu} \mathrm{K \alpha}$, mirror monochromator, $\lambda=1.54184 \AA$ ) fitted with an area detector and data extracted using the CrysAlis package. ${ }^{7}$ Structure solution was by direct methods (SIR92). ${ }^{8}$ The structure of compound 27 was refined using the CRYSTALS program package. ${ }^{9}$ Atomic coordinates, bond lengths and angles, and displacement parameters for compound 27 have been deposited at the Cambridge Crystallographic Data Centre (CCDC no. 1482403). These data can be obtained free-of-charge via www.ccdc.cam.ac.uk/data_request/cif, by emailing data_request@ccdc.cam.ac.uk, or by contacting The Cambridge Crystallographic Data Centre, 12 Union Road, Cambridge CB2 1EZ, UK; fax: $+\overline{4} 41223336033$. 


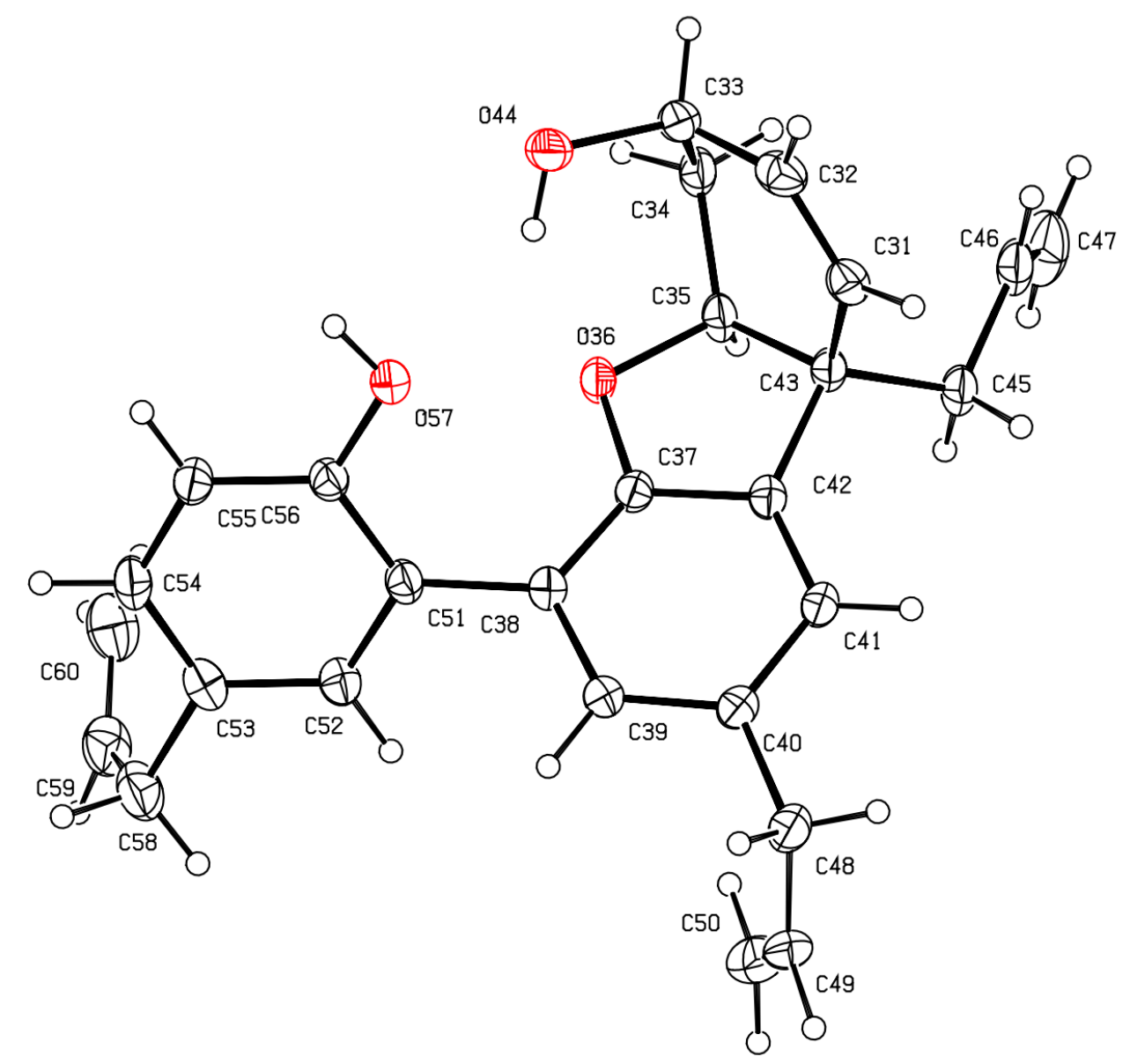

Figure S1: Structure of one selected molecule of compound 27 (CCDC 1482403) in the asymmetric unit with labeling of selected atoms. Anisotropic displacement ellipsoids show $30 \%$ probability levels. Hydrogen atoms are drawn as circles with small radii. 


\section{References}

1. Still, W. C.; Kahn, M.; Mitra, A. J. Org. Chem., 1978, 43, 2923.

2. Pangborn, A. B.; Giardello, M. A.; Grubbs, R. H.; Rosen, R. K.; Timmers, F. J. Organometallics, 1996, $15,1518$.

3. Nugent, J.; Matoušová, E.; Banwell, M. G. Eur. J. Org. Chem., 2015, 3771.

4. Whelligan, D. K.; Thomson, D. W.; Taylor, D.; Hoelder, S. J. Org. Chem. 2010, 75, 11.

5. Berliner, M. A.; Belecki, K. J. Org. Chem. 2005, 70, 9618.

6. Homsi, F.; Robin, S.; Rousseau, G. Org. Synth., 2000, 77, 127.

7. CrysAlis PRO Version 1.171.37.35h (release 09-02-2015 CrysAlis171.NET) (compiled Feb 9 2015,16:26:32) Agilent Technologies: Oxfordshire, UK.

8. SIR92. Altomare, A.; Cascarano, G.; Giacovazzo, C.; Guagliardi, A.; Burla, M. C.; Polidori, G.; Camalli, M. J. Appl. Crystallogr., 1994, 27, 435 .

9. Betteridge, P. W.; Carruthers, J. R.; Cooper, R. I.; Prout, K.; Watkin, D. J. J. Appl. Crystallogr., 2003, $36,1487$. 


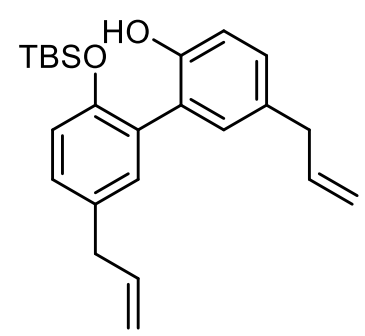

$400 \mathrm{MHz}{ }^{1} \mathrm{H}$ NMR Spectrum of Compound 8

(recorded in $\mathrm{CDCl}_{3}$ )
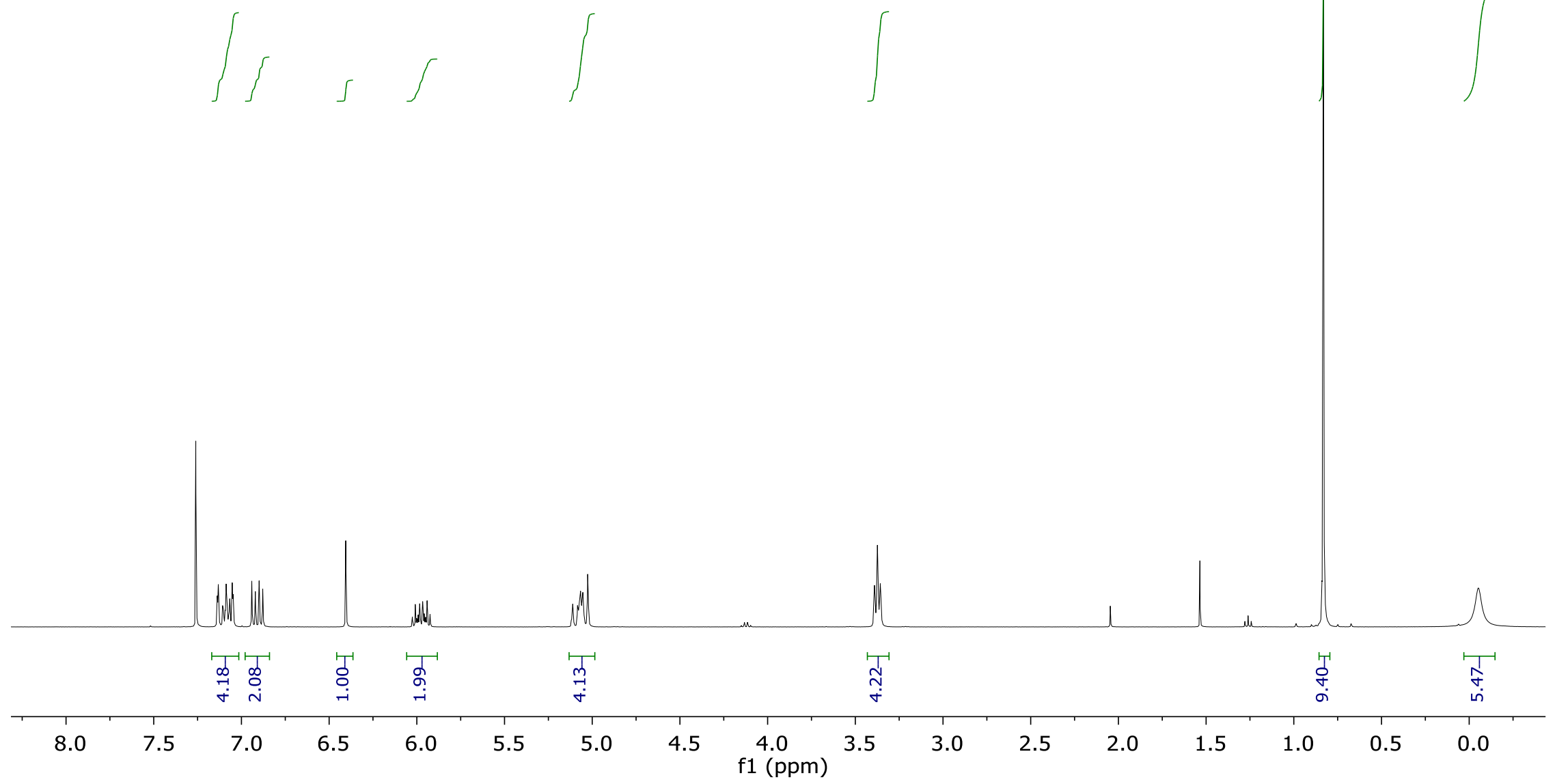


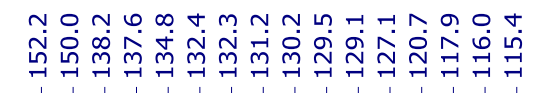

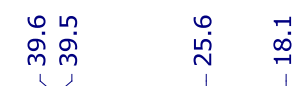

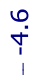

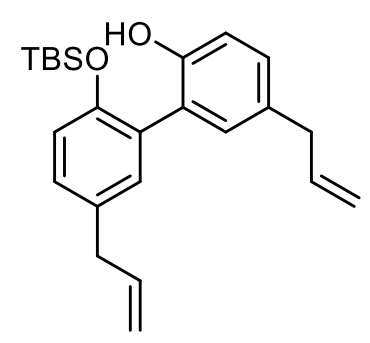

$100 \mathrm{MHz}{ }^{13} \mathrm{C}$ NMR Spectrum of Compound 8

(recorded in $\mathrm{CDCl}_{3}$ ) 


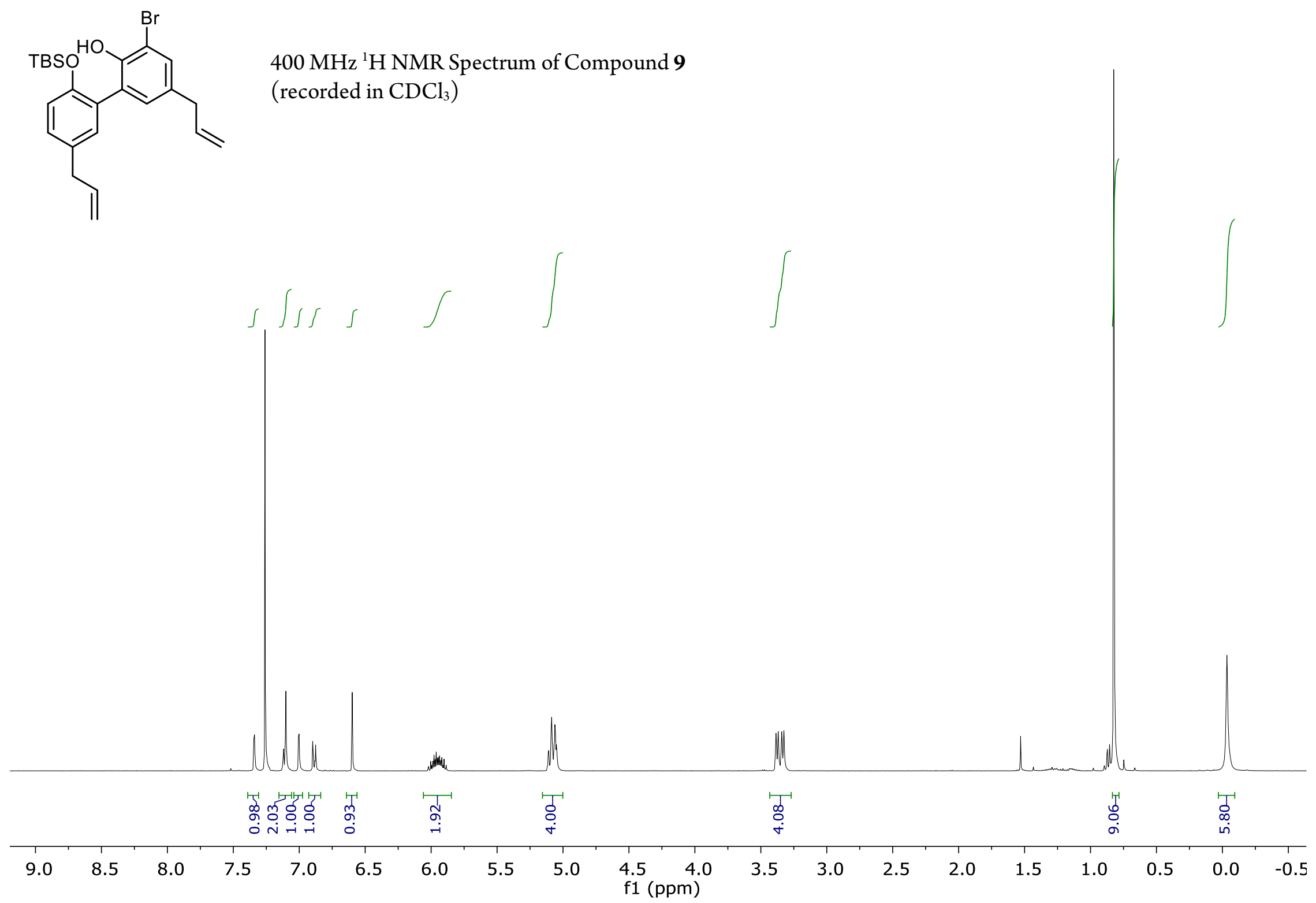




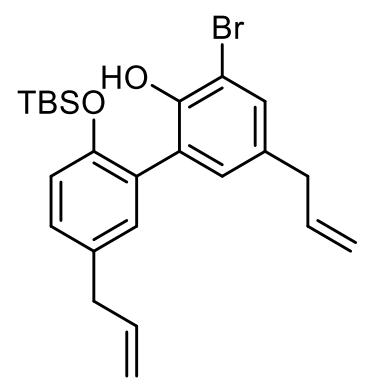

$100 \mathrm{MHz}{ }^{13} \mathrm{C}$ NMR Spectrum of Compound 9 (recorded in $\mathrm{CDCl}_{3}$ )

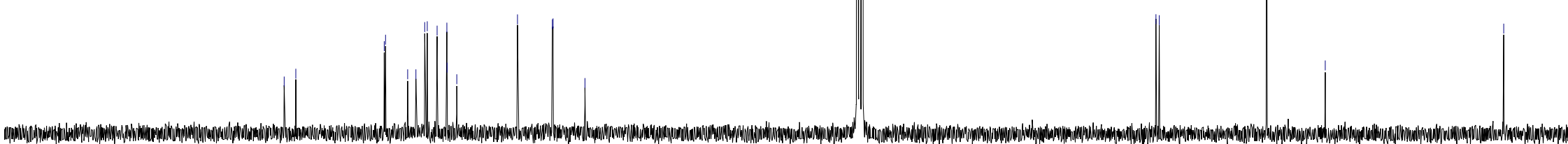




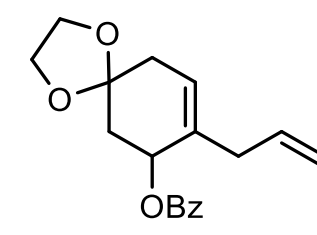

$400 \mathrm{MHz}{ }^{1} \mathrm{H}$ NMR Spectrum of Compound 13

(recorded in $\mathrm{CDCl}_{3}$ )

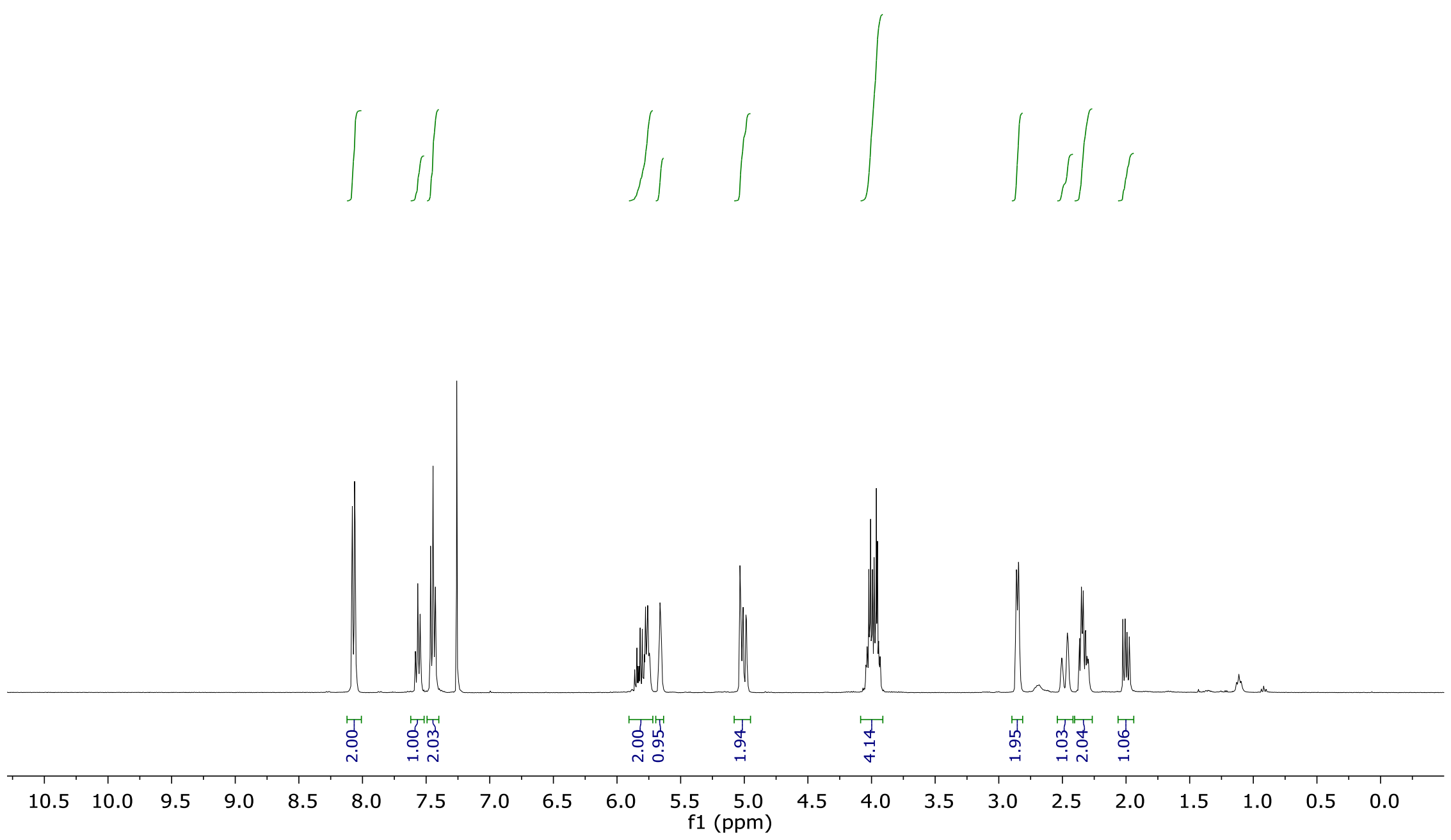




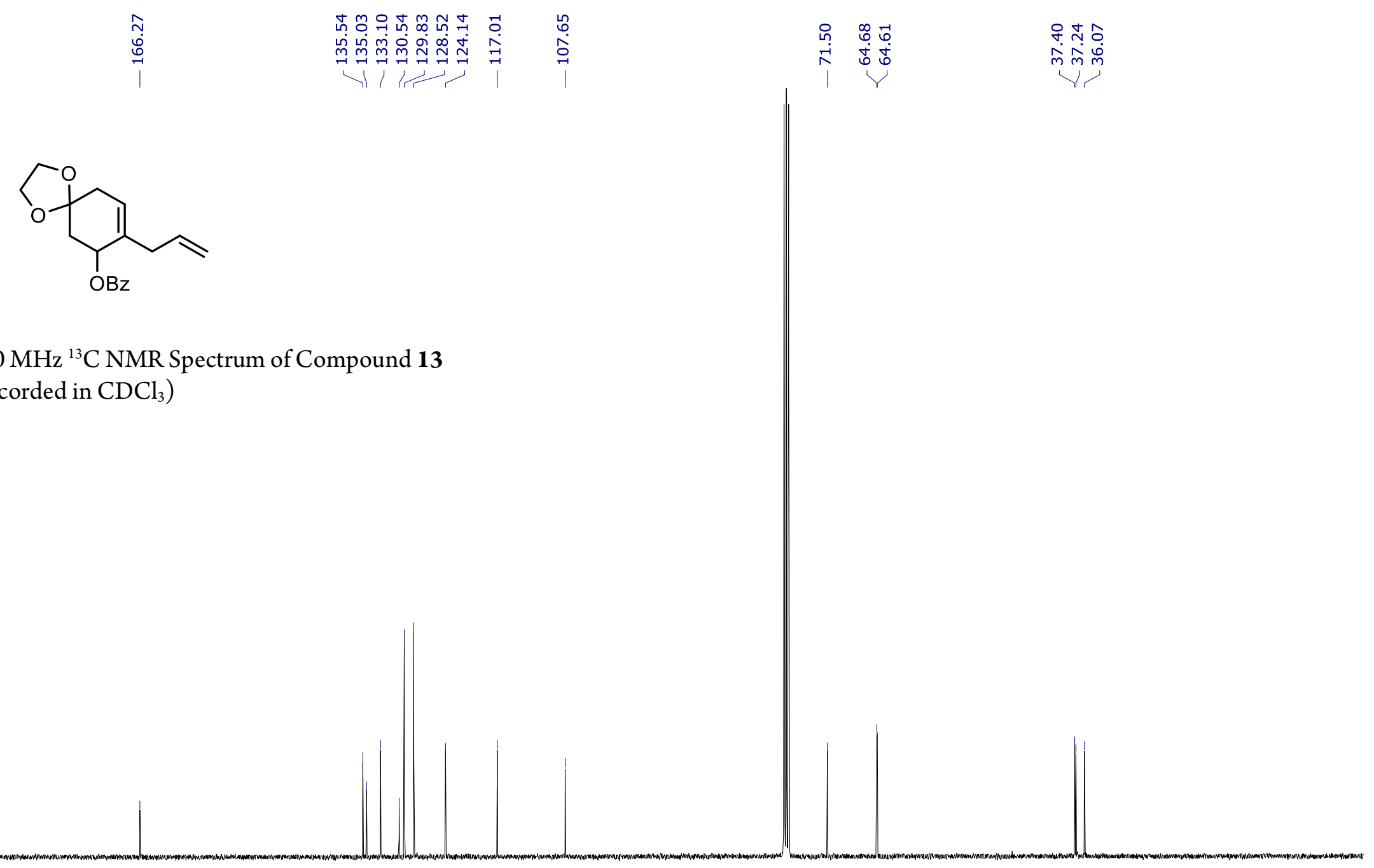



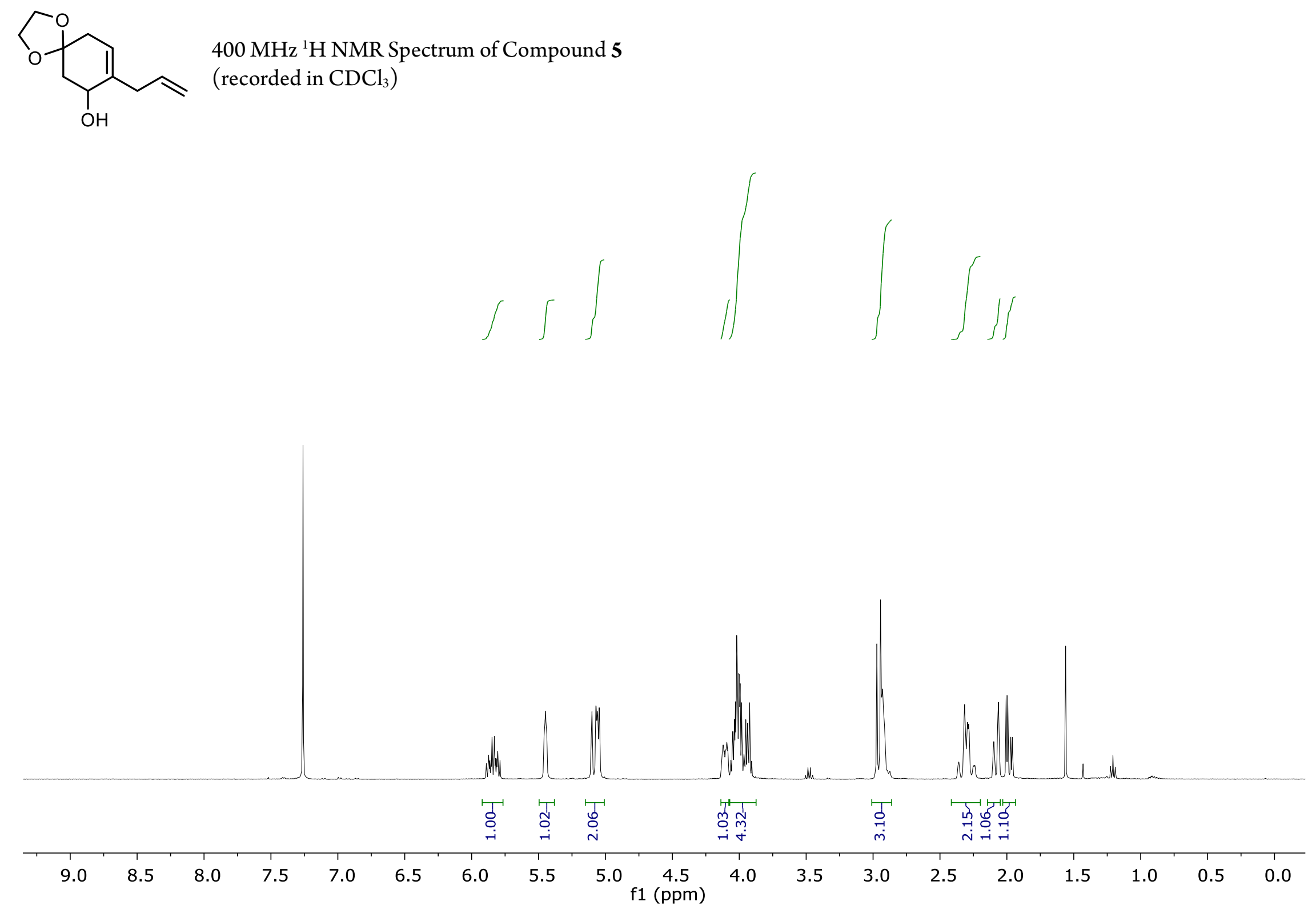

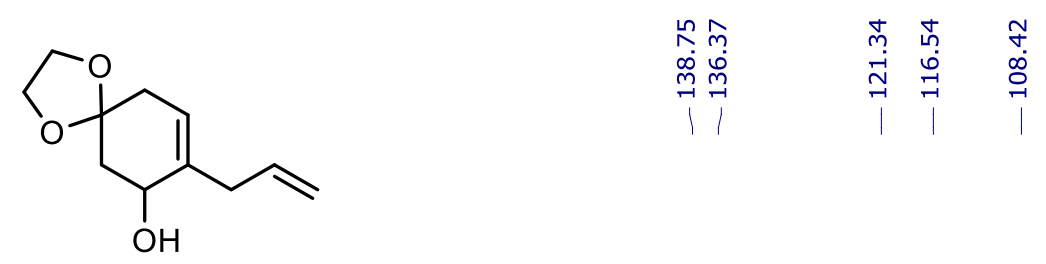

$100 \mathrm{MHz}{ }^{13} \mathrm{C}$ NMR Spectrum of Compound 5 (recorded in $\mathrm{CDCl}_{3}$ )

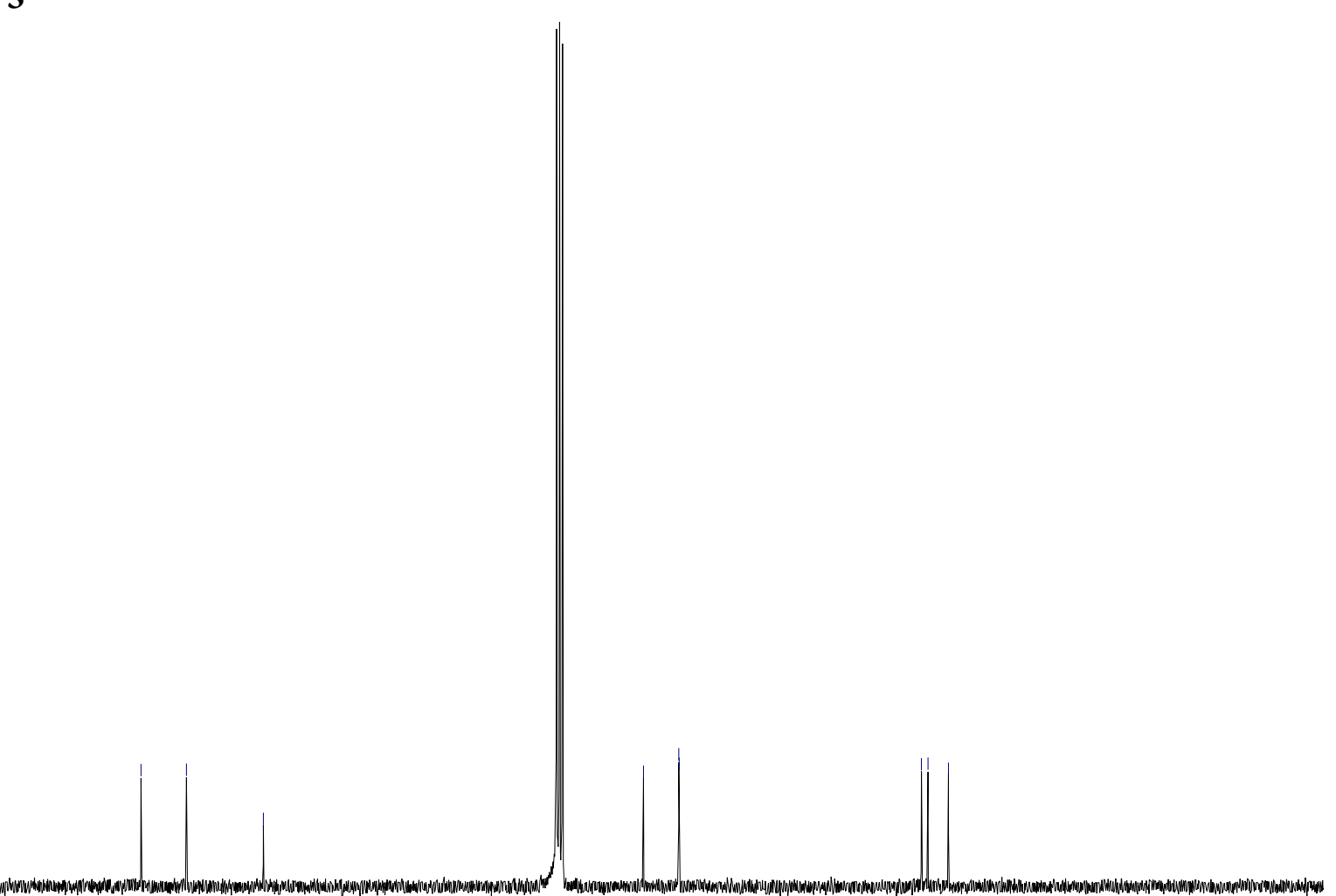




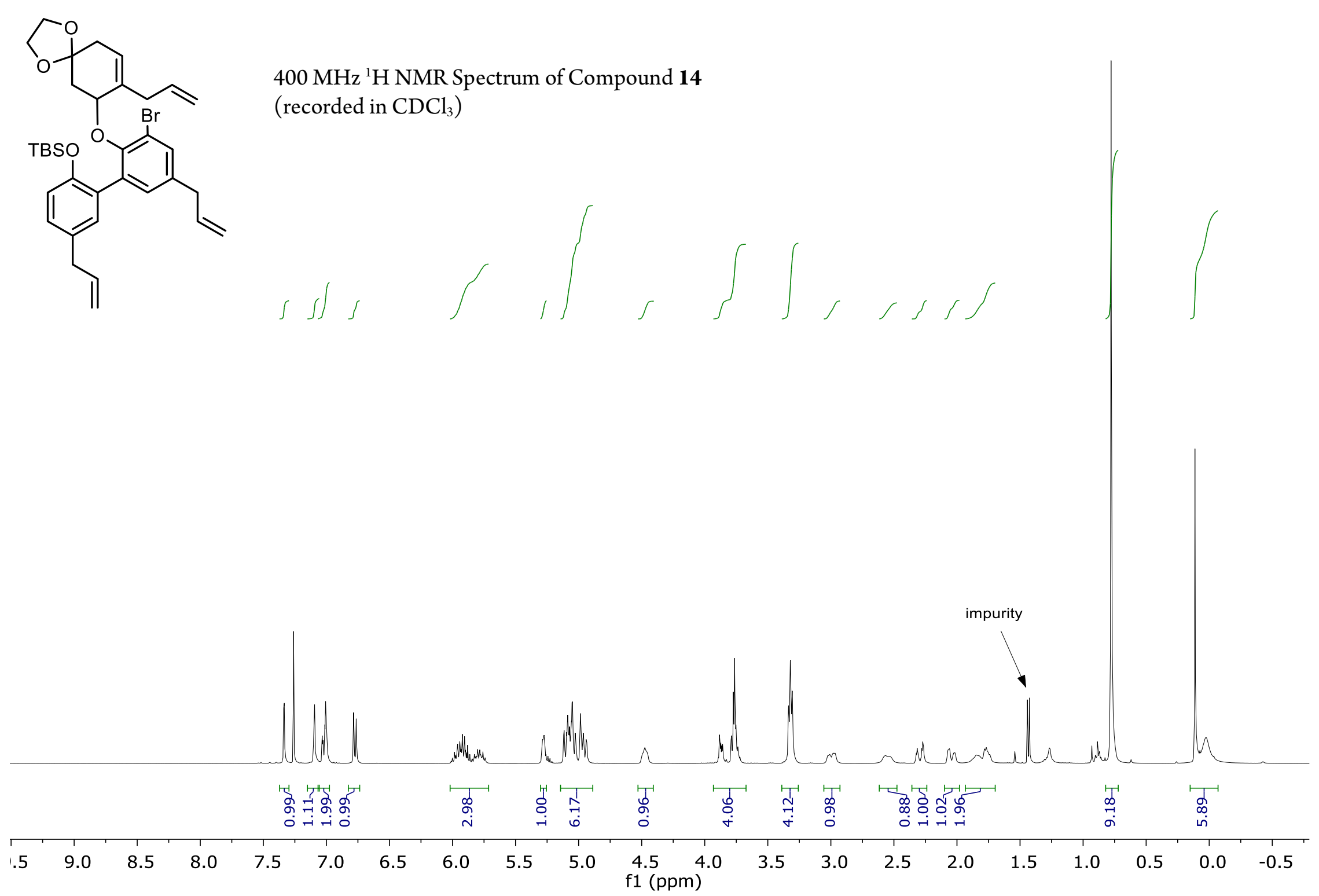




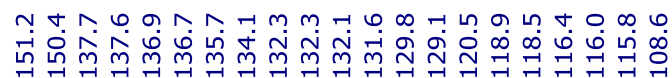
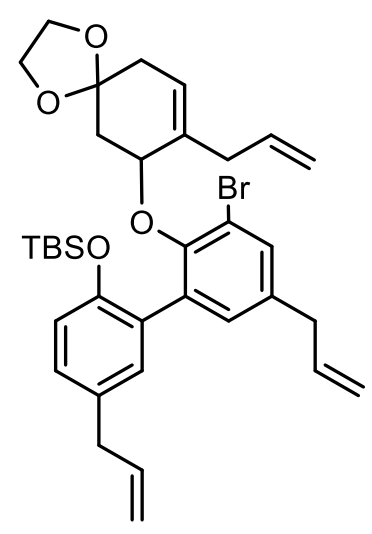

$100 \mathrm{MHz}{ }^{13} \mathrm{C}$ NMR Spectrum of Compound 14

(recorded in $\mathrm{CDCl}_{3}$ )

$*=\mathrm{EtOAc}$ 


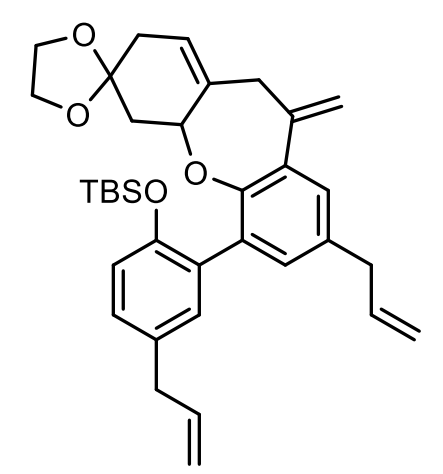

$400 \mathrm{MHz}{ }^{1} \mathrm{H}$ NMR Spectrum of Compound 15

(recorded in $\mathrm{CDCl}_{3}$ )

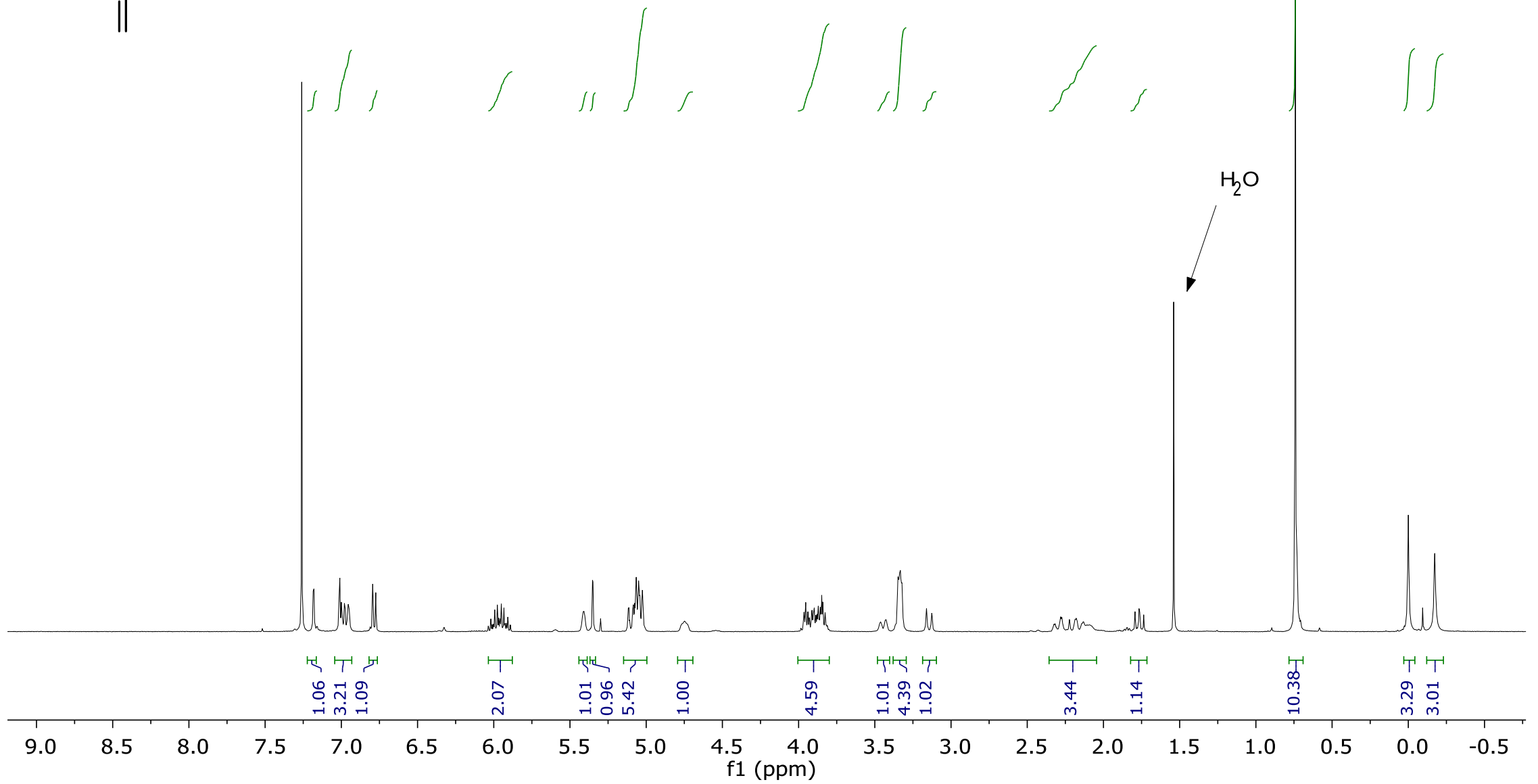



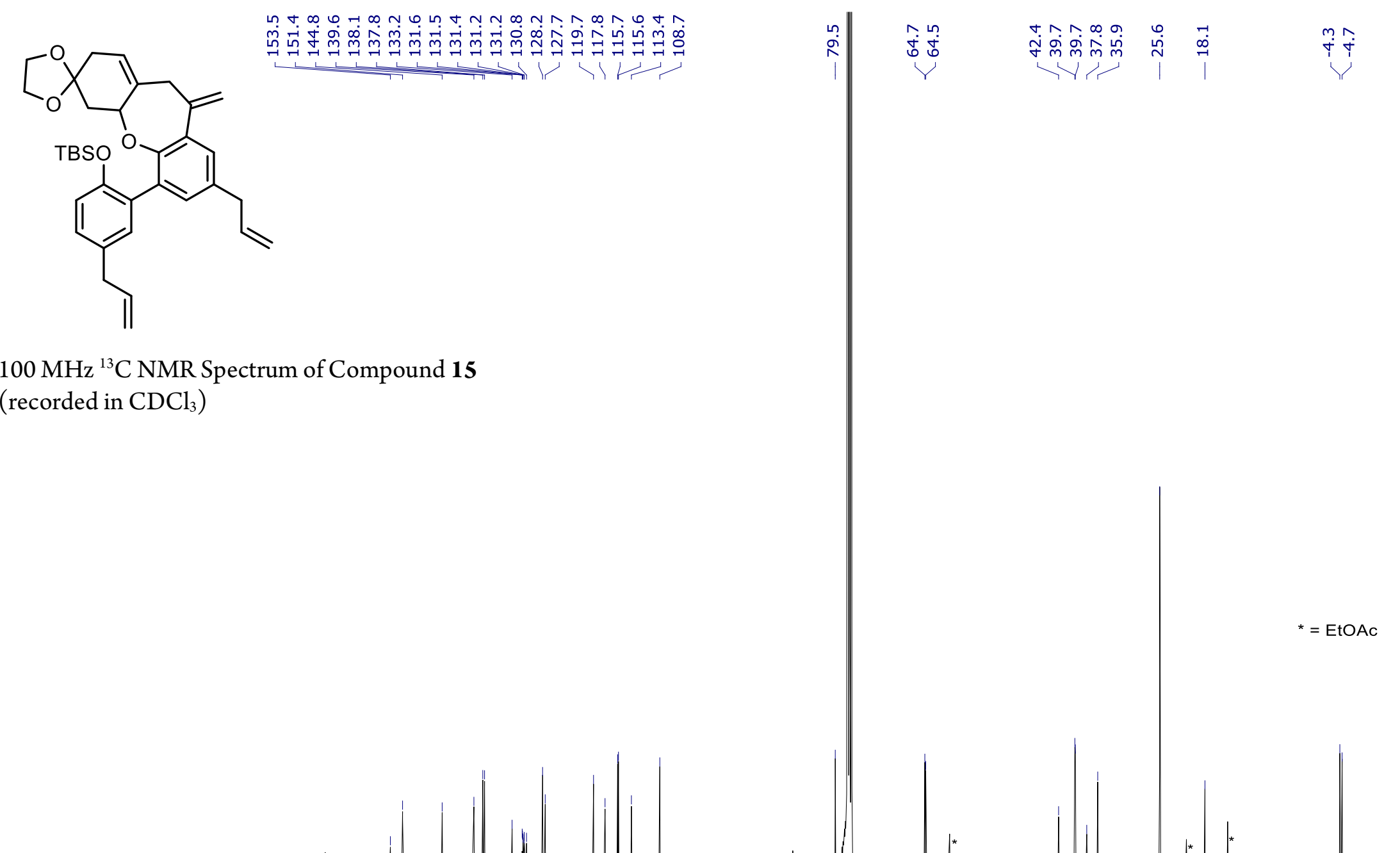

$100 \mathrm{MHz}{ }^{13} \mathrm{C}$ NMR Spectrum of Compound 15 (recorded in $\mathrm{CDCl}_{3}$ )

What

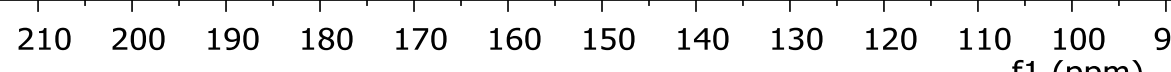

f1 (ppm) 


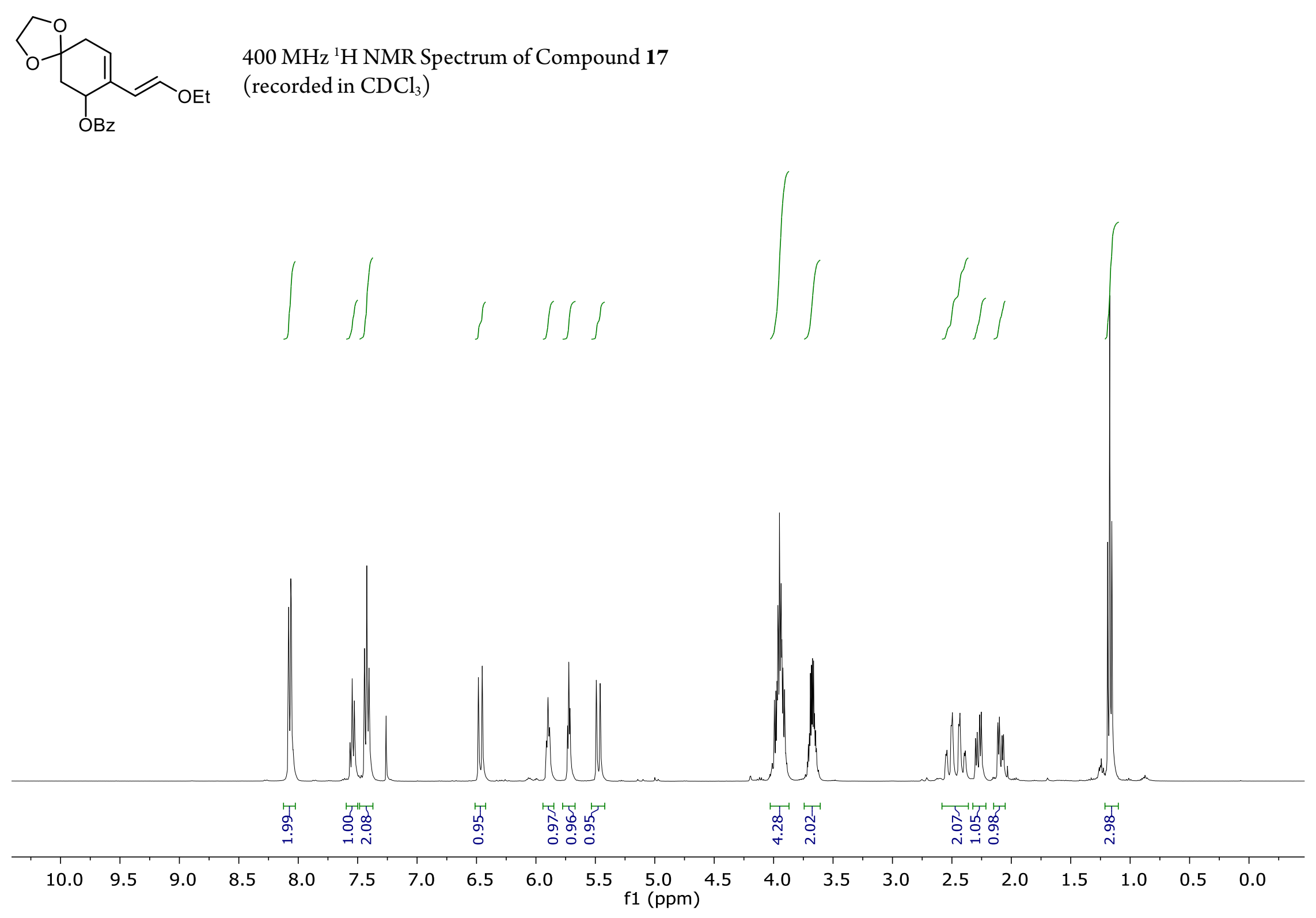




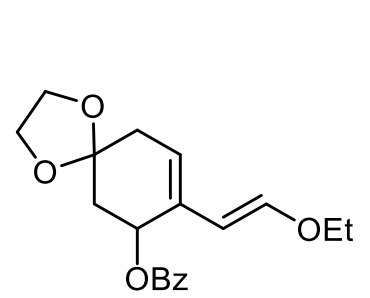

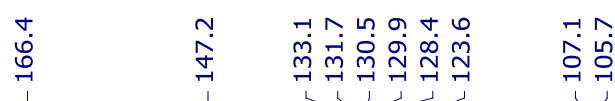

ภำ

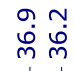

$\stackrel{\infty}{+}$

$100 \mathrm{MHz}{ }^{13} \mathrm{C}$ NMR Spectrum of Compound 17

(recorded in $\mathrm{CDCl}_{3}$ )

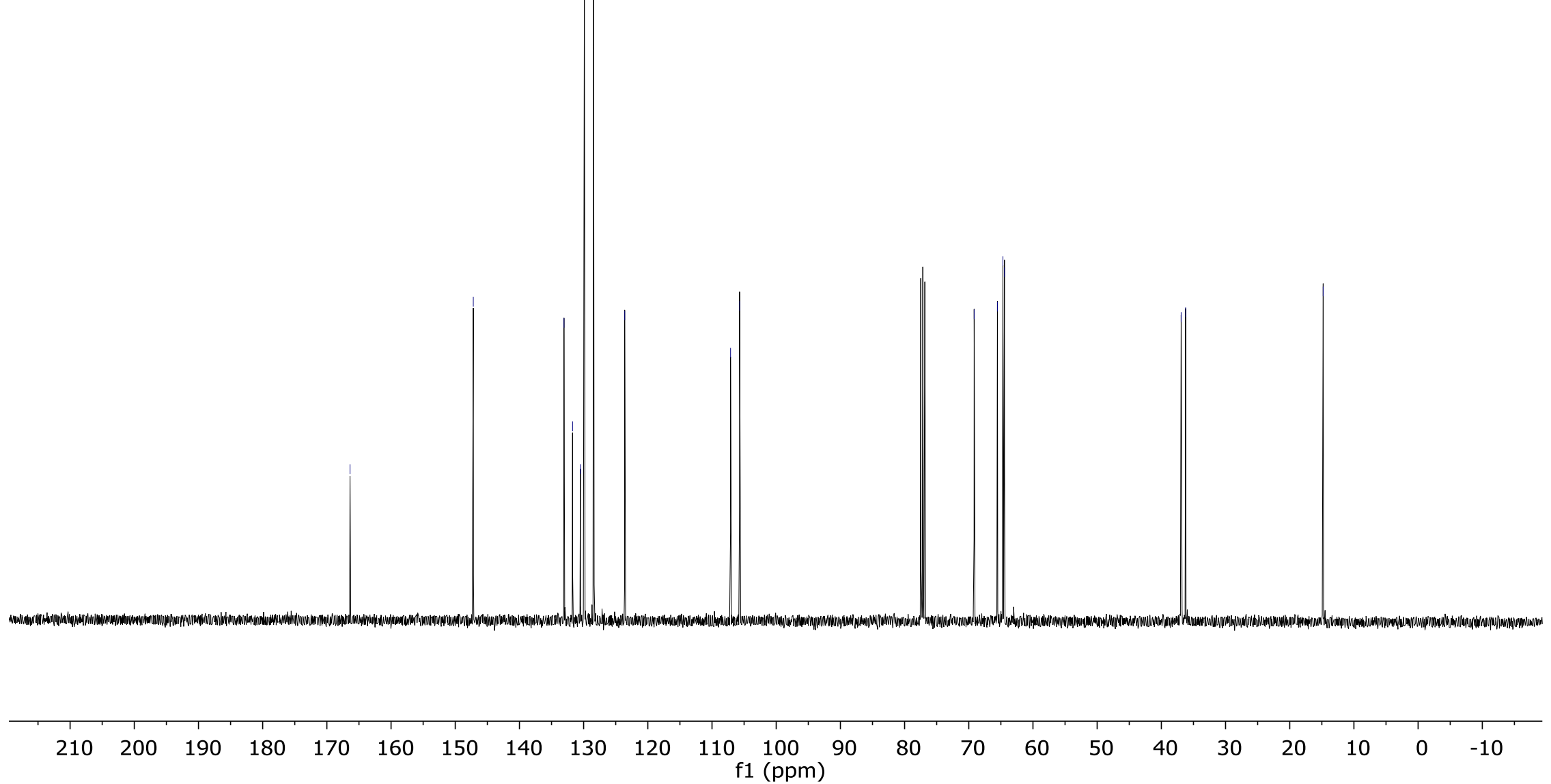



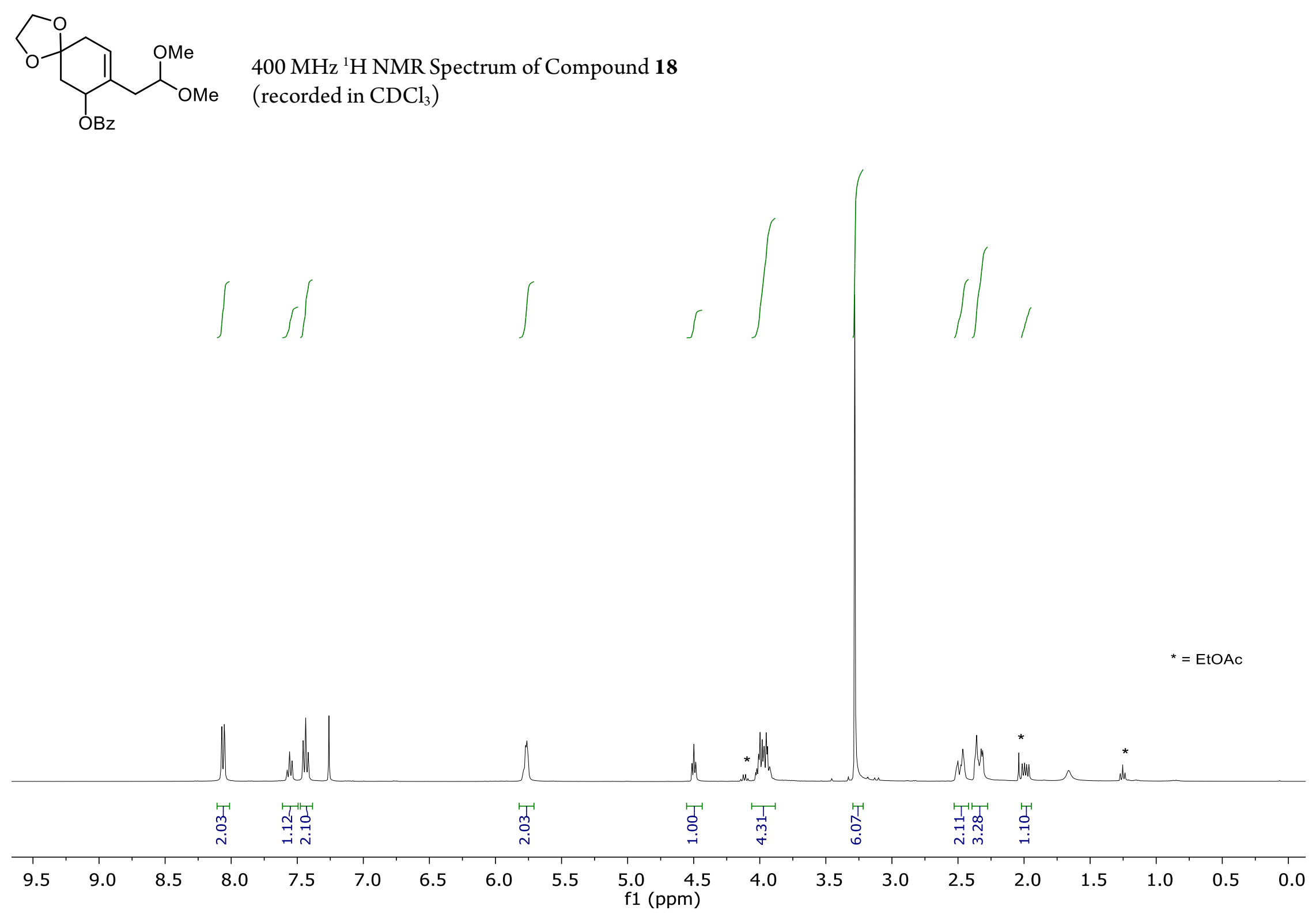


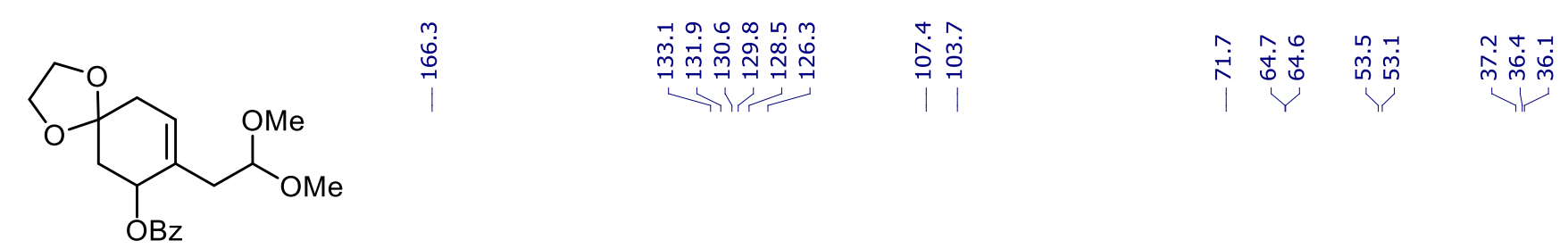

$100 \mathrm{MHz}{ }^{13} \mathrm{C}$ NMR Spectrum of Compound 18 (recorded in $\mathrm{CDCl}_{3}$ )

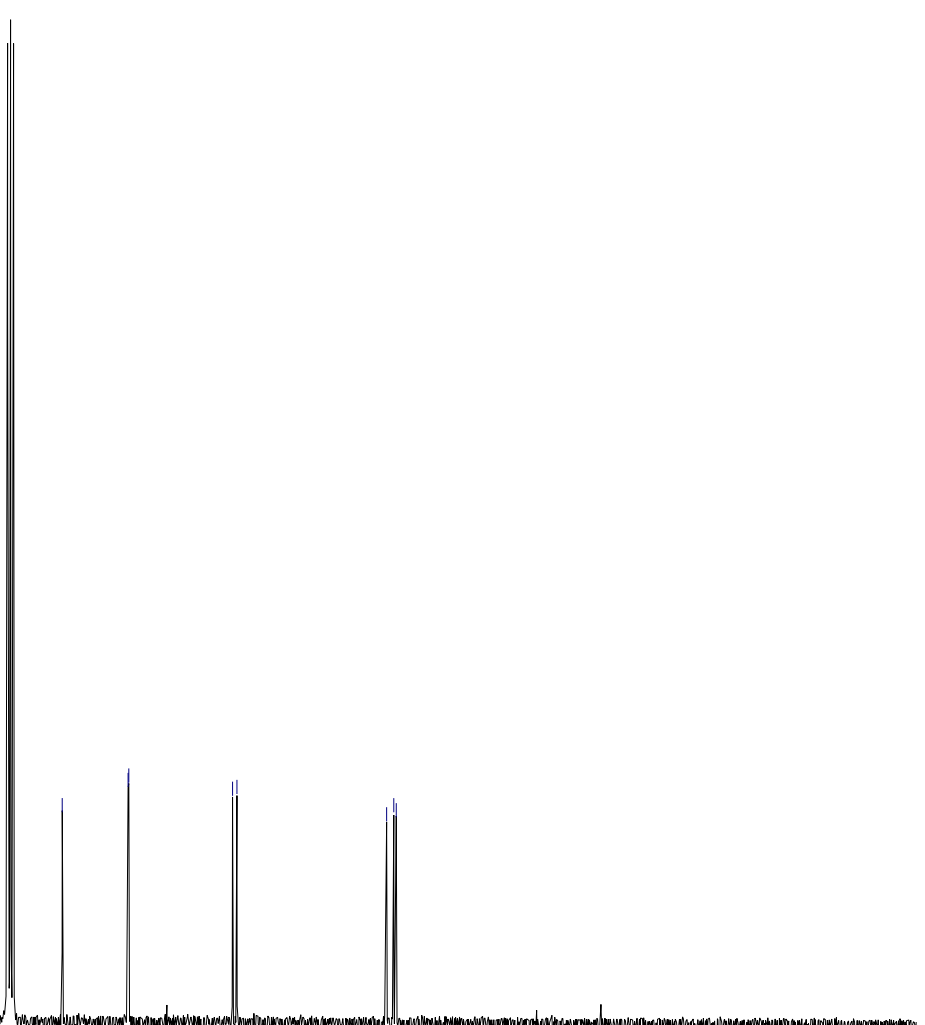

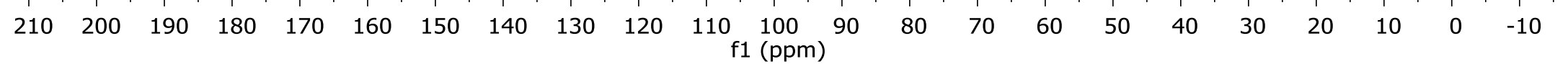




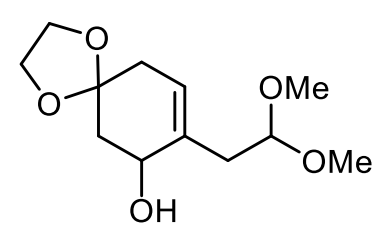

$400 \mathrm{MHz}{ }^{1} \mathrm{H}$ NMR Spectrum of Compound 19 (recorded in $\mathrm{CDCl}_{3}$ )

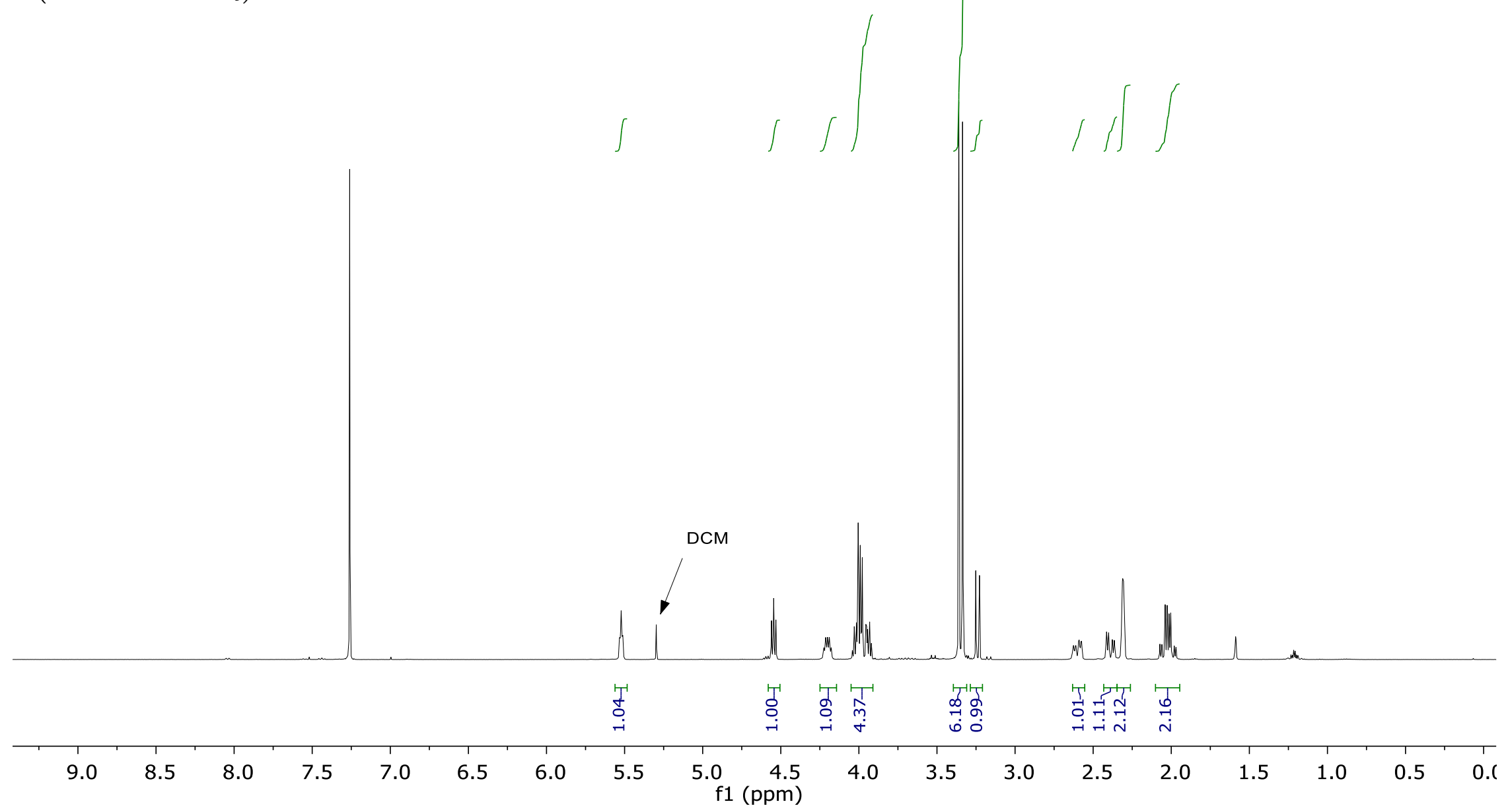



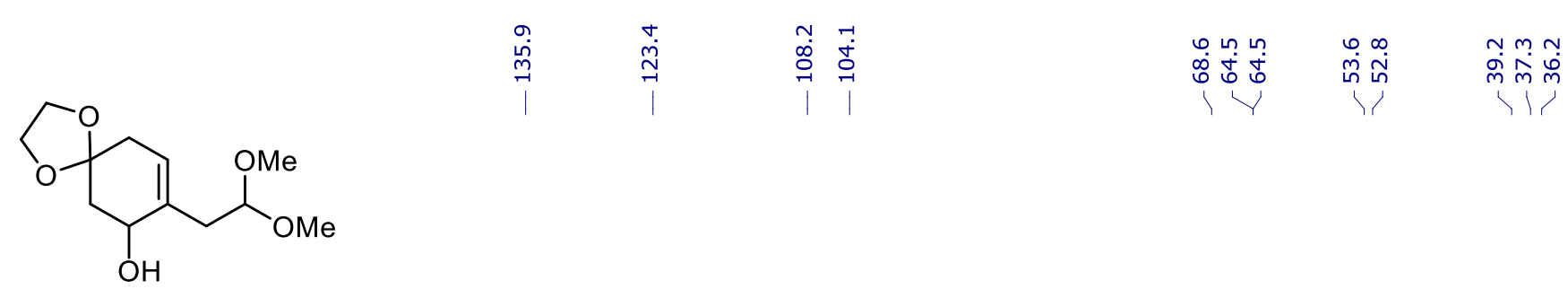

$100 \mathrm{MHz}{ }^{13} \mathrm{C}$ NMR Spectrum of Compound 19 (recorded in $\mathrm{CDCl}_{3}$ )

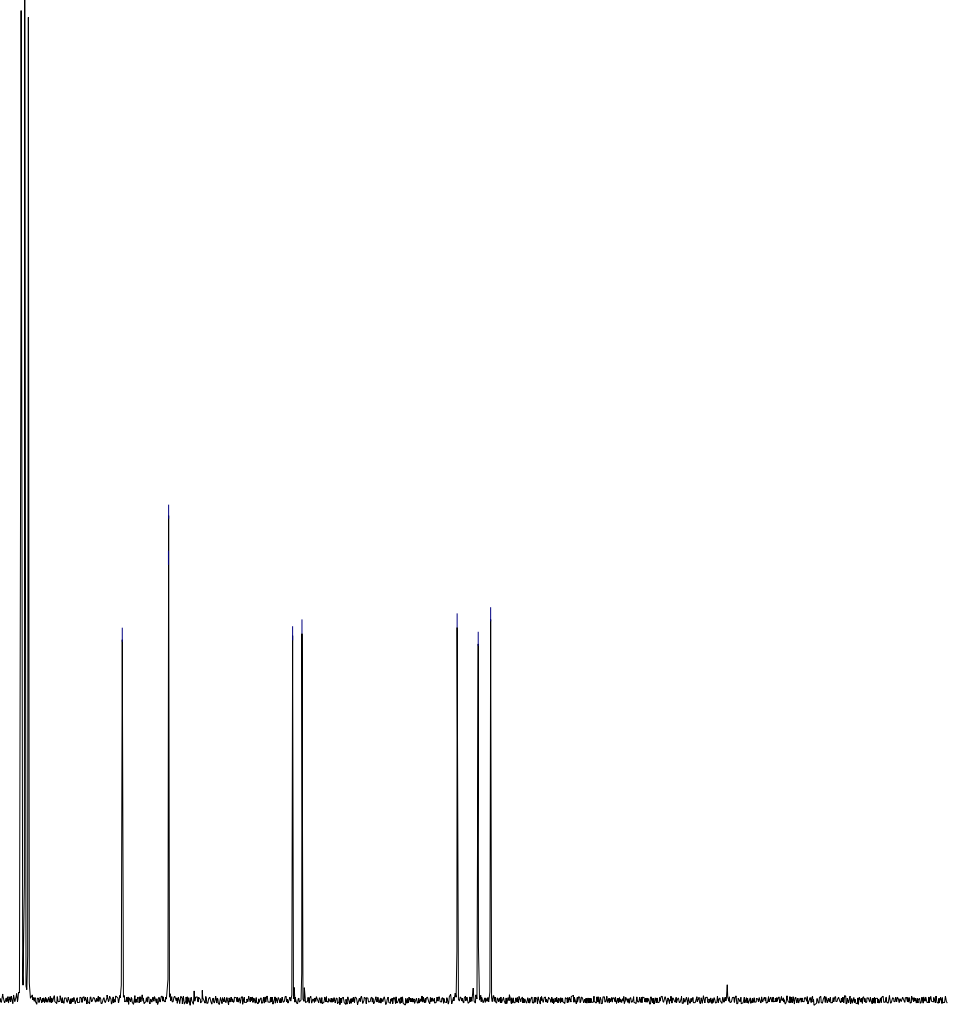




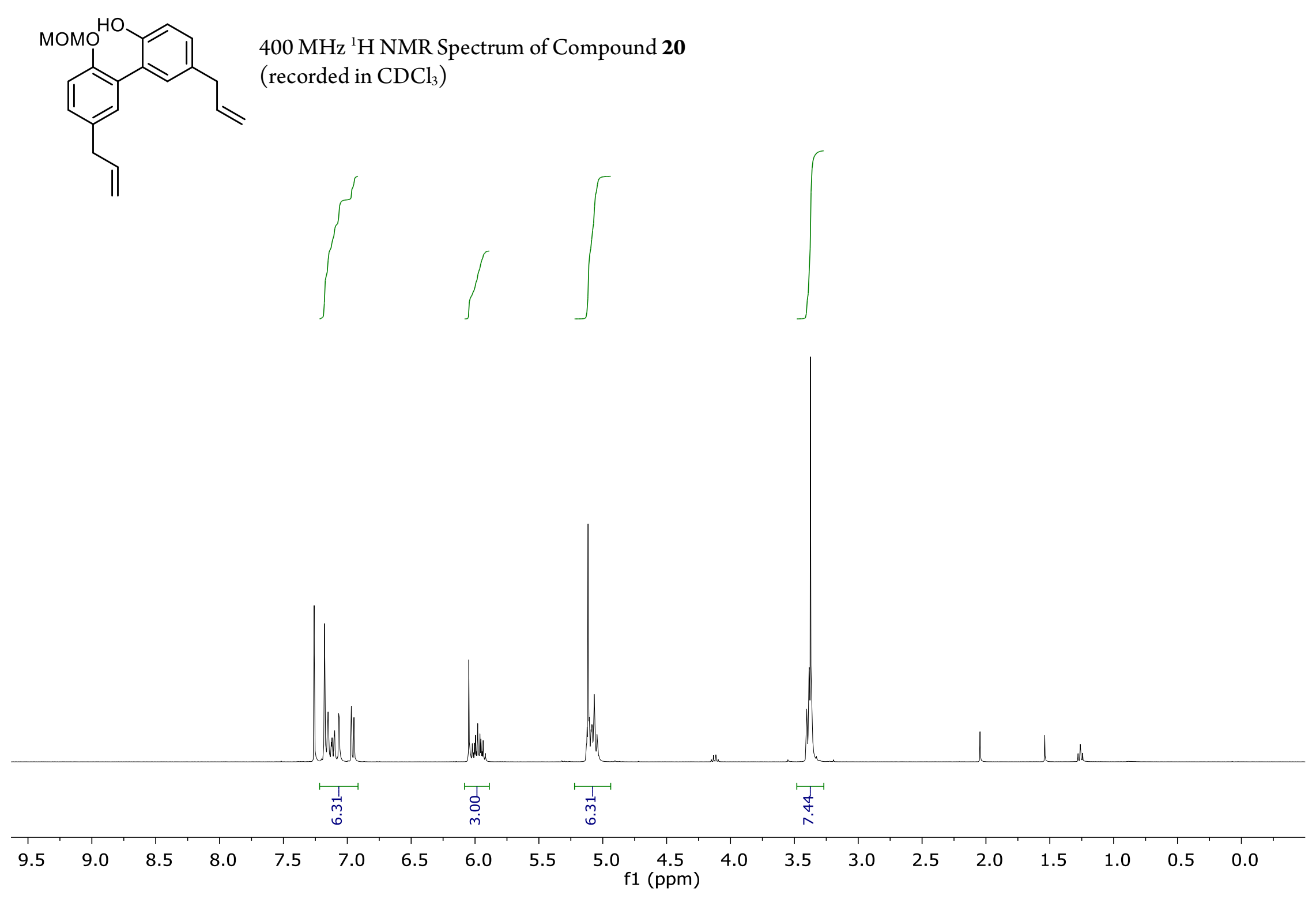




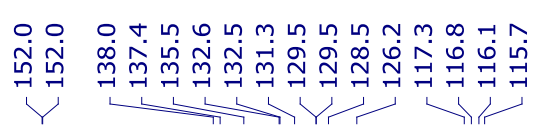

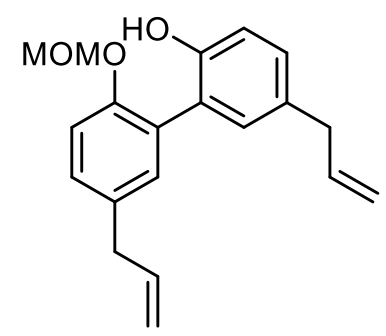

$100 \mathrm{MHz}{ }^{13} \mathrm{C}$ NMR Spectrum of Compound 20 (recorded in $\mathrm{CDCl}_{3}$ )
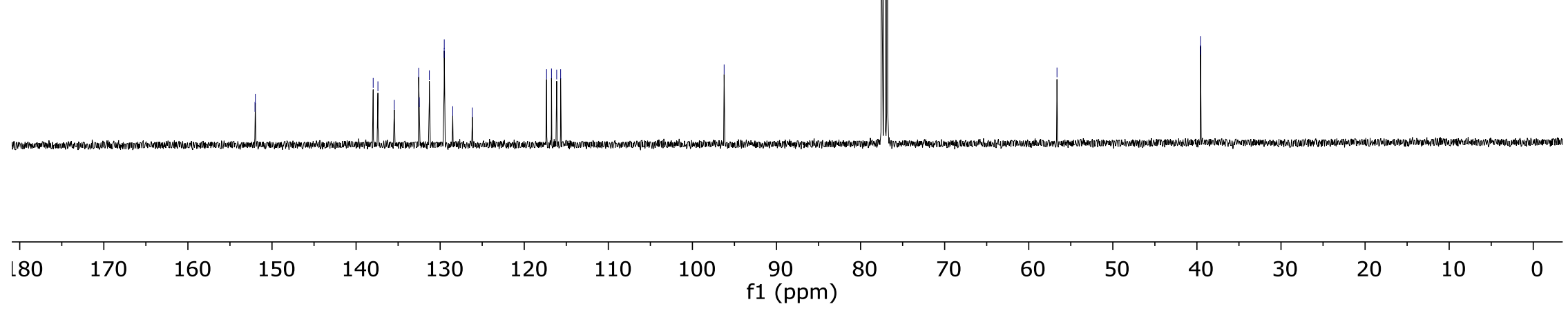

$\stackrel{\sim}{\mathscr{D}}$

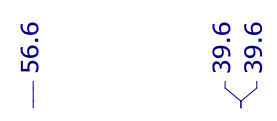

S47 


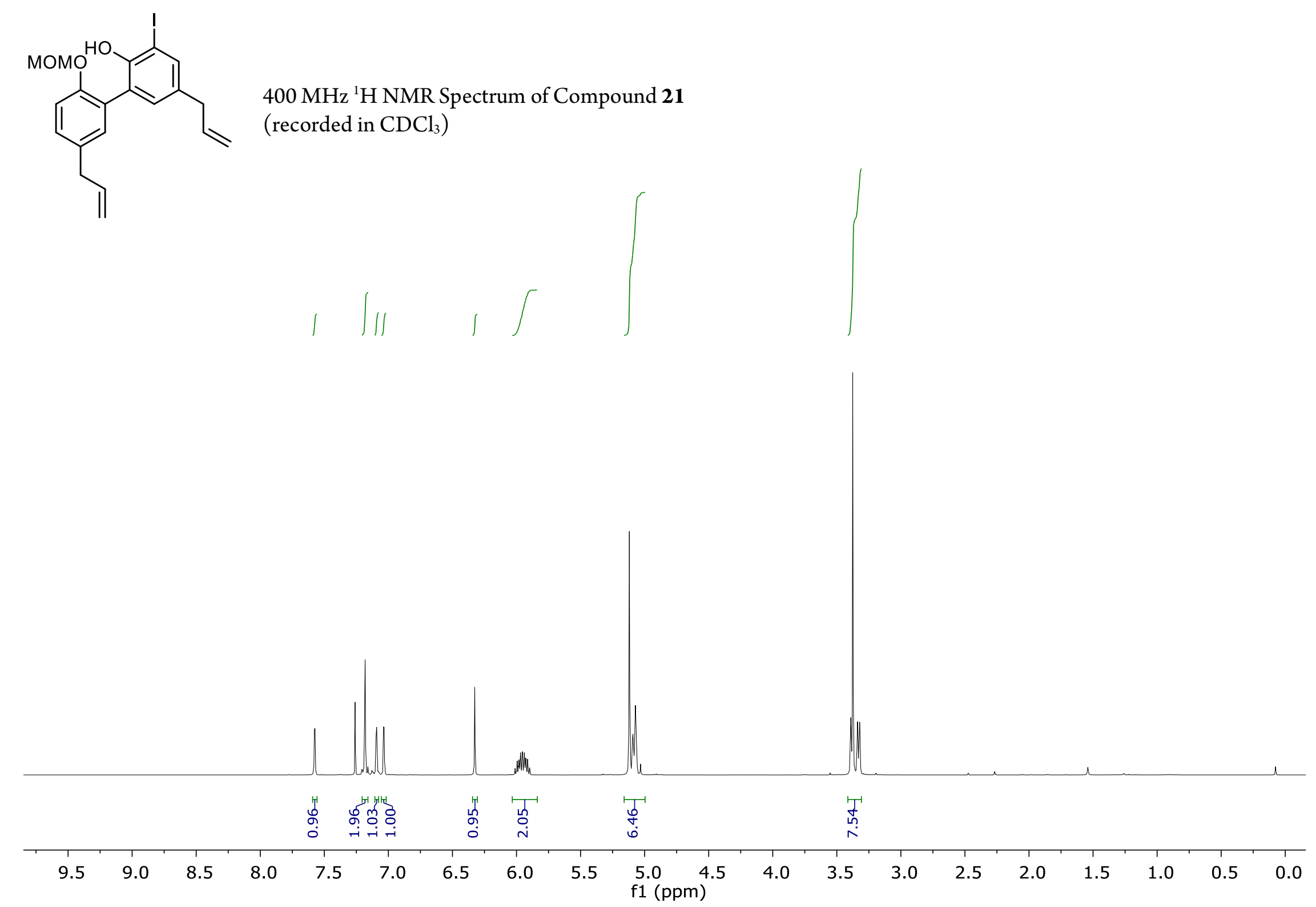




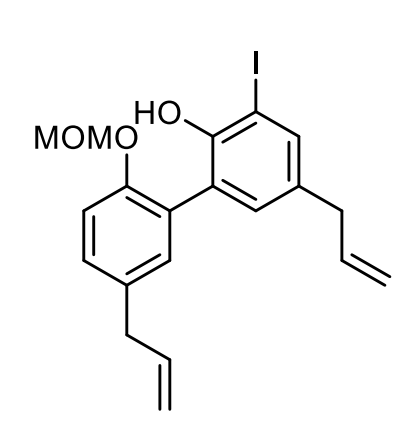

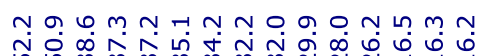

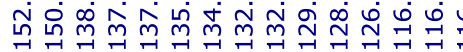

它

:

$100 \mathrm{MHz}{ }^{13} \mathrm{C}$ NMR Spectrum of Compound 21

(recorded in $\mathrm{CDCl}_{3}$ )

$\begin{array}{lllllllllllllllllllllll}210 & 200 & 190 & 180 & 170 & 160 & 150 & 140 & 130 & 120 & 110 & 100 & 90 & 80 & 70 & 60 & 50 & 40 & 30 & 20 & 10 & 0 & -10\end{array}$




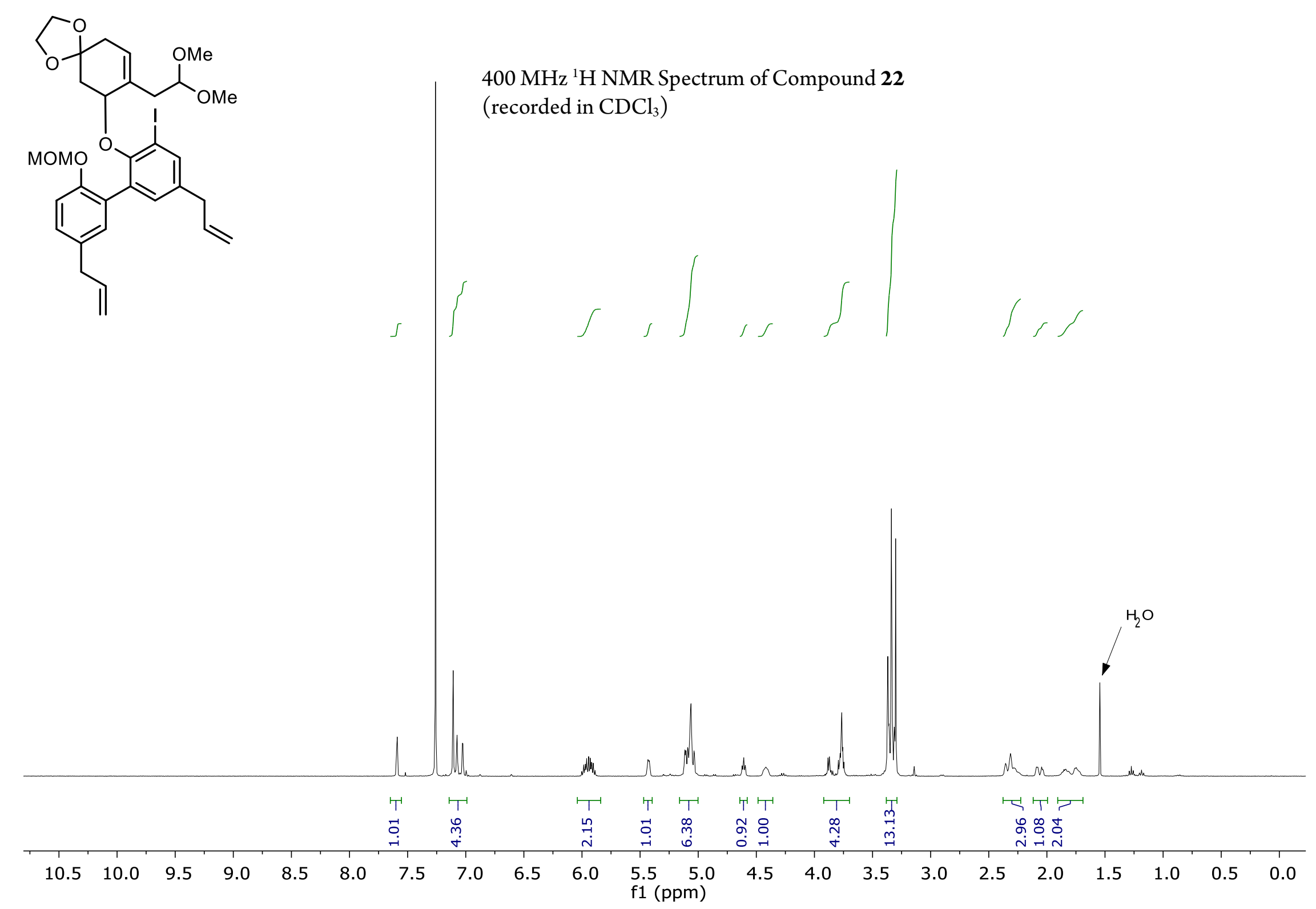




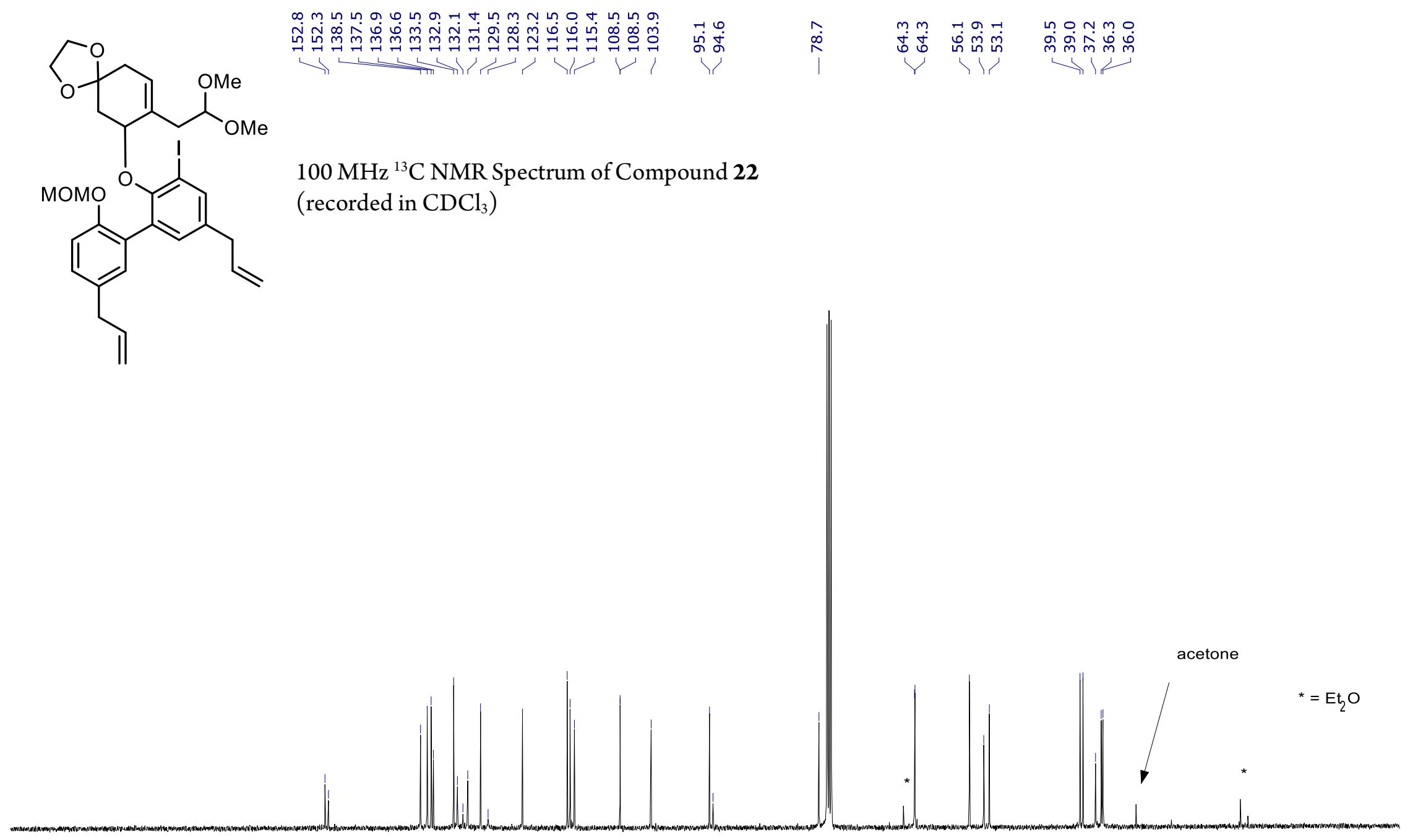

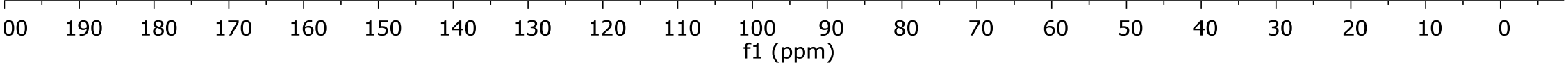




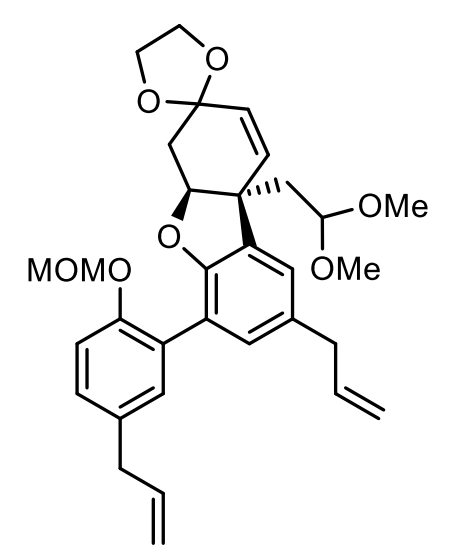

$400 \mathrm{MHz}{ }^{1} \mathrm{H}$ NMR Spectrum of Compound 23

(recorded in $\mathrm{CDCl}_{3}$ )
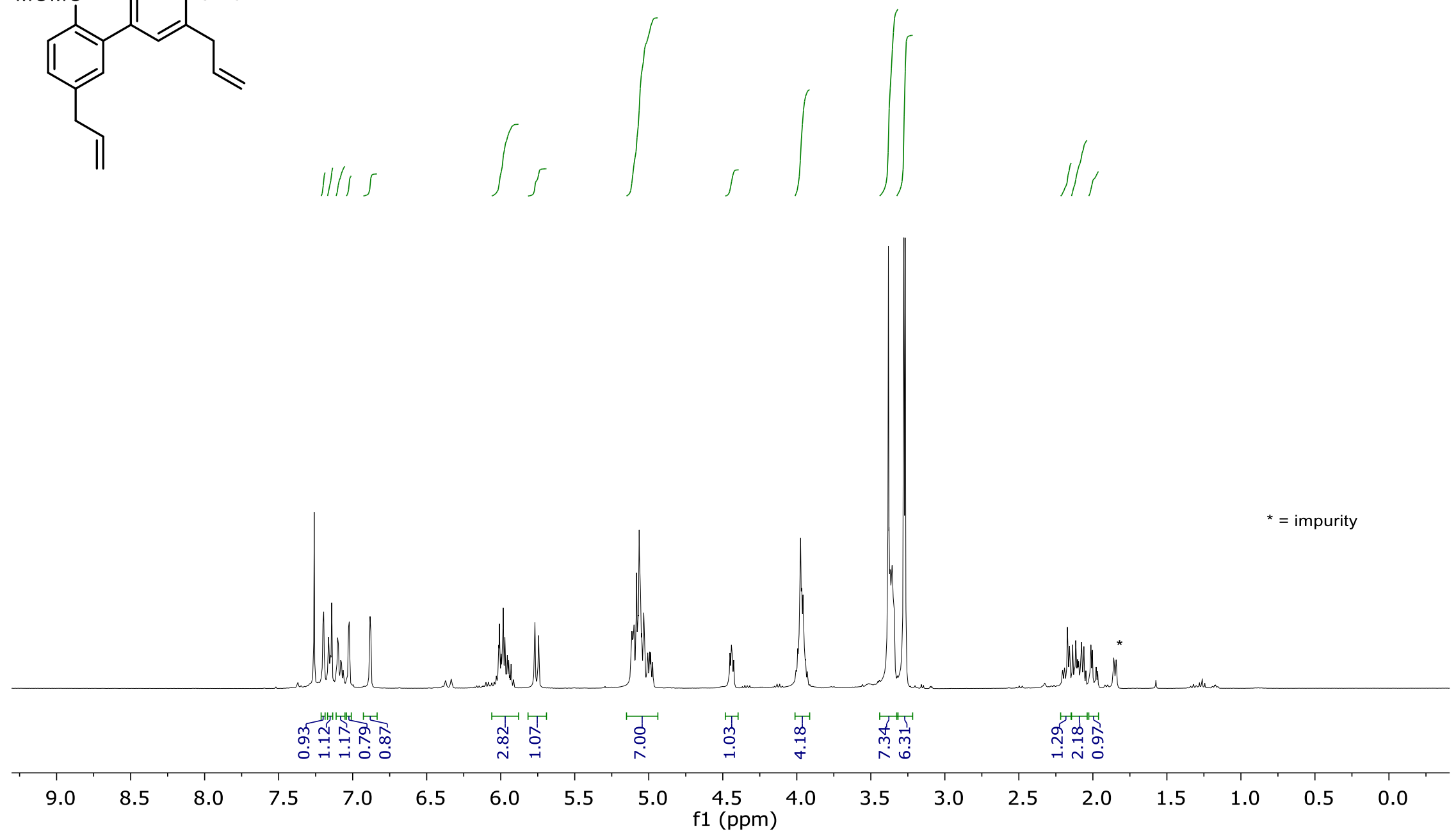

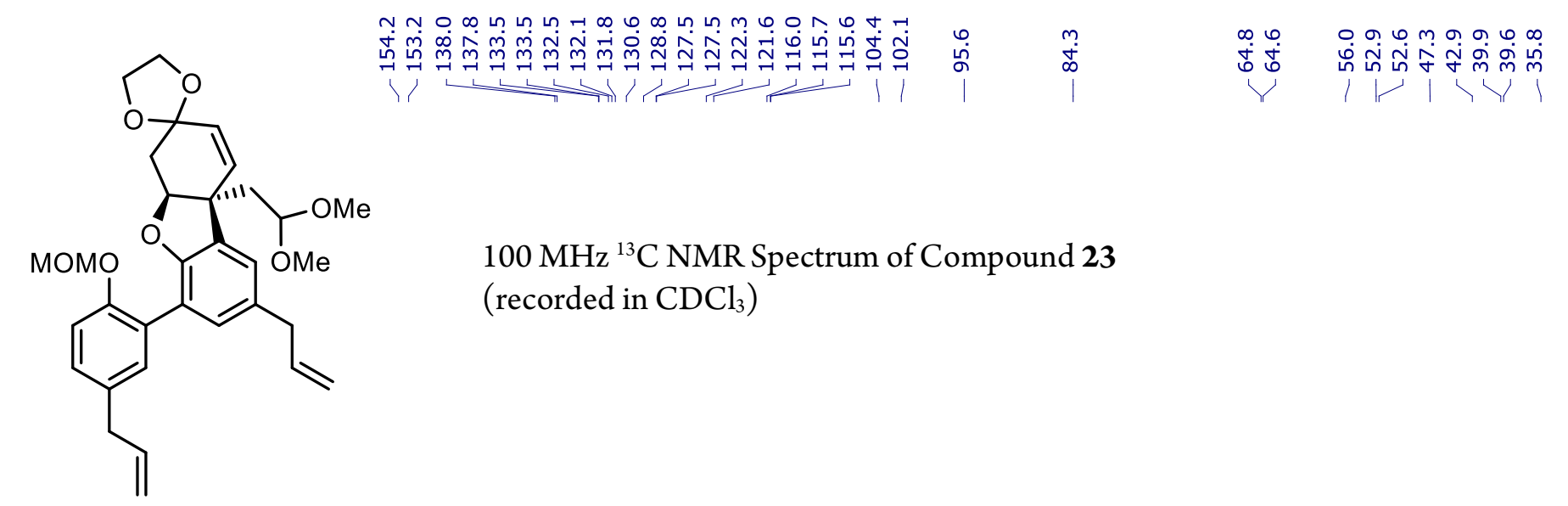

$100 \mathrm{MHz}{ }^{13} \mathrm{C}$ NMR Spectrum of Compound 23

(recorded in $\mathrm{CDCl}_{3}$ )

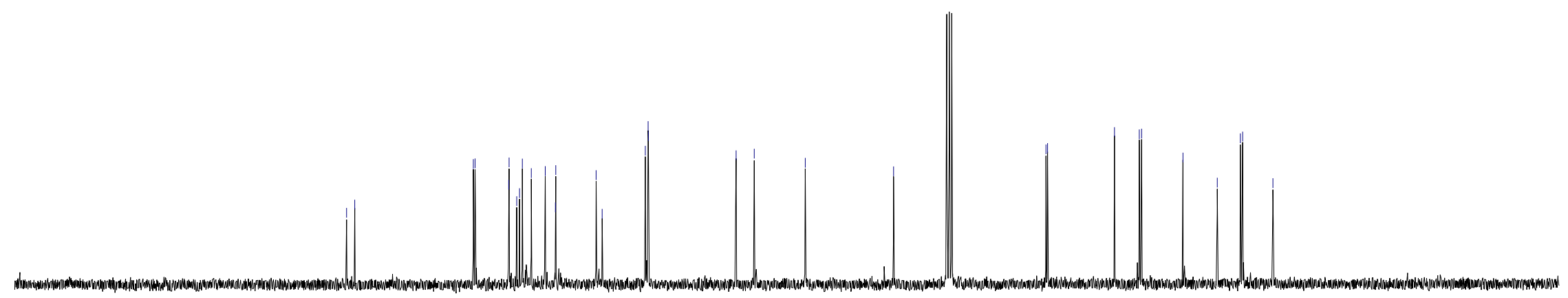




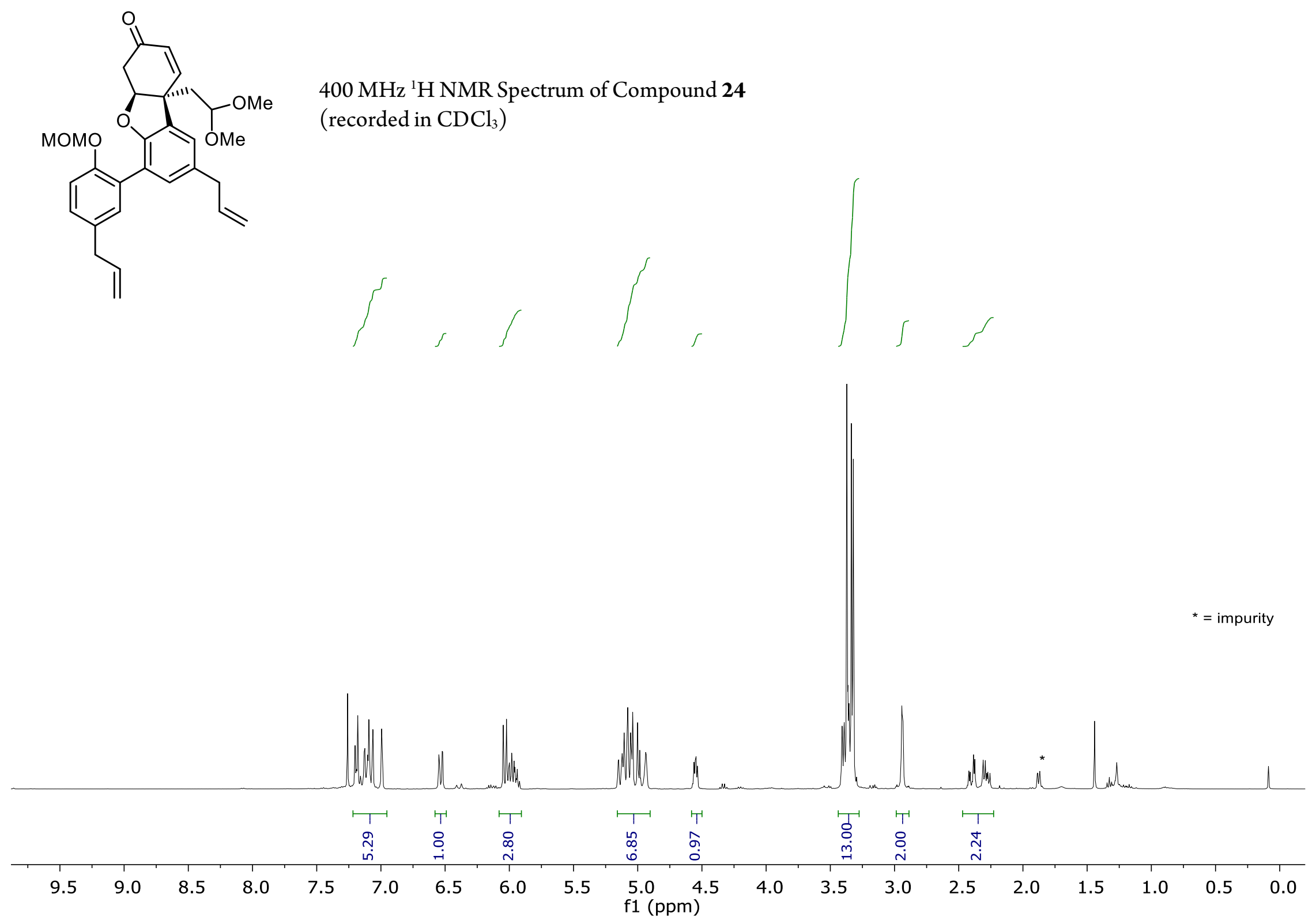



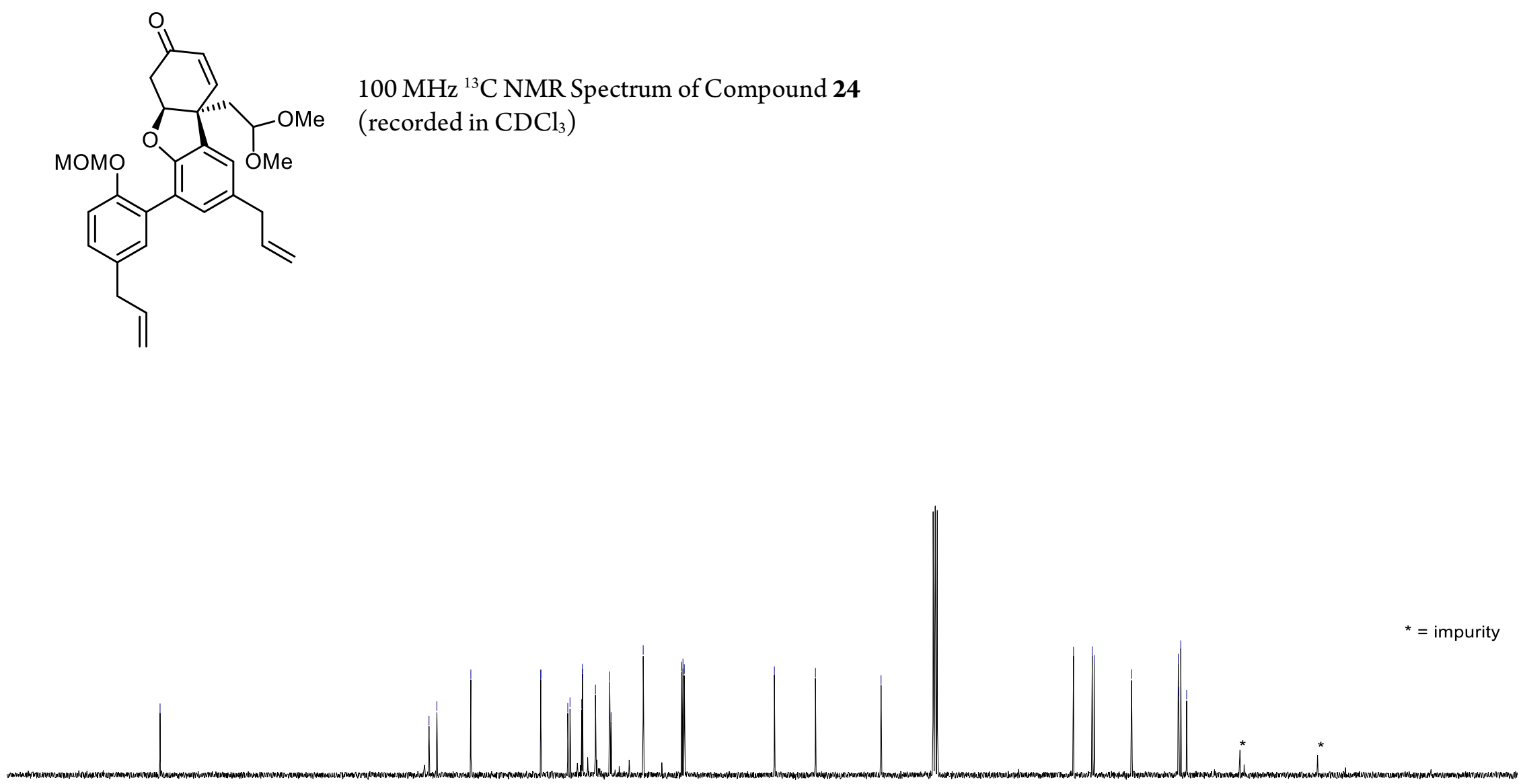


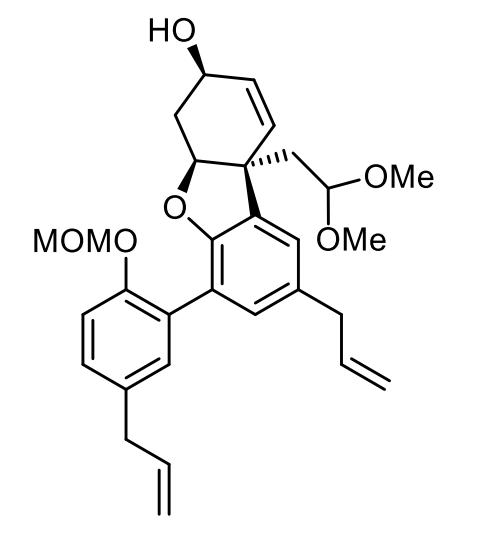

$400 \mathrm{MHz}{ }^{1} \mathrm{H}$ NMR Spectrum of Compound 25

(recorded in $\mathrm{CDCl}_{3}$ )

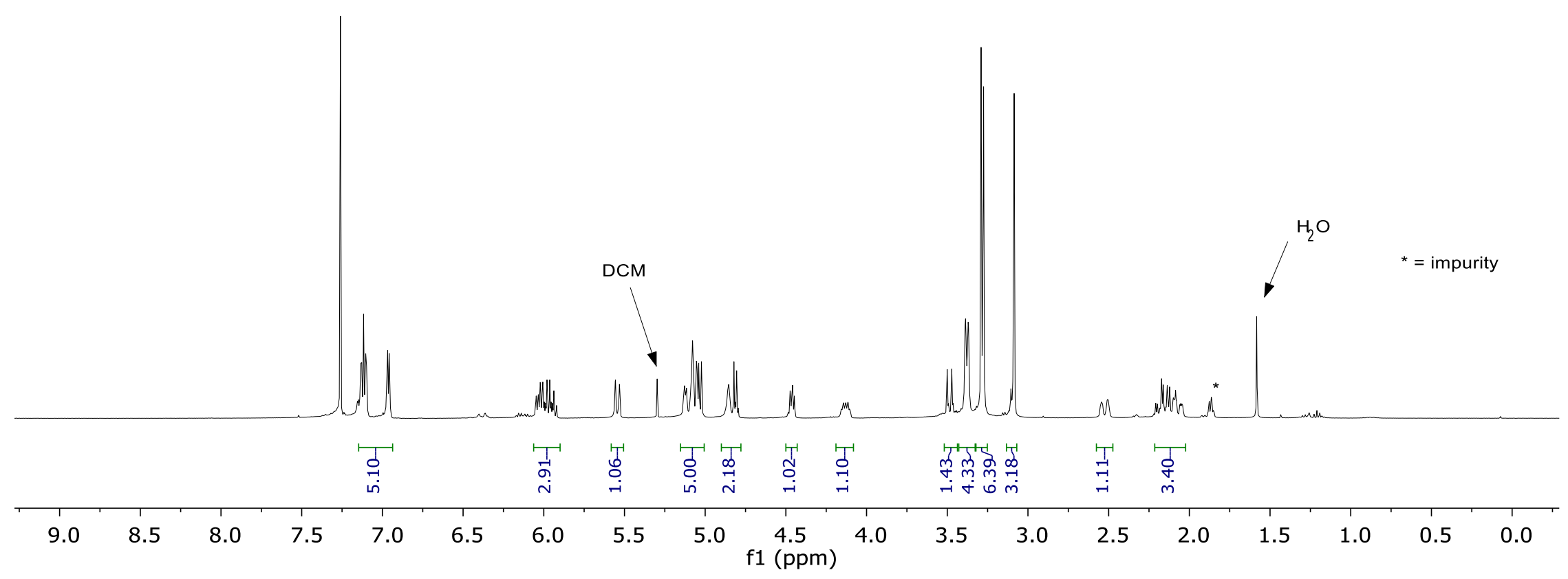




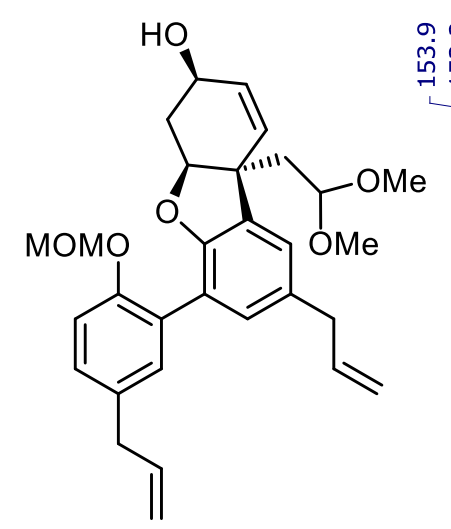

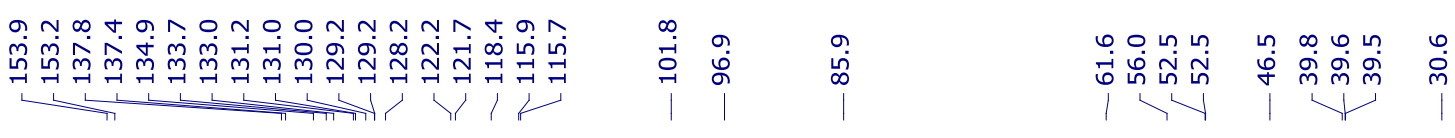

$100 \mathrm{MHz}{ }^{13} \mathrm{C}$ NMR Spectrum of Compound 25

(recorded in $\mathrm{CDCl}_{3}$ )

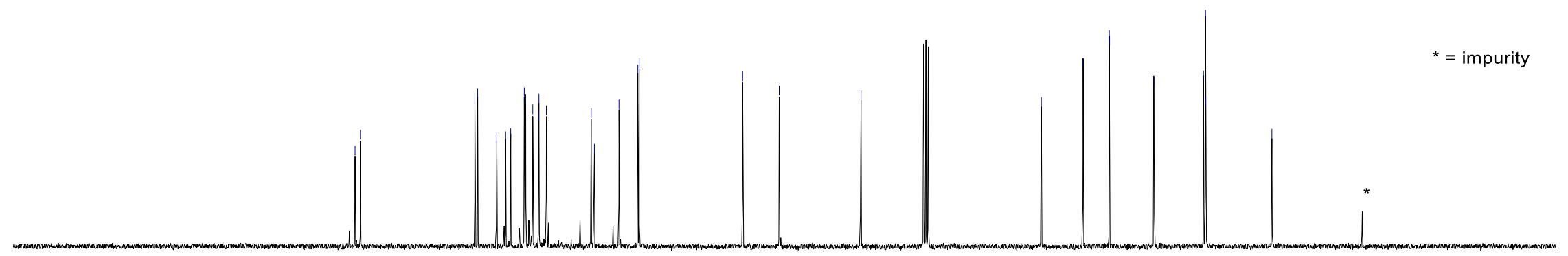

190 


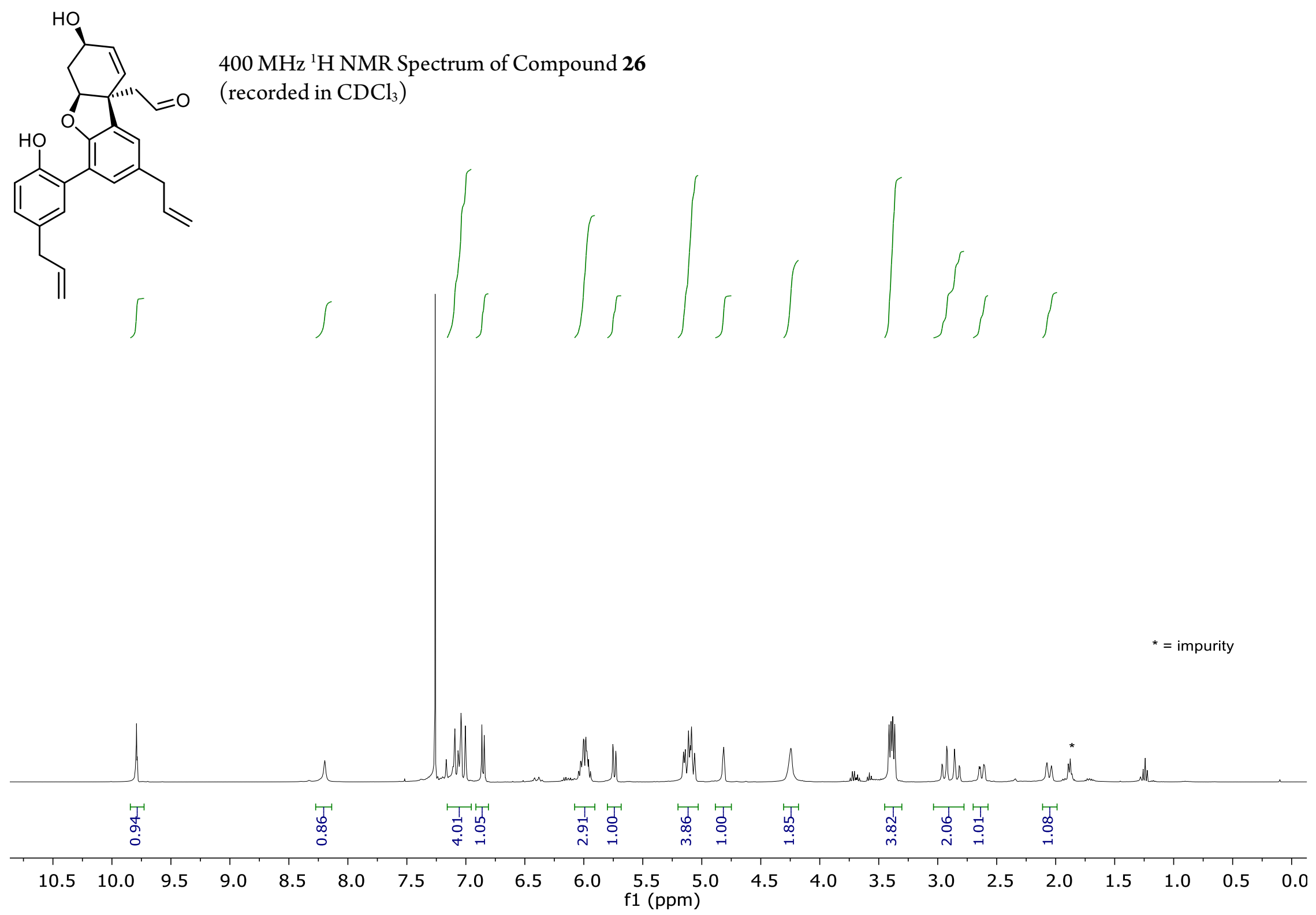




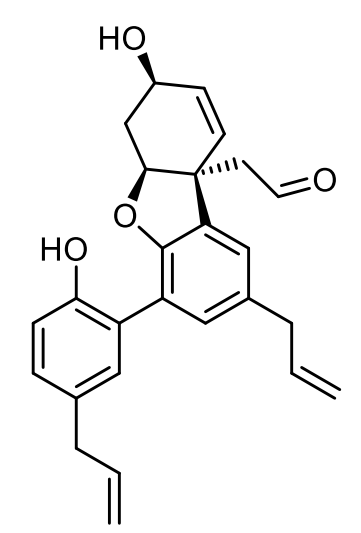

$100 \mathrm{MHz}{ }^{13} \mathrm{C}$ NMR Spectrum of Compound 26 (recorded in $\mathrm{CDCl}_{3}$ )

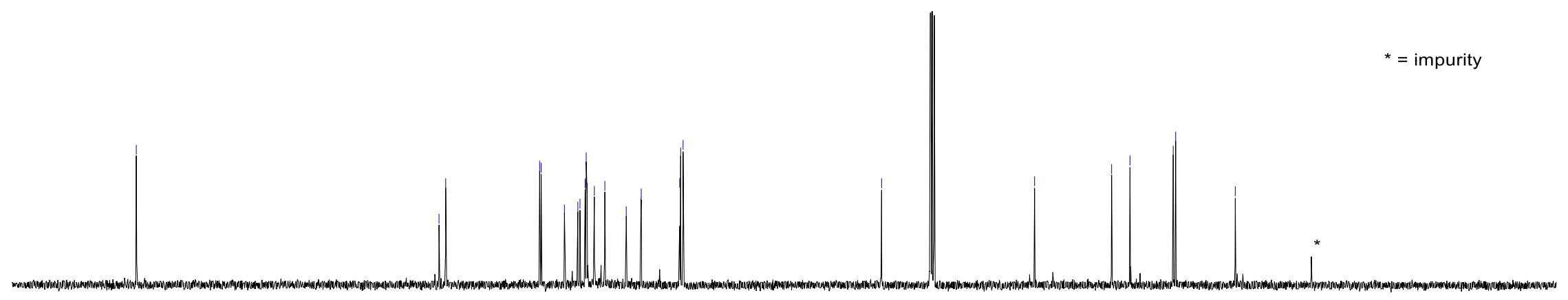




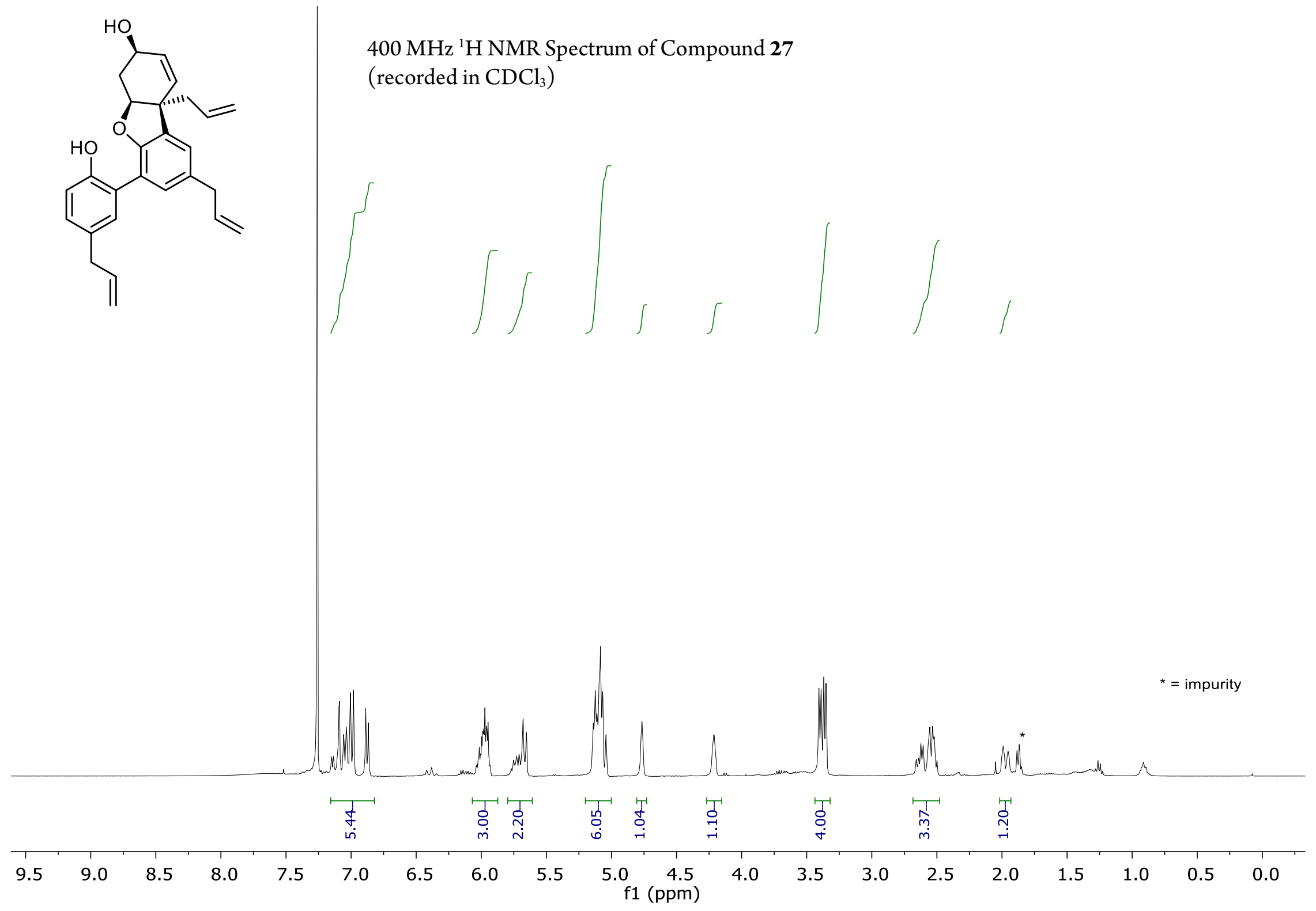



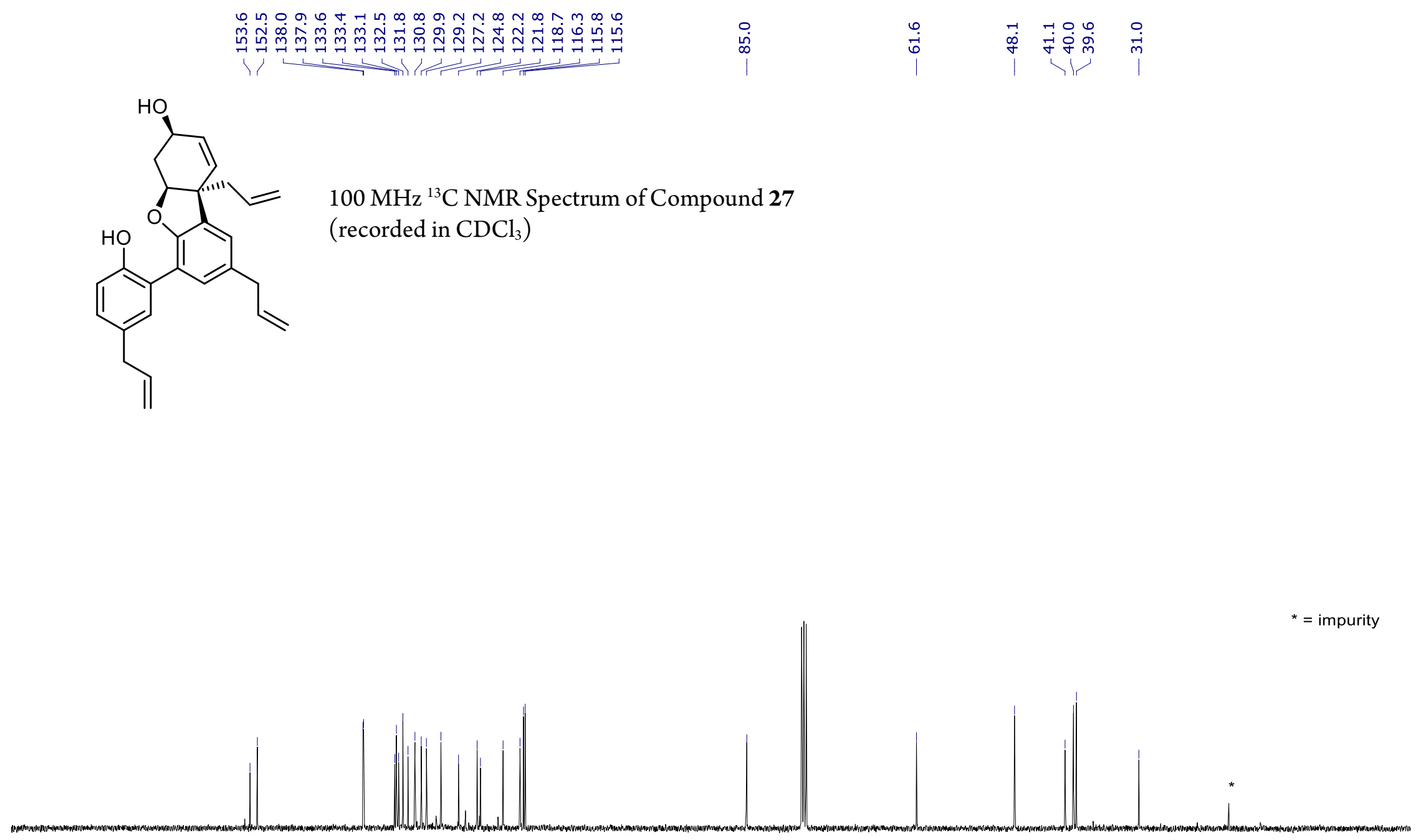


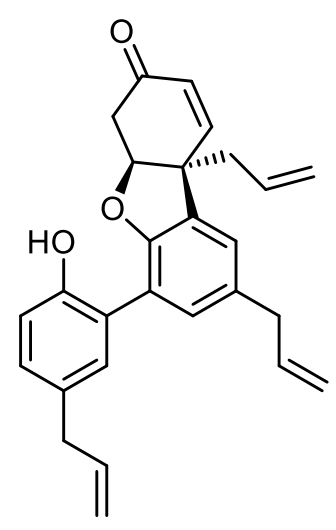

$400 \mathrm{MHz}{ }^{1} \mathrm{H}$ NMR Spectrum of Compound 1

[recorded in $\left(\mathrm{CD}_{3}\right)_{2} \mathrm{CO}$ ]
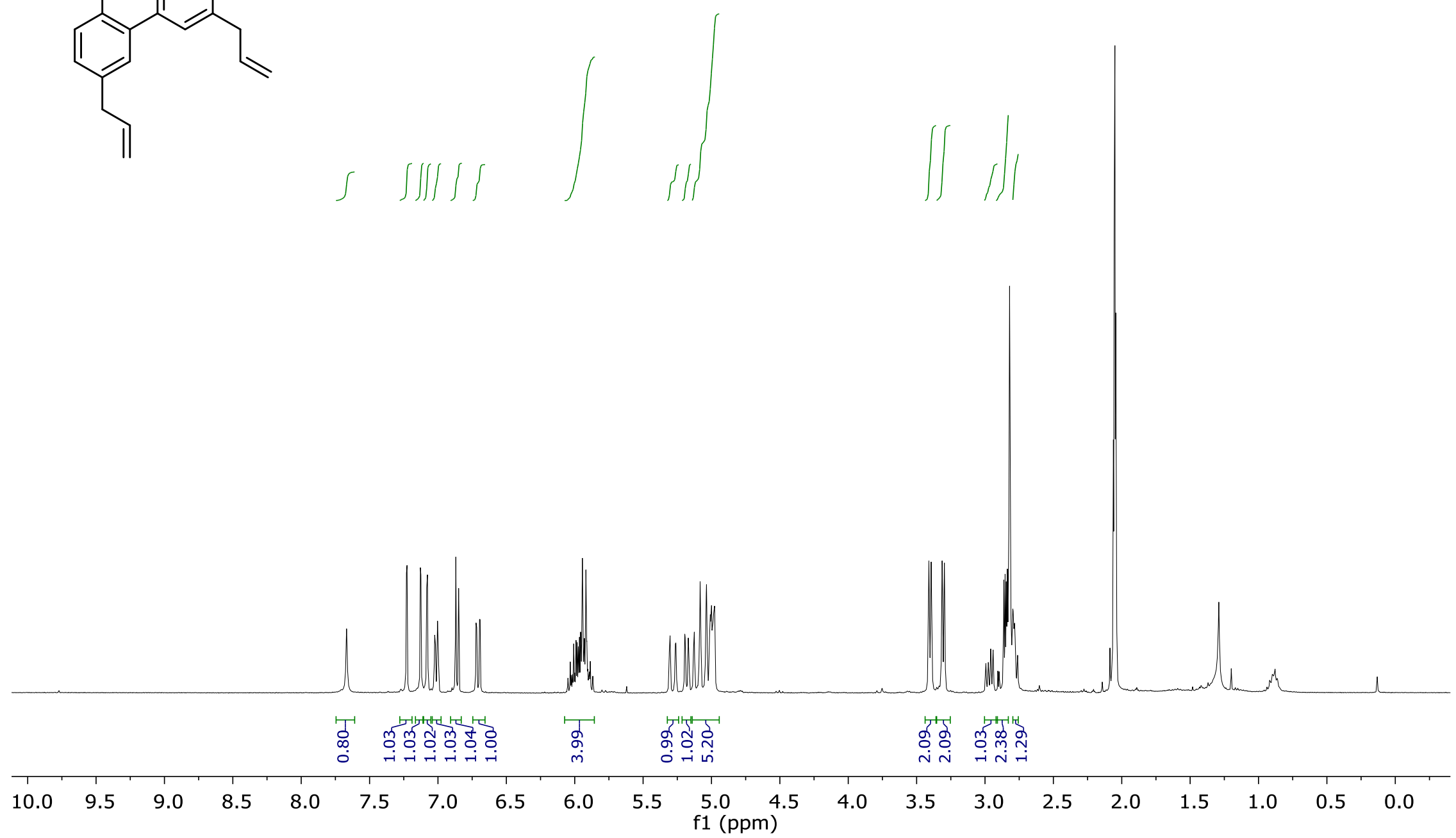


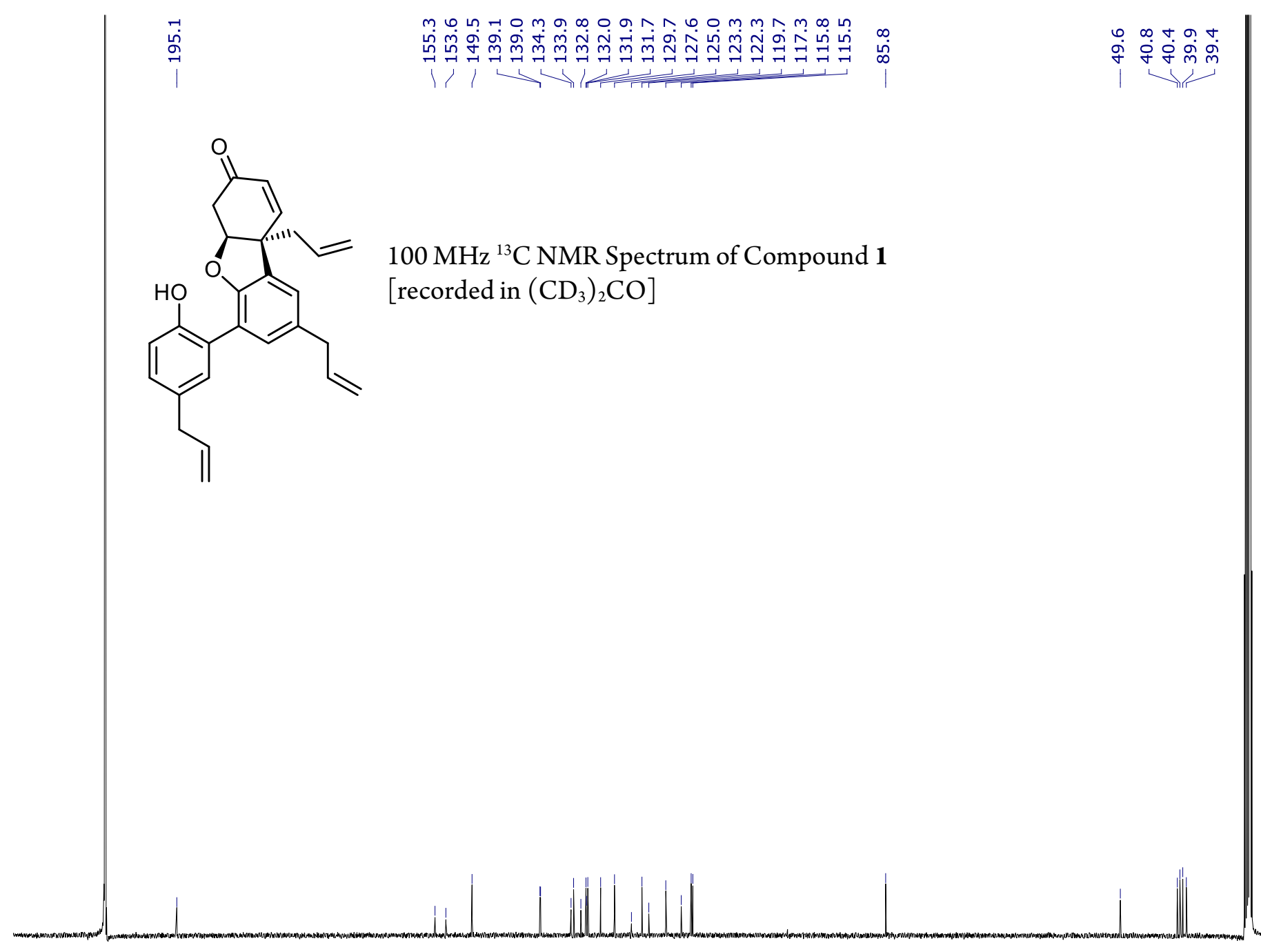

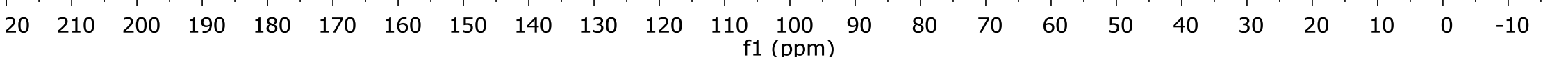

